NBSIR 86-3430

\title{
REVISED INTERIM DESIGN GUIDELINES FOR AUTOMATED OFFICES
}

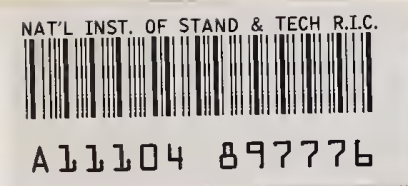

NIST

PUBLICATIONS

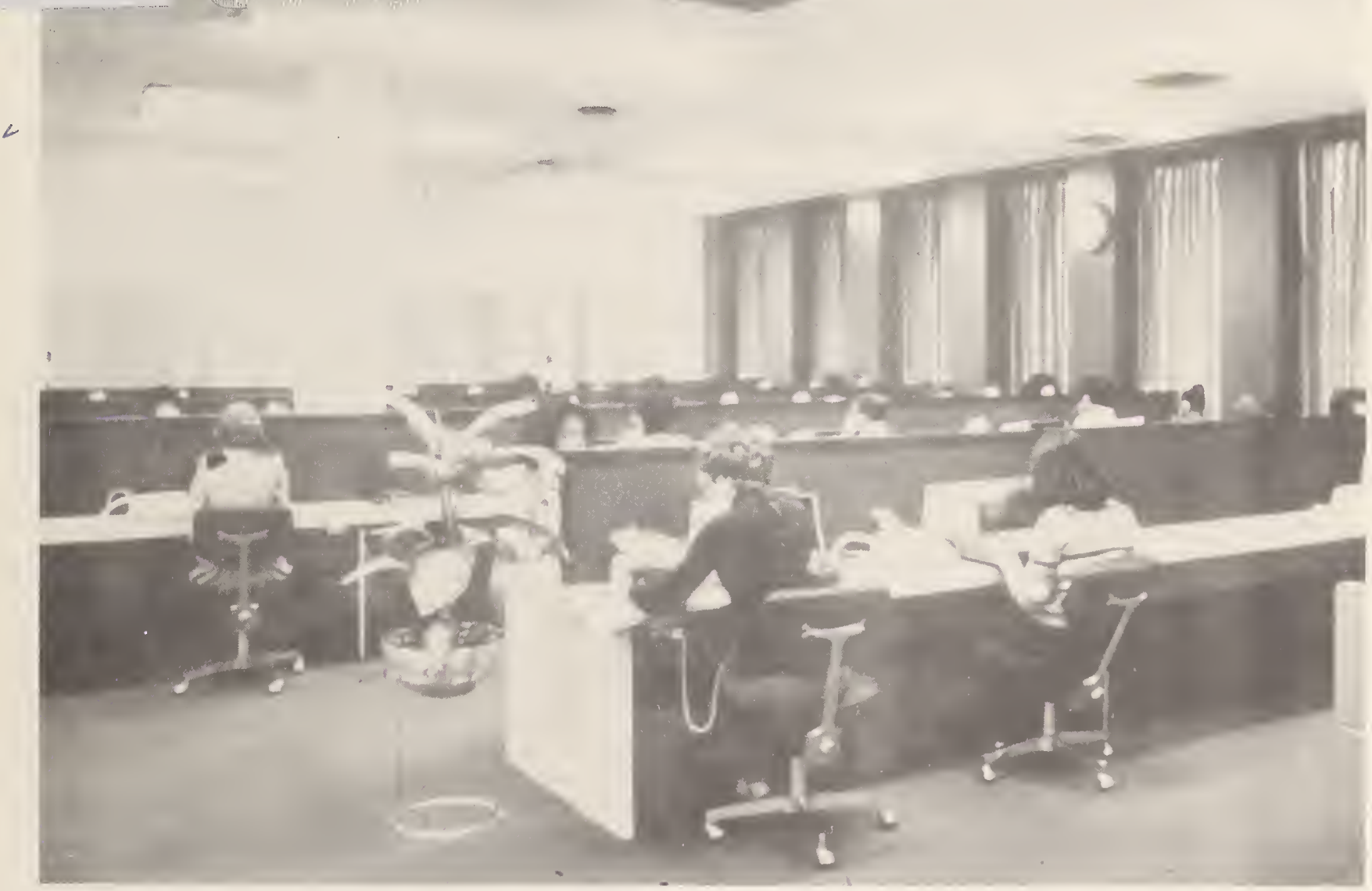

August 1986

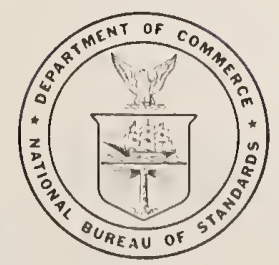

Center for Building Technology

QC

100

.056 NO. 863430 1986

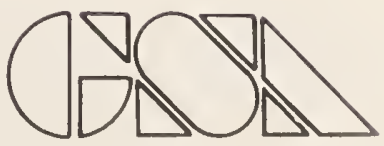

General Services Administration Washington, DC 20405 



\section{REVISED INTERIM DESIGN GUIDELINES FOR AUTOMATED OFFICES}

Arthur Rubin

U.S. DEPARTMENT OF COMMERCE National Bureau of Standards National Engineering Laboratory Center for Building Technology Building Physics Division Gaithersburg, MD 20899

August 1986

Prepared for:

General Services Administration

Washington, DC 20405

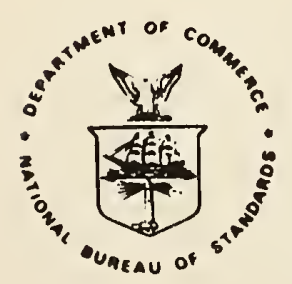

U.S. DEPARTMENT OF COMMERCE, Malcolm Baldrige, Secretary national buREaU OF STANDARDS, Ernest Ambler, Director 
I would like to express my appreciation to $\mathrm{Mr}$. John J. Landers of the Office of Information Resource Management of the General Services Administration for his support and advice during the course of the project. I would also like to thank the following reviewers for their constructive comments and critiques of the report: Herbert Rosenheck, Lisa Sauerwein, Vivian Loftness, Dr. Forrest Wilson, Dr. Belinda Collins, Dr. Tamami Kusuda, and Roger Rensberger. 
This report presents an update of the first edition of "Interim Design Guidelines for Automated Offices" , August 1984 (NBSIR 84-2908). It is based upon findings obtained by an extensive literature search, a questionnaire survey of design firms, an office automation roundtable, and interviews with design and other office automation professionals. The guidelines should still be considered tentative, since the information is based primarily on judgment, rather than on formal investigations or on broad consensus from the design community. Furthermore, many important design issues have not been dealt with, because they have not yet been appropriately considered,and/or it is unclear what the design implications of automation are - e.g. will overall space needs be greater or less?

The introduction of automated systems into offices has changed the office setting as a place to work. Architects and other design professionals have responded to this technology by formulating a variety of design strategies. This report identifies design issues which merit consideration in automated offices, tentative criteria for environments and systems based on an overview of all resources used to develop this document, and typical approaches employed to accomplish design goals. Technological, ergonomic and organizational factors are considered from the standpoint of their design implications. This updated report is a major revision of the earlier work, including more than twice the number of reference documents as the initial study.

Rey words: acoustics; design criteria; electrical systems; ergonomics; lighting; noise; office automation; office design criteria; office furnishings; safety systems; workstation; communication systems; LAN's; data centers. 


\section{PREFACE}

This report is the fourth of a report series prepared by NBS as part of Memoranda of Understanding and Agreement between NBS and the GSA, dated $1 / 16 / 81$ and 2/19/85. This work examines the impact of office automation technologies on interior environments, and provides guidance for federal office design. The earlier publications, sponsored by the Public Building service of GSA are as follows:

1. The Automated Office - An Environment for Productive Work, or an Information Factory?: A Report on the State-of-the-Art, NBSIR 832784-1, November 1983.

2. The Automated Office - An Environment for Productive Work, or an Information Factory?: Executive Summary, NBSIR 83-2784-2, December 1983.

3. Interim Design Guidelines for Automated Offices, NBSIR 84-2908, August 1984 .

This revision, sponsored by the Information Resources Management Service of GSA, updates the earlier work by a more extensive treatment of communincation and informational issues; e.g. communication systems, intelligent buildings, and data processing center design. It is intended to assist in developing functional, economical and humane environments for federal office buildings. It provides design information for those charged with the control, administration and maintenance of office environments with varying degrees of automation.

The document is a compilation and evaluation of design practices, approaches, tentative criteria, and design factors to be considered in meeting individual and organizational needs. The information available is limited and not definitive, since few formal studies have dealt with this subject and little consensus exists among architects regarding appropriate practices. Some basic assumptions were made in preparing this report:

*Office automation is a continuing process, not a discrete event. *The office environment will consist of electronic and paperbased tasks for the indefinite future. *Office work will continue to be performed in spaces and buildings designed for this purpose. *The "typical office" is as meaningless a concept as the "average" worker, and the "prototype" organization.

* Energy conservation will be of continuing importance. * Consideration of long-term costs are more important than first costs.

*Office design influences worker productivity; high quality environments are essential for automated offices. * Better information is needed to formulate performance criteria for automated offices. 
1. Int roduction $\ldots \ldots \ldots \ldots \ldots \ldots \ldots \ldots \ldots \ldots \ldots \ldots \ldots$

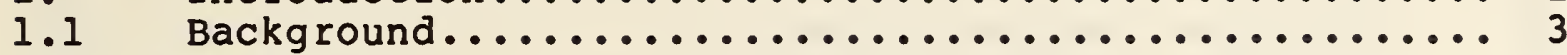

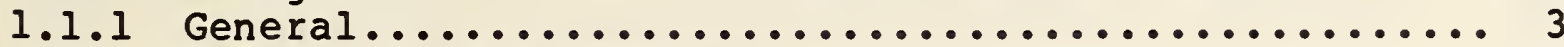

1.1 .2 Technology and Design $\ldots \ldots \ldots \ldots \ldots \ldots \ldots \ldots \ldots \ldots \ldots$

1.1 .3 Organizations and Design ..................... 7

1.1 .4 Ergonomics and Design ........................ 8

1.1 .5 Security and Design $\ldots \ldots \ldots \ldots \ldots \ldots \ldots \ldots \ldots \ldots$. 8

1.1 .6 Planning $\ldots \ldots \ldots \ldots \ldots \ldots \ldots \ldots \ldots \ldots \ldots \ldots \ldots \ldots \ldots$

2. Planning and Programming the Automated office...... 10

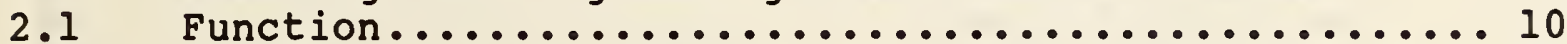

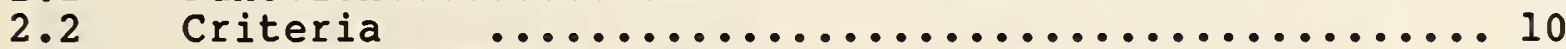

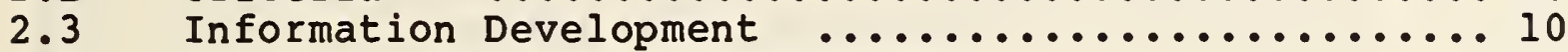

2.4 Planning Approaches- Programming .............. 13

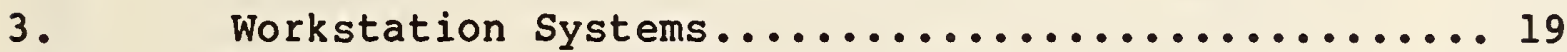

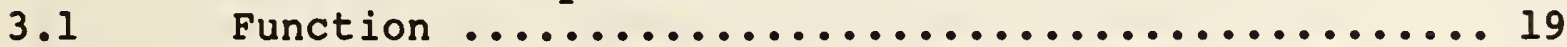

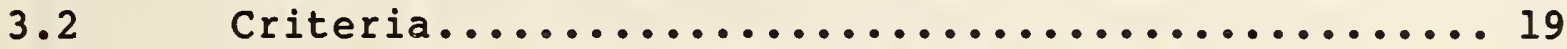

3.3 Furnishings- Flexibility Requirements ......... 23

3.3.1 Checklist -- Workstation Flexibility ........... 23

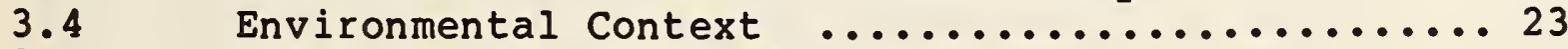

3.4.1 Checklist for Workstations

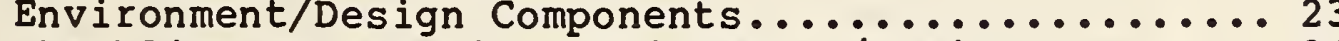

Checklist Workstation Environment/Design .......24

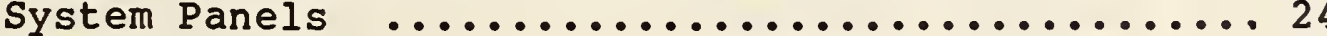

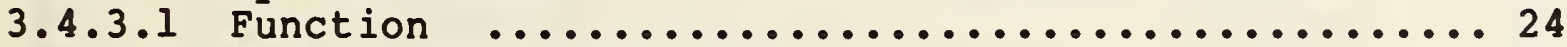

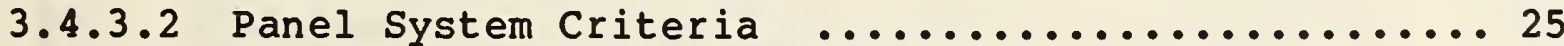

3.4.3.3 Checklist for Panel Components .............. 26

3.4.3.4. Interactions with other systems $\ldots \ldots \ldots \ldots \ldots \ldots 28$

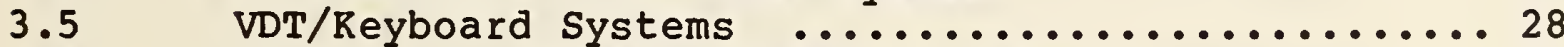

3.5.1 Checklist- VDT/Reyboard Requirements ........... 29

3.6 Possible Problem Area - VDT Screen Glare ......... 29

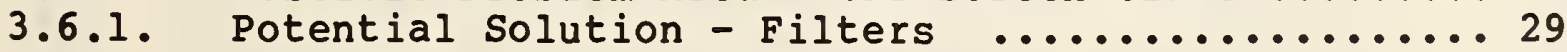

4. Acoustic Environmental Systems .............. 31

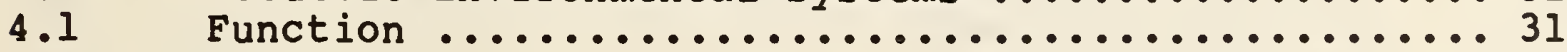

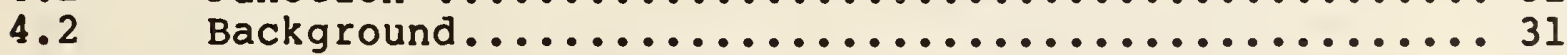

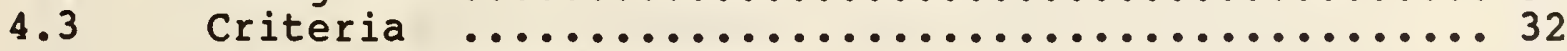

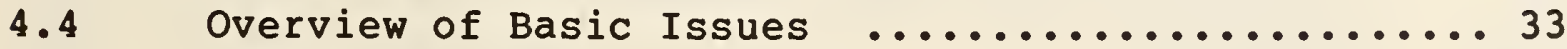

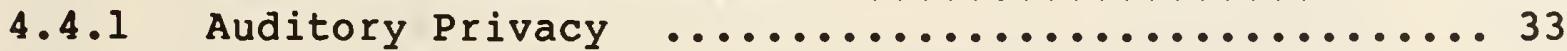

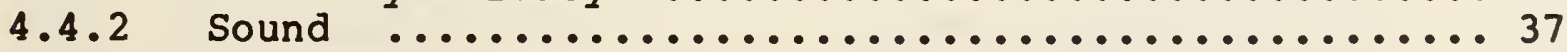

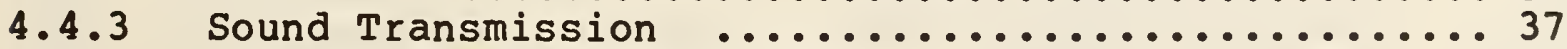

4.4.4 Noise Criteria (NC) Curves ...........................

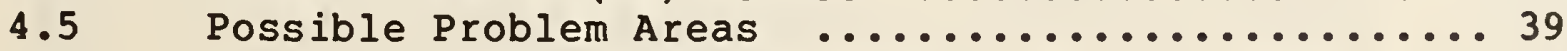

4.6 Typical Solutions $\ldots \ldots \ldots \ldots \ldots \ldots \ldots \ldots \ldots \ldots \ldots \ldots \ldots . \ldots \ldots$

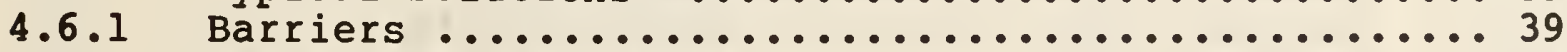

4.6 .2 Isolation of Surfaces $\ldots \ldots \ldots \ldots \ldots \ldots \ldots \ldots \ldots \ldots \ldots . \ldots . \ldots . \ldots$

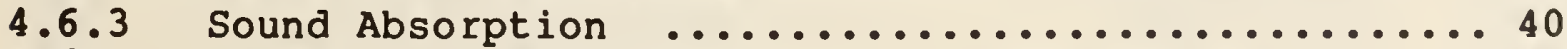

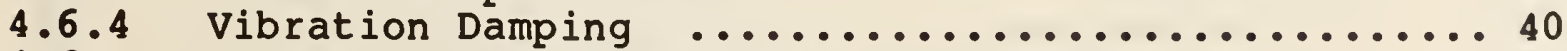

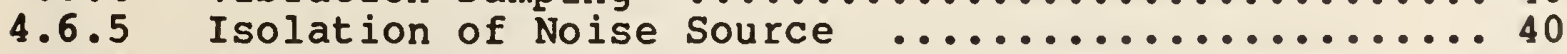

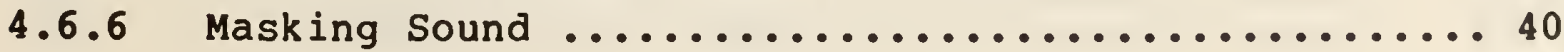


4.6.7 Ceilings

4.6.8 Distance

Configuration

Floors

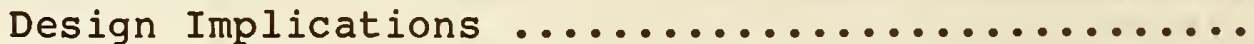

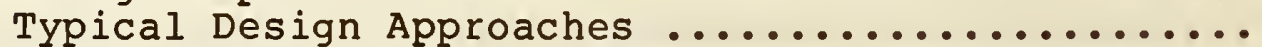
Common Sources of Static Electricity ........... Possible Approaches to Overcome Problem ......... Air Quality 
Electrical Power Systems

Function

Background

Criteria

\section{Flat}

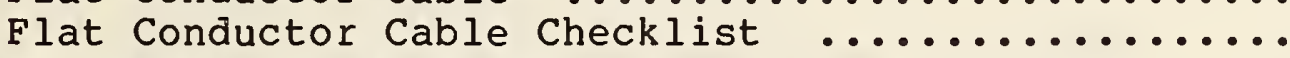

Tradeoffs among Alternative Systems .......... 92 Additional Design Considerations ............ 94

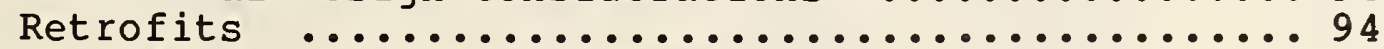
Upgrading of Cabling Systems ................ 94 Environmental Context $\ldots \ldots \ldots \ldots \ldots \ldots \ldots \ldots \ldots . \ldots \ldots$ Checklist-Wiring System Design ............. 94

9 .

9.1

9.2

9.3

9.3 .1

9.3 .2

9.4

9.4 .1

9.4 .2

9.5

9.5 .1

9.5 .2

9.6

9.6 .1
Communication Systems $\ldots \ldots \ldots \ldots \ldots \ldots \ldots \ldots$

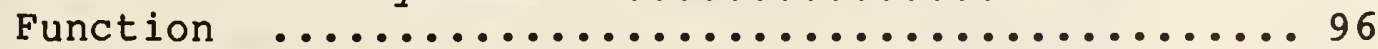

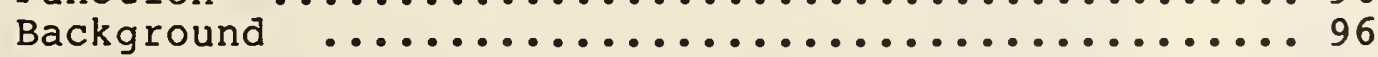

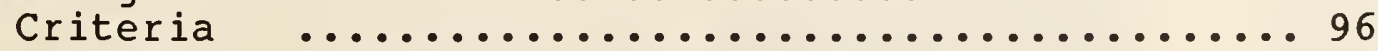

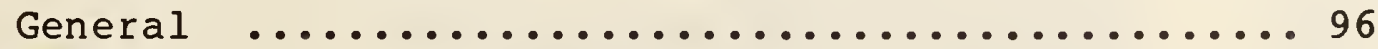

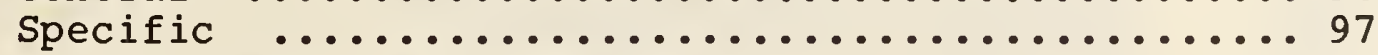

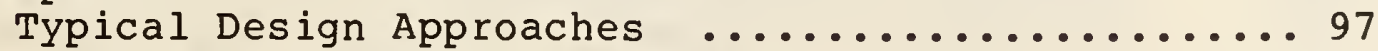

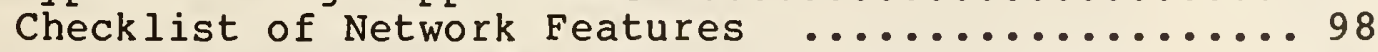
Checklist - Design for Communications Systems ....100

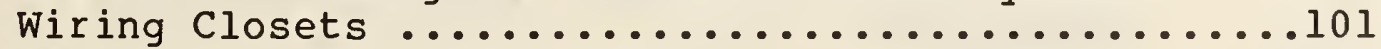

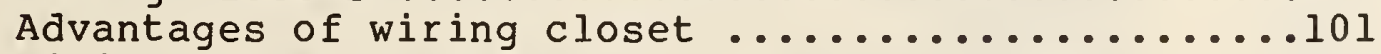

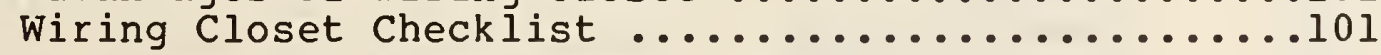
Telecommunications Accessories ................102 Functions 


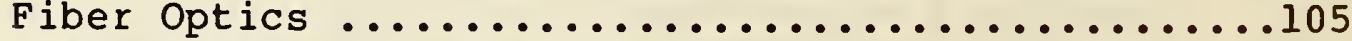

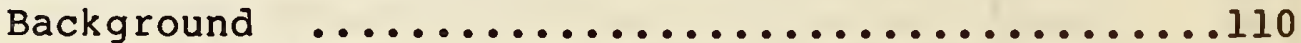

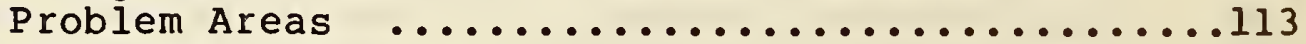

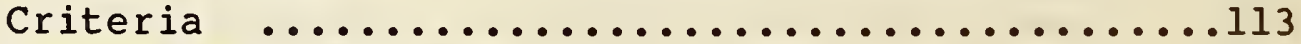

10.4

10.5

10.6

10.7

10.7 .1

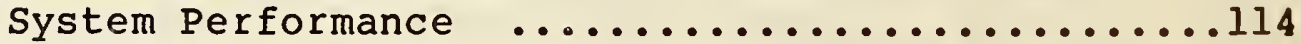

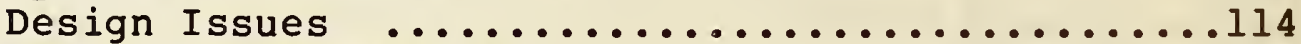

Technological Issues $\ldots \ldots \ldots \ldots \ldots \ldots \ldots \ldots \ldots \ldots \ldots$

Organizational Issues $\ldots \ldots \ldots \ldots \ldots \ldots \ldots \ldots \ldots \ldots$

Software Issues $\ldots \ldots \ldots \ldots \ldots \ldots \ldots \ldots \ldots \ldots \ldots \ldots \ldots \ldots$

Typical Design Approaches ..................118

10.7 .1 .1

10.7 .1 .2

Network Configurations $\ldots \ldots \ldots \ldots \ldots \ldots \ldots \ldots \ldots \ldots$

10.7 .1 .3

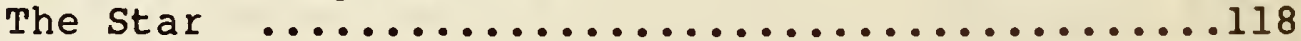

10.7 .1 .4

10.8

10.8 .1

10.8 .2

10.8 .3

The Ring

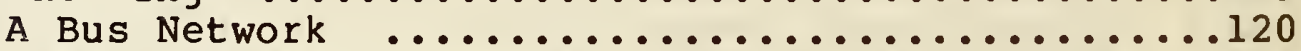

Tradeoffs Among Alternative LAN Configurations ..121

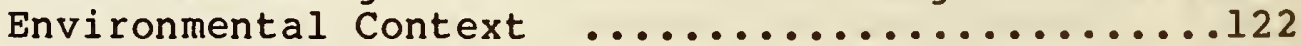

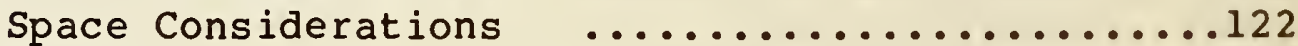

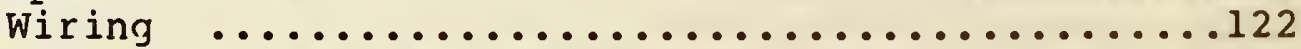

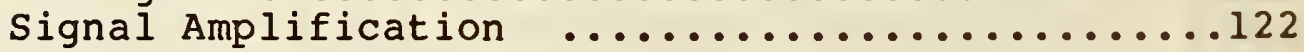

11

11.1

11.2

11.3

11.4

11.4 .1

11.4 .2

11.5

\section{PBXS}

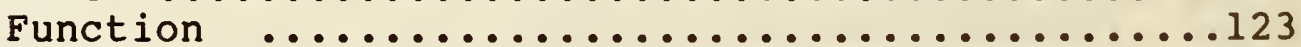

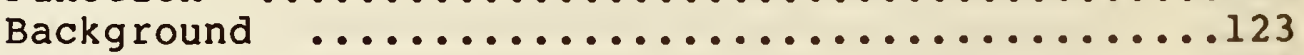

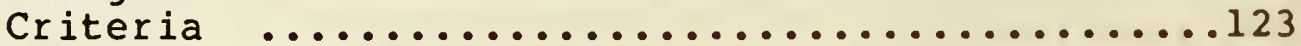

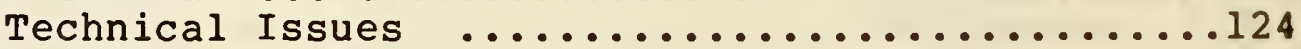

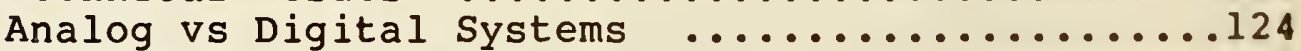

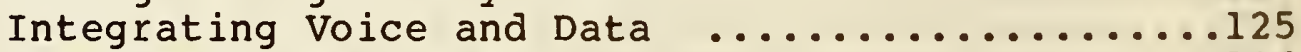

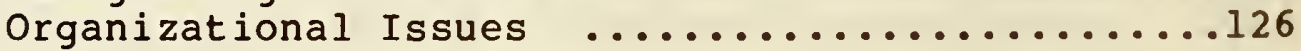

12 .

12.1

12.2

12.3

12.4

12.5

12.6

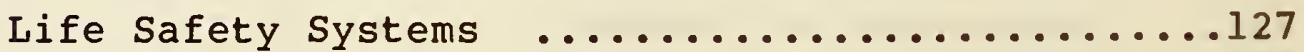

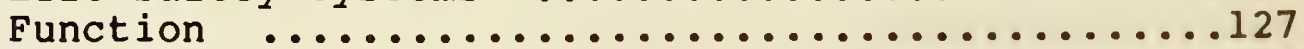

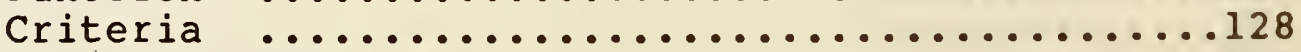

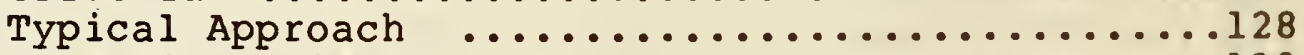

Checklist-Life Safety Systems ............130

Checklist-Central Control ................130

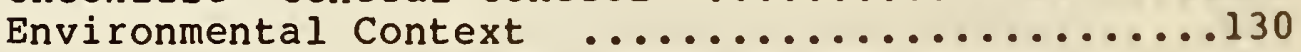

Intelligent Buildings- Integration Issues ....132

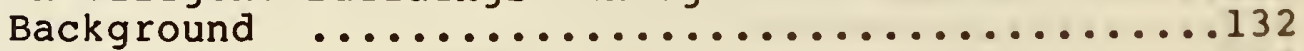

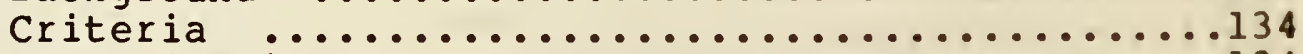

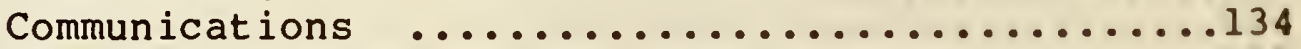

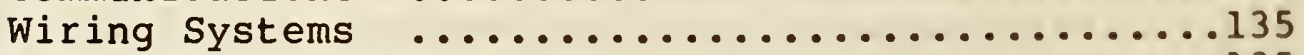

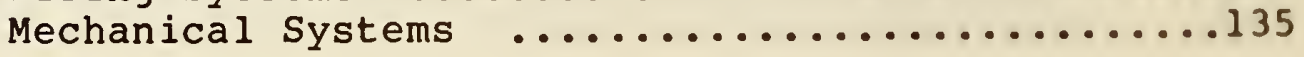




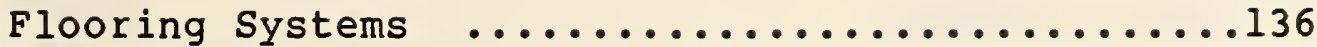

15.2

Overview

15.2 .1

Teleconf

15.2 .2

Function

15.2 .3

Criteria

encilities

Environmen

................

15.2 .4

15.2 .4 .1

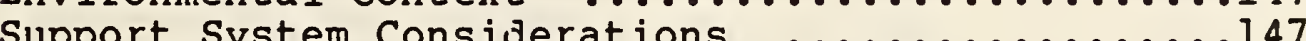

15.2 .4 .2

Acoustics

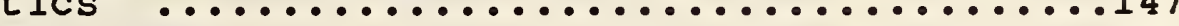

$15.2 \cdot 4.3$

HVAC Equipment

15.3 .

15.3 .1

Lighting and Color

Data Center

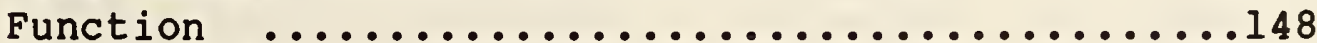

15.3 .2

$15 \cdot 3 \cdot 3$

15.3 .4

$15 \cdot 3 \cdot 5$

Background

[....................

148

Criteria for Computer Room Design ..........149

Air Conditioning

Electrical Power

$15 \cdot 3 \cdot 5 \cdot 1$

Electrical Power Checklist

15.3 .6

15.3 .7

Fire Detection and Suppression $\ldots \ldots \ldots \ldots \ldots \ldots 1$

15.3 .8

Security

Raised Access Floors

15.3 .9

Lighting

16 . 
Table 1 . Table 2 .

Table 3 .

Table 4. Table 5 .

Table 6 . Table 7 .

Table 8 . Table 9 .

Table 10.

Table 11. Table 12. Table 13. Table 14. Table 15.

Table 16. Table 17. Table 18. Table 19. Table 20. Table 21 . Table 22. Table 23. Table 24. Table 25.

Table 26. Table 27.

Table 28.
Environment as Support and Catalyst ........ 4 Checklist - Design Considerations for ${ }^{\circ}$ High Quality' Offices (Criteria) ............ 8 Analytic Approach for Relating Technology to

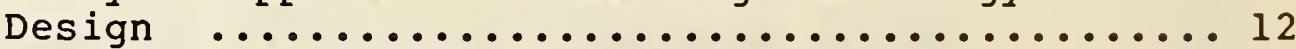
Checklist for Office Automation Design ....... 13 Introduction of Automation into Offices-

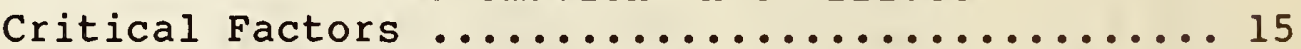
Recommended Workstation Dimensions for VDT's .... 20 Minimum federal performance requirements for

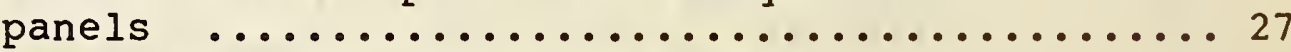

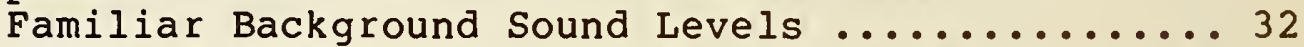
Types of Offices Applicable to Articulation Index Ranges Speech Interference Levels (SIL) and Noise

Criteria (NC) Recommended for Rooms ........... 34 Conversations and Speech Interference Levels .... 36 Lighting Recommendations (IES) ........... 47 Lamp Selection Guide ..................... 51

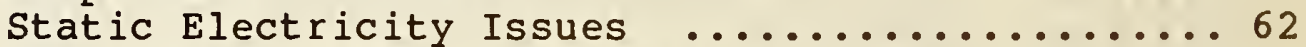
Basic Cause, Effect and Treatment of Power Line

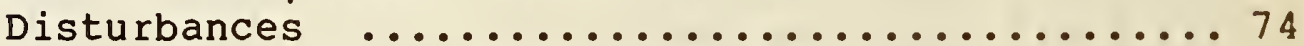
UPS Guide to Installation and Maintenance ..... 76 Comparison of Power Protection Systems .......7 78 Cable Characteristics .....................107 Wiring Media Comparisons ..................108 Comparison of LAN and PBX features ...........109 Cost Proportions of LAN's ..................112 LAN Market Share by Type .................112 Requirements for a LAN .................113 Typical Throughput Applications for LAN .......115 Positive and Negative Features of LAN

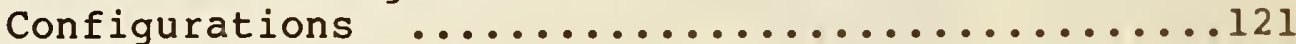

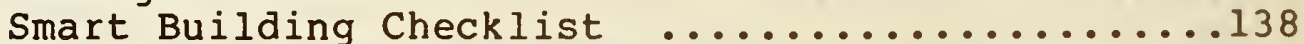
Integration and Telecommunication systems -

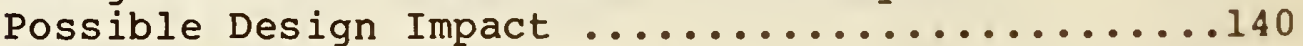
Carpet Materials - Positive and Negative

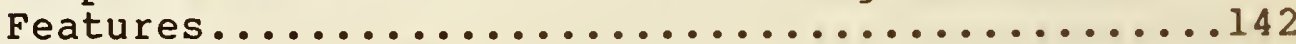


Figure 1. Environment as support and catalyst ......... I

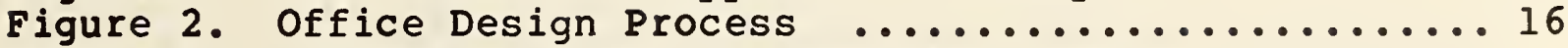
Figure 3. Conceptual Model for Evaluating Work

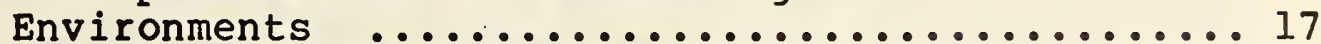

Figure 4. Checklist for Facility Evaluation ........... 18

Figure 5. Suggested Parameters for Mockup of a Seated

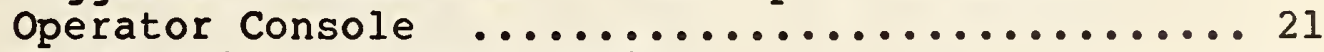

Figure 6. Workstation and Seat Design .............. 22

Figure 7. Approximate relation between AI and Speech

Intelligibility $\ldots \ldots \ldots \ldots \ldots \ldots \ldots \ldots \ldots \ldots \ldots \ldots \ldots$

Figure 8. Noise Criteria (NC) Curves ............... 38

Figure 9. Simplified block diagram of OPLAN program ..... 42

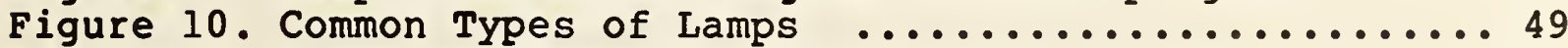

Figure 11. Types of Illumination $\ldots \ldots \ldots \ldots \ldots \ldots \ldots \ldots \ldots$

Figure 12. Thermal Comfort Recommendations (ASHRAE) ...... 58

Figure 13. Conventional ceiling air distribution ........ 59

Figure 14. Poke-Through System ................. 85

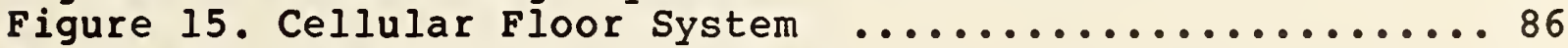

Figure 16. Underfloor Duct System ................ 87

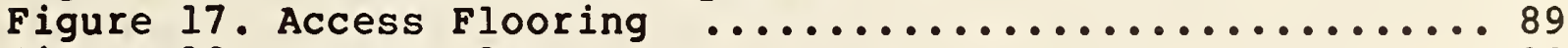

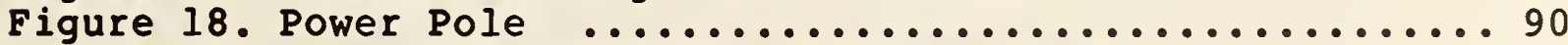

Figure 19. Network Applications and Features .......... 98

Figure 20. Star network topology ..................118

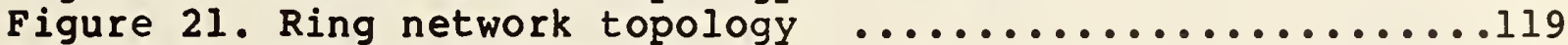

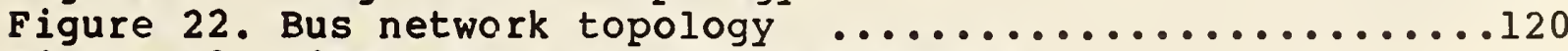

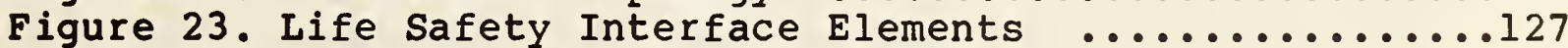

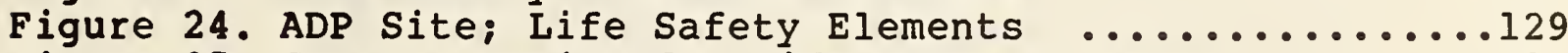

Figure 25. Control Design for Life safety system .........131

Figure 26. Typical static voltages generated by walking

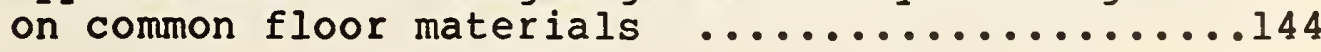





\section{INTRODUCTION}

Office design has a major role to play in the work performance of the incividual, and thereby in the functioning of an organization as a whole. While the physical setting of an office provides the direct support for conducting activities in a space, the environment also serves a catalytic function. It can promote or inhibit discussions, meetings, and formal or informal

interactions among people. Space characteristics, lighting, temperature levels, humidity, indoor air quality and other design features can have positive or negative influences on task performance and/or satisfaction of the worker (1).

Questionnaire surveys consistently have shown the work environment is an important contributor to satisfaction and morale of office workers, thereby influencing productivity. The automation of office functions has further increased its significance for several reasons.

The characteristics of the office workforce are rapidly changing, as is the nature of the jobs being performed. Technology has influenced the way that organizations are structured, and how decisions are made. The fastest growing segment of the office population, and the key to increased productivity are knowledge workers- (professionals and managers) who differ in many respects from their clerical counterparts. Knowledge workers are highly educated, have experienced high quality work environments and expect such settings. They also do not hesitate to express dissatisfaction with environmental distractions and intrusions on their privacy. Since their skills are readily transferable from one organization to another, they are a highly mobile group. Under these circumstances, the work setting is important in recruiting and retaining qualified people, because other aspects of their jobs are often comparable in terms of salary and other features (2). (See Figure l.)

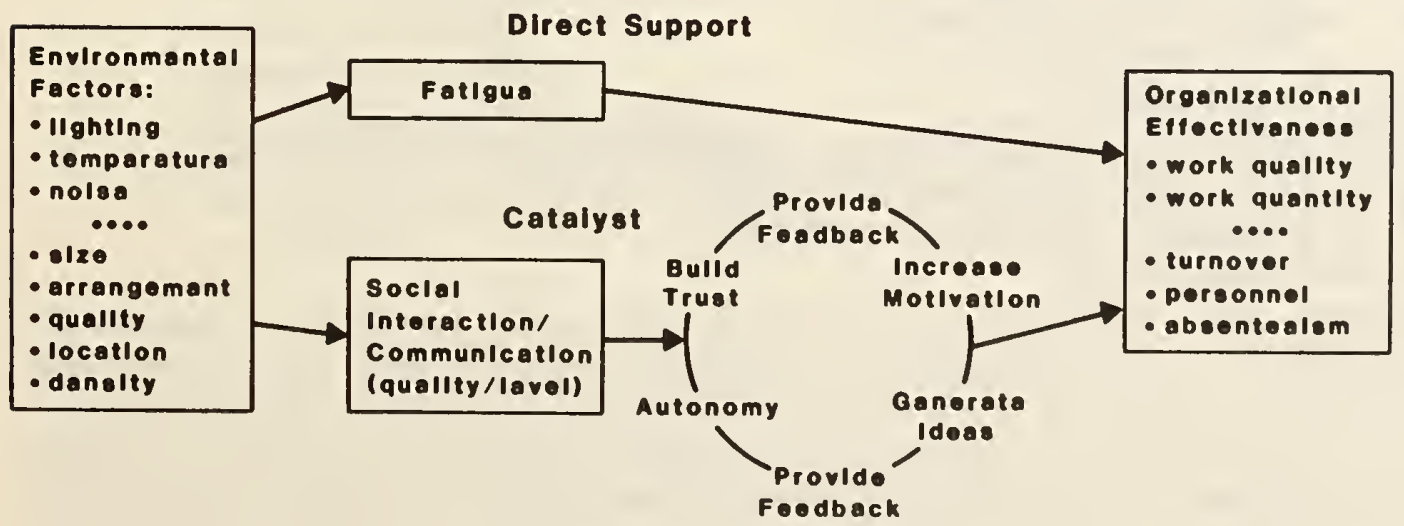

Figure 1. Environment as support and catalyst 
The work performed by knowledge workers using automated equipment is mentally demanding and requires freedom from distractions such as glare, noise, and the activities of their colleagues. The tasks are often intrinsically difficult, as in the case of planning and problem solving. Personal interactions, which characterize many management functions, require auditory and visual privacy. The process required to perform VDT-based activities imposes a further burden on employees with limited training and/or skills in using keyboard devices or the specialized programming languages required to communicate with office automation devices such as microprocessors. They are also frequently required to access external sources such as information networks. Employees, often working at VDT terminals for eight hours a day, are quite sensitized to their surrounds, and require a respite from high technology, and an opportunity for social contact in pleasant settings.

Management style is an important determinant of the overall appearance and layout of the traditional office and particularly the automated one. Decisions concerning the balance between design uniformity and diversity, within an organization as a whole, among working groups, for the management hierarchy, and for individuals, all reflect the organizational culture. A centralized management approach is characterized by considerable uniformity and little room for personal expression by the individual. Decentralization provides the opportunities for working units and individuals to participate in the design of their environments, and encourage differentiation among groups and individuals. In this way, a sense of group cohesiveness can be expressed, as well as a means of countering the institutionalization of the office. Specialized spaces such as breakout conference rooms are needed to enhance communication in this way. In many instances, tradeoffs must be made between uniformity and the possibility of expressing diversity.

A key problem identified in surveys of workers in automated offices is that of work stress. Among its potential causes are:

* Rigid work procedures

* Oversimplified, repetitive and routine work

* Lost sense of job meaning

* Lack of control

* Heavy workloads

* Pressures for performance; monitoring of performance

* Disrupted social relationships

* Reduced status and self-esteem

* Reduced mobility

* Concern for safety

* Concern for job future 


\subsection{BACKGROUND}

1.1.1. General

Productivity in the office has been virtually stagnant for almost a decade, at a time when white collar work has begun to dominate our economy. For example, by 1983, 53 percent of the workforce was engaged in information development and processing activities. The trend toward more office-based activities, the productivity figures, the information explosion, technological advances accompanied by decreased costs, have all combined to create an intense demand to automate office functions. As a result, millions of new devices and systems are now in offices, and countless others are being introduced daily at an ever increasing rate. Yet the experience with office automation has been mixed.(3)

While technology is seen by some observers as THE answer to office productivity problems, considerable evidence exists that many office workers are less than satisfied with the quality of the environment in the automated office.

Too often, office automation has resulted from the piecemeal acquisition of new devices and systems placed on available desks and other work surfaces in environments designed to process information in paper form. VDT's have been introduced into the workplace without regard to the differences between them and paper-based tasks.

A comparison of VDT and paper-based tasks reveals important differences between the two:

\section{PAPER/HARDCOPY}

* Dark characters on a light background

* Continuous line characters

* Matte surface

* Horizontal plane

* Manual input

* Easy to handle

* Perceptually permanent

* Data stored physically

* Information presented serially

\section{ELECTRONIC/VDT}

* Light characters on a dark background

* Dot-matrix characters

* Reflective surface

* Vertical plane

* Reyboard input

* Difficult to manipulate

* Perceptually transient

* Electronic data storage

* Information presented simultaneously

Systematic design planning has been a rarity. Computer enhancements have been handled as retrofits for furniture acquisition. This process has resulted in many complaints by workers about the unsuitability of their offices for performing VDT-based work. Noise and glare produced by inappropriate lighting systems lead the list of expressed concerns. Workstation 
Table 1. Major concerns of building users.

Issue

Bottom Line Measures

$\begin{array}{lccc}\text { FURNITURE } & \begin{array}{c}\text { Environment } \\ \text { Satisfaction }\end{array} & \begin{array}{c}\text { Job } \\ \text { Performance } \\ \text { (Self) } \\ \text { Layout }\end{array} \\ \begin{array}{l}\text { Comfort } \\ \text { Quality }\end{array} & \mathrm{X} & \mathrm{J} & \\ & \mathrm{X} & \mathrm{x} & \mathrm{x}\end{array}$

AMBIENT CONDITIONS

Temperature/

Air Quality

Lighting

Noise

$\mathrm{X}$

$\mathrm{X}$

PRIVACY

Control Over Access $X$

Intrusions/Distractions $x$

Speech Privacy

Visual Access

$x$

$\mathrm{X}$

COMMUNICATIONS

Ease

Support

FLEXIBILITY $x$

$\mathrm{X} \quad \mathrm{X}$ $\mathrm{x}$

$\mathrm{X}$

The increased popularity of open-office designs has contributed to the problems of office automation because devices such as printers produce considerable noise, thereby disturbing many people near them. The fact that automation typically occurs incrementally in small steps, contributes to the design challenge. Changes are so gradual that there is little anticipation of the final result, and its environmental consequences. For example, adding electronic devices increases a building's heat load, but existing HVAC systems can usually cope with only minor increases in heat. However, the presence of many such systems seriously degrades the quality of the thermal environment, and may require a major HVAC retrofit for an acceptable work setting.

The first automated systems introduced into offices were word processors, designed to increase the efficiency of typing by converting information into an electronically accessible form; establishing the basis for all other systems. The results from the word processing experience pointed to advantages and disadvantages of this approach. While there was some evidence of increased typing productivity, this was accompanied by a variety of complaints, which portended many of those later expressed about the automated office.(5) 
Word processing activities were organized into a "pool" of workers performing a centralized function. A typist had little or no contact with the author of copy, and limited opportunity to carry through many jobs from beginning to end. This process, derived from the factory assembly-line system where work was analyzed, standardized, and simplified to reduce the unit cost of products, resulted in complaints which echoed those of the production-line worker. Workers felt they were subordinated to machines, had limited control over their jobs and little opportunity to interact cooperatively with colleagues. Finally, working eight hours a day at a VDT was a source of concern because of the need for concentration associated with work at terminals.(6)

If the assembly-line system for information processing is inappropriate for performing clerical functions such as word processing, it is even less applicable for automating the activities of knowledge workers. Their tasks are not readily amenable to analytic and standardized treatment, but are characterized by dealing with problems of ever-increasing complexity and a proliferation of information which must be assimilated and acted upon. Their work depends on using highly individualized knowledge and experience, limited and uncertain data in a rather unstructured informational environment. A manager, for example, is given a set of broad responsibilities and considerable freedom to formulate ways of carrying them out. The degree of autonomy associated with this work is one of its principal attractions, and plays a major role in the satisfaction derived in pursuing these careers. To the extent that managers are required to adapt to work methods, technologies and environmental conditions selected by others, they are likely to be less productive, and less satisfied with the quality of their working life (7).

\subsubsection{Technology and Design}

Developments in telecommunications, computers, and office automation systems are changing the office, and the manner in which functions are performed. Information, traditionally developed, processed and stored as paper form, now is processed electronically, while microfiche, magnetic and paper-based systems are used as storage media. New systems are available to conduct activities not possible before. For example, video conferencjng has provided an alternative to face-to-face meetings among geographically dispersed people in the same organization, or among those in different ones. The informational resources available to the individual at a workstation are equivalent to those possessed only by large organizations not many years ago. This has made the workstation the focal point of the office. It serves as the modular design unit, requiring considerable attention by designers.

The linking of individual workstations into networks is of special importance today. It enables individuals to share information and major hardware systems, such as high quality printers. Since office and communications technologies are changing so rapidly, it is virtually impossible to predict how they will evolve in the future. As a result, a major problem for designers is to provide sufficient flexibility for change, at a minimum cost and limited disruption of 
ongoing activities. Among the systems and design strategies meriting attention are:

* Multi-media presentations

* Network connections to all required systems and services.

* Clean vs. utility power.

* Power and localized cooling to accommodate heat loads from electrical equipment and backup such as localized exhaust for heat, heat pump systems to use waste heat, and air-to-air exchange for improved air quality.

* Mechanical, electrical distribution, lighting, and communications systems.

* Workstation design - to accommodate a range of tasks, and individuals.

* Standardization of components for workstations - work surfaces, task lighting, panels, furniture.

* Changes in space use and size.

* Air supply and return, localized and variable in quantity..

* Floor plenum system for air and electrical distribution

* Storage of informational media -reduced redundancy for paper, disks.

* Convenience copiers and printers.

* Fire suppression and safety systems.

* Ratio of devices to people.

At the macro level of design, available systems can be automated and pre-wired into new buildings to accommodate operational, service, energy conservation, fire safety, communication, and other systems. However, just because technology exists to automate functions doesn't mean that it is the most effective approach for the organization or the individual worker.

Automation is typically based upon uniform requirements, while individuals and activities often differ from one another in important ways, and have distinctive needs. The design approach to the automated office today is directed toward meeting the needs of the average office worker performing typical tasks in a characteristic organization. This depiction of the automated office is both widespread and meaningless. There is no such thing as a typical:

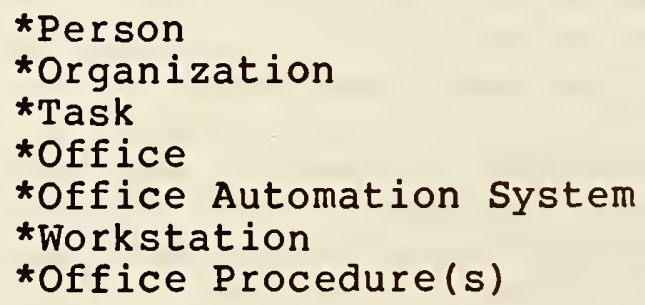

Organizations and individuals have unique characteristics which merit design attention, and it is the function of the design process to develop the information needed to respond to the particular of requirements of both of them. 
Architects have an important contribution to make in furthering organizational and group cohesiveness.

Microcomputer-based workstations have enabled many jobs to be performed autonomously, isolating the individual from a working group and from the organization as a whole. Design elements such as furnishings and panels can serve to distinguish one group from another one, and the layout of workstation groupings can be integrated to further organizational functions. Common spaces can provide the opportunity for members of diverse groups to exchange ideas, thereby furthering the sense of organizational identity. Above all, there is a need to provide a high quality environment for the staff. Table 2 suggests several features which might contribute to "high quality" design.

Table 2. Checklist - Design Considerations for 'High Quality' Offices (Criteria)

* Humane and pleasant setting to offset technological emphasis.

* Places for visual relief, inside and outside.

* Spaces arranged to make locations and circulation patterns comprehensible.

* Places for casual interchange of ideas and unplanned social contact.

* Centers of interest to serve as guideposts and informal meeting places, e.g. works of art, planters.

* Quiet, restful areas, with expanded recreational and physical exercise spaces.

* Workstations grouped in functional clusters.

* Personalization and individual control of workspace furnishings and environmental systems.

* Attractively designed public areas.

* Privacy - visual and auditory.

* Secure spaces.

* Individual choice of workspace features; lamps, furnishings

Organizational issues are closely linked to technological, ergonomic, and design factors. Technological changes in office systems have been too rapid for them to be properly assimilated into most organizations. This has resulted in a gap between what is technically feasible, and what is needed to further organizational goals. 
Automated technology is neutral with respect to its influences on the quality of the workplace. It can be used as a means of standardizing and controlling job characteristics and environmental features, thereby minimizing the autonomy of the individual worker and the freedom to make on-the-job decisions. Many of the problems with the early automated word processing have been attributed to these factors. On the other hand, automation offers the potential for the individual worker to exercise considerable control over job functions and environmental systems, thereby enhancing the quality and quantity of work performed.

Individual control can take many forms:

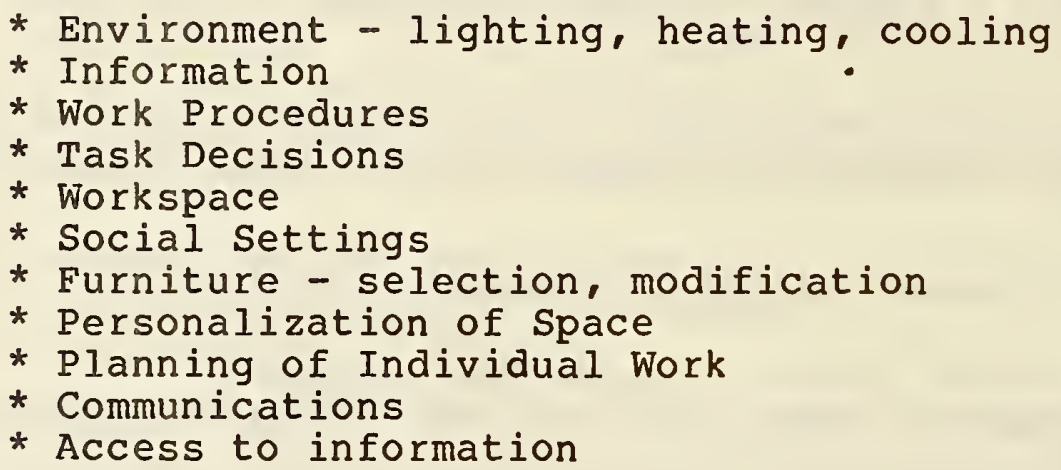

\subsubsection{Security and Design}

As communication, information and building technologies become more dependent on computerized systems, a greater likelihood exists that when there is a problem, it will be a major one. That is, since many systems are interdependent, a breakdown in one system often has widespread and costly effects. This situation makes highly automated buildings and the activities they house, vulnerable to people who want to undermine, disrupt and destroy operational information. Building security is therefore an important design consideration.

The greatest need for security in office-based operations is that required in a data center, where informational resources are highly centralized. In chapter 15 of this report, security in data centers is treated in some detail. The requirements in general offices are likely to be less stringent and therefore the design requirements are are not as comprehensive as those in data centers. However, the methods employed are not apt to be qualitatively different in the two spaces. Rather, they would differ only in degree.

\subsubsection{Planning}

Productivity in offices results from the appropriate integration of people, tools, and places. This integration should be accomplished by proper planning, including organizational representatives from human resources, technology, information processing, and design who often find themselves in competition with one another in productivity improvement programs. The planning should be under 
the direction of top management and include extensive involvement by end-users at all stages of the process (8).

The automation of office functions is a continuous process, not a discrete event, and must be directed to organizational effectiveness and not the introduction of new technologies because of their advertised effectiveness. An essential element in such planning is the preparation and training of the staff to ensure that automation is viewed as a tool to assist work activities, not a threat to jobs, or a means to subjugate people to technology. It is self-evident that the greatest single determinant of office productivity is the performance of the workforce. Technology as well as design should contribute positively to the office work setting (9). 


\section{PLANNING AND PROGRAMMING THE AUTOMATED OFFICE}

\subsection{FUNCTION}

Planning is used to promote the orderly transition of office work from primarily paper-based activities to electronic systems while maintaining (or improving) the overall quality of the workplace. This goal can be accomplished by acquiring detailed information concerning organizational, individual and technological

requirements and then devising appropriate design solutions. The planning should be accomplished by the close cooperation of a multi-disciplinary design team with the client (10).

Designers and office automation experts are in general agreement that detailed planning is an essential prerequisite for the automation of office activities. The piecemeal acquisition equipment has resulted in many complaints by office workers in the past, because of such factors as the inappropriateness of furniture and environmental systems (e.g. lighting) for performing VDT-based activities (11). A key goal of planning is to ensure the flexibility required to accommodate future changes.

\subsection{CRITERIA}

* Planning should be under the auspices and commitment of top management.

* End-users should be an important source of information. * Flexibility, and its costs, should be major considerations.

* The integration of information and communication systems is important.

* The analysis of present and proposed office activities as they meet organizational objectives should precede any decision about automated equipment and systems. * Long-term costs should be considered - especially those associated with the salaries of workers.

* Degree of change forecast.

\subsection{INFORMATION DEVELOPMENT}

A variety of approaches have been used as planning aids. Some of these are illustrated in tables 3,4 , and 5 . 
Following (Tables 3 and 4) are two methods which can be used to develop planning information:

\section{Table 3.a. Analytic Approach for Relating Technology to Design (12)}

PRESENT OFFICE

INFORMATION/MANAGEMENT SYSTEMS

Record Storage and

Management

Internal Ma11 Generation and Distribution

Document Distribution

Document Preparation

Organization of Activities

Expansion of Capabilities

Planning Activities

AUTOMATED OFFICE

POSSIBLE DESIGN IMPACT

Limited paper

Electronic files

Paper

Typed copy

Separated functlons; Manual typing, artwork graphics

Speclalized Decentralized

Space set aside

Minimal necessary
Centralized functions Electronically based systems

Workstations - clerical professional, managerial

More power, wiring, ducting, to accomodate future needs
- Less space for file cabinets

- Changed layout

- Added electronics

- Added cooling load

- Smaller mall room

- More electronics

- Need for physical separation of noisy machines \& activities

- Special acoustic treatment

- Larger workstation for document preparation

- Less space for visual arts

- Change in adjacencles

- More electronics

- Greater access to data bases

- Added capacity for electronics, power, plan for local cooling
Detalled planning essential; - Need for detalled architecManagement, bullding, Information, communication, etc tural program, CAD system to keep track of changes, etc. 


\section{Table 3.b. Analytic Approach for Relating Technology to Design (Cont'd) (12)}

\section{POWER SYSTEMS}

Emergency Power

Wire Distribution

OFFICE TECHNOLOGY

BASED ACTIVITIES

Typing

Communication

Filing

Facsimile

Production

Read1ng
None

\section{Conduits}

Under floor systems

In wall outlets

Electronic typewriter

Telephone, ma1l, memos

Paper files; individual

Hands-on duplicating

Paper copy
Batteries, Generator

Support for electrical

equipment during outage

Ralsed floors

Cellular decks

Flat conductor cable
- Added space, special venting systems, fuel storage, special flooring for ac1d, fuel runof

- Fire protection, security

- Clean power

- New floor systems; carpets

- More space for floor/celling systems

- Changed power loading factorsless load per local circult, more cable runs

- More room for wire closets, risers

- Special protection for cables

Word processing systems

- Glare free lighting

- Additional workspace for equipment

Electron1c ma1l

- VDT, wiring,

Electronic data bases Centrallzed computer

- Spec1alized fac1l1tyralsed floor, clean, emergency power, less paper storage space

Duplication at a distance, FAX

- Specialized facility for devices; acoust1c treated, separate air conditioning

- Special light1ng, more desktop room for equipment 
Not applicable

Not Aware
No

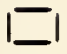
professional or supervisory counceling or confidential discussions on a regular bas18?

2. Have conference spaces been provided for those t1mes when employees need to work together?

3. In circumstances where ceveral employees share equipment, are those employees co-located?

4. Are the employees separated from nolsy equipment such as CPUs, coplers, etc?

5. Have office locations been chosen with regard to expected 1riteract 1ons?

6. Are partitions used to screen traffic?

7. Are all comonly used facillties centrally located?

8. Are frequently interacting functions located on the cane floor?

9. Are the pathways to and from off 1 ces stralght? (That 18, are confusing spirals, curves, or dead-ends avolded)?

10. Where an open office concept has been used, is some amount of pathway continuity present?

11. Are the dimensions of all private offices at least $7.5 \times$ $7.5 \mathrm{ft}(2.3 \mathrm{~m} \times 2.3 \mathrm{~m}) ?$

12. Are the private off1ces designated so no one faces a window while working?

13. Does each private office have a good source of 1llunination?

14. Does each office have independent control of Its vent1lating system?

15. Is there adequate opace at the vorkstation for the equipent and data (printouts, acrofiche, anuals, etc.) that the user needs?

16. Is the work area adjustable so that 1t can accomodate comfortably the 5th to the 95th percentile of the adult population?

17. If the CRTs, the microf1che readera, etc., are not at the Imedlate workstation, are they as cloae as poselble to that station?

18. Is the workstation adjustable so that the hove typing keys are 2 Inches $(55 \mathrm{~mm}$ ) below the elbow helght?

19. Are all employees trained to adjust their furniture, or 18 adjustment provided as requested?

20. Are office no1se levels below $45 \mathrm{~dB}(\mathrm{~A}), 37 \mathrm{~dB}$ PSIL-4, or 38 dB PSIL? 


\subsection{PLANNING APPROACHES-PROGRAMMING}

The information developed during the planning phase will serve as the basis of the architectural program.

Objectives and Philosophy. A clear statement of goals is the first step. These are based on the history and background of the organization and its attitudes and expectations towards its client and staff.

Functional Relationships. An analysis of the interactions and transactions of individuals and organizational units is needed to understand traffic flow, communication, information flow and access. It is also necessary to determine the appropriate placement of support services such as word processing, mail reproduction, data storage and communication, etc., with relation to the location of administrative and organizational units. The proper location of conference and meeting rooms, libraries, internal waiting areas, lunchrooms, lounges, all merit particular attention.

Facility Spatial Requirements. Spatial needs for individual and organizational units are based upon a complex of interfacing factors such as ergonomics, communication requirements, status, and needs for privacy.

Development of the Program and Program Criteria. The program should be written in performance language, describing the functional requirements of a system for example, not the hardware specifications.

Post Occupancy Evaluation (POE). Establish at the design stage, individuals responsible for POE maintenance; as-built drawings review, training, etc. To prepare for future evaluations, a log of projects should be kept and preserved, including all plans, the program, decisions and changes made. Evaluation should be made on the basis of general staff satisfaction, staff use of space and facilities, environmental and communications systems, task performance, and the value and use of staff amenities.

Following (table 5 and figures 2, 3 and 4 ) are approaches that have been used to develop and organize programming activities, including post occupancy evaluation (POE). 
Table 5. Introduction of Automation into Offices-Critical Factors

1. Collect facts, to document automation approach.

2. Start early to announce and explain system, answer questions, share plans, generate enthusiasm and gain long term commitment.

3. Share information with management, support staff and end users.

4. Implement systems gradually; pilot test systems, including factory tests, mockups; communicate results to staff.

5. Be aware of "grapevine" information; make informal network work for you.

6. Collect information during entire process; share results.

7. Identify and resolve problems quickly; use experts if needed.

8. Use informal meetings to discuss ongoing developments; maintain staff enthusiasm.

9. Know when to turn operation over to user group; with documented procedures, and information about available assistance.

10. Involve management and end-users in process.

11. There should be an information center for on-going training and help.

12. There should be a 'help' desk to resolve problems.

13. A systematic change control system should be instituted.

14. Information should be solicited and shared with individuals, not just formal organizational groups. 

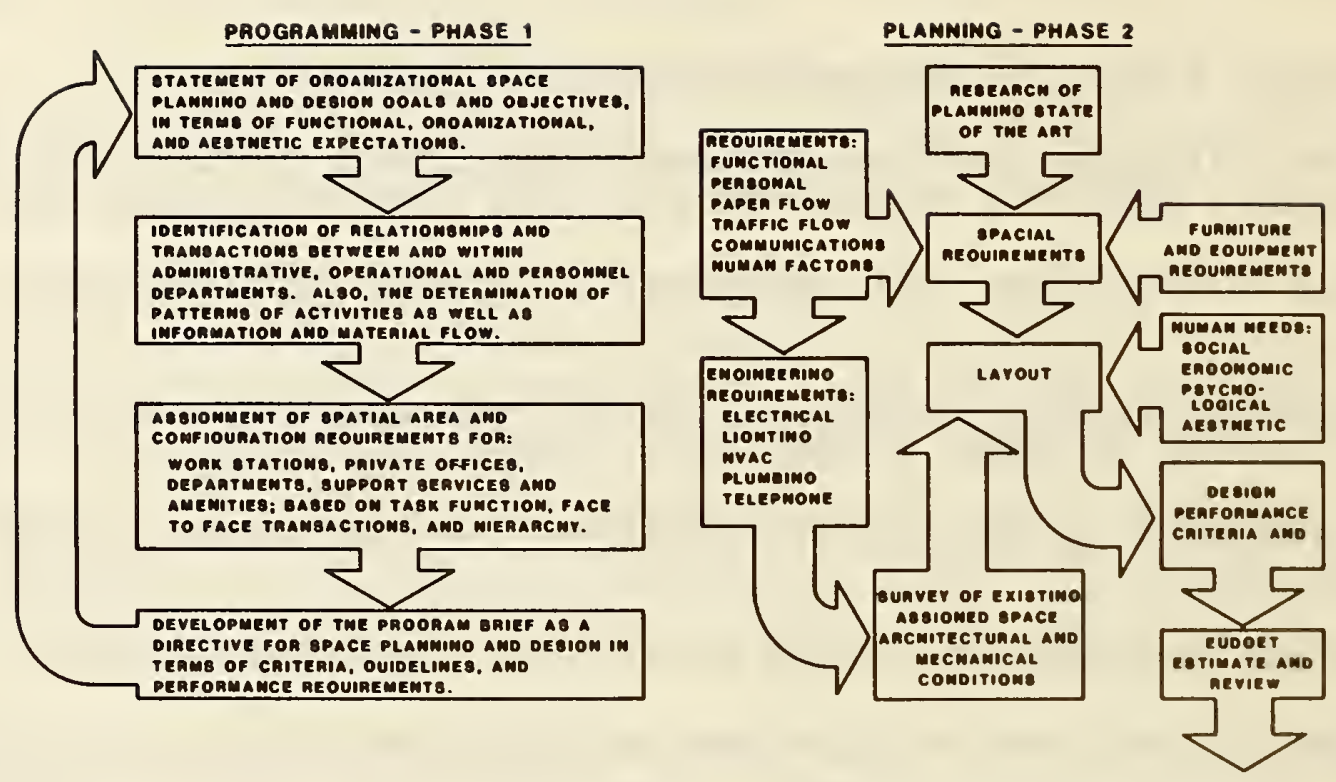

TECHMICAL - PHASE 3

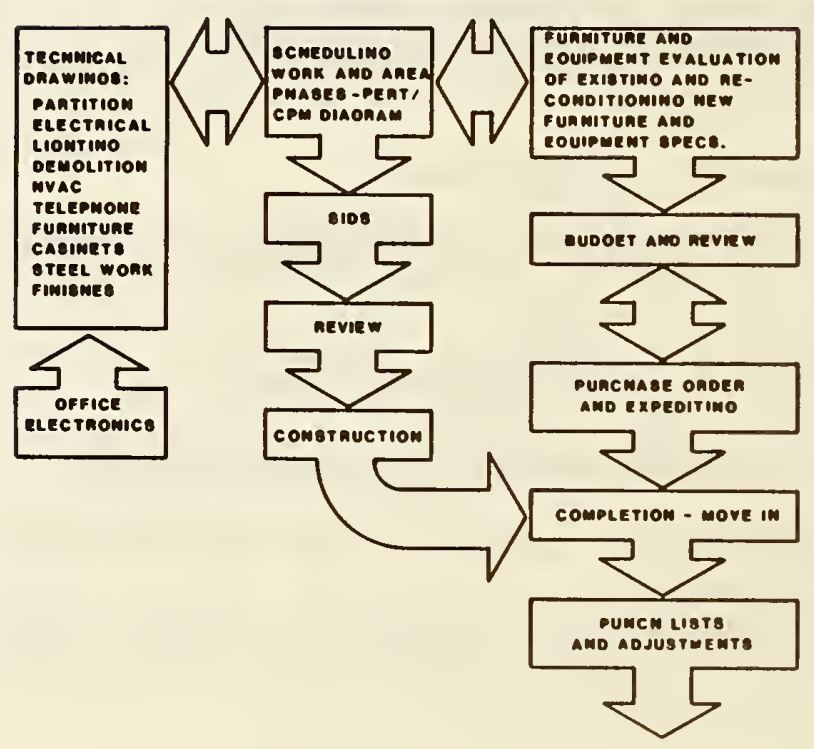

EVALUATION - PHASE 4

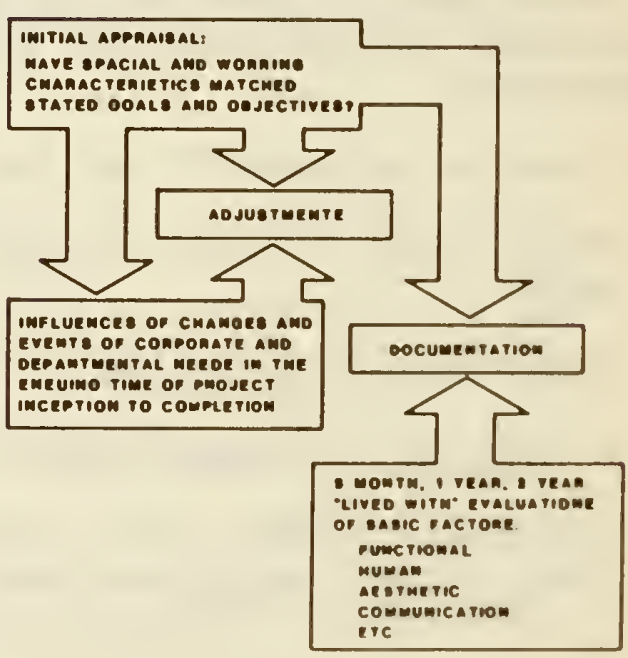

Figure 2. Office Design Process (14) 


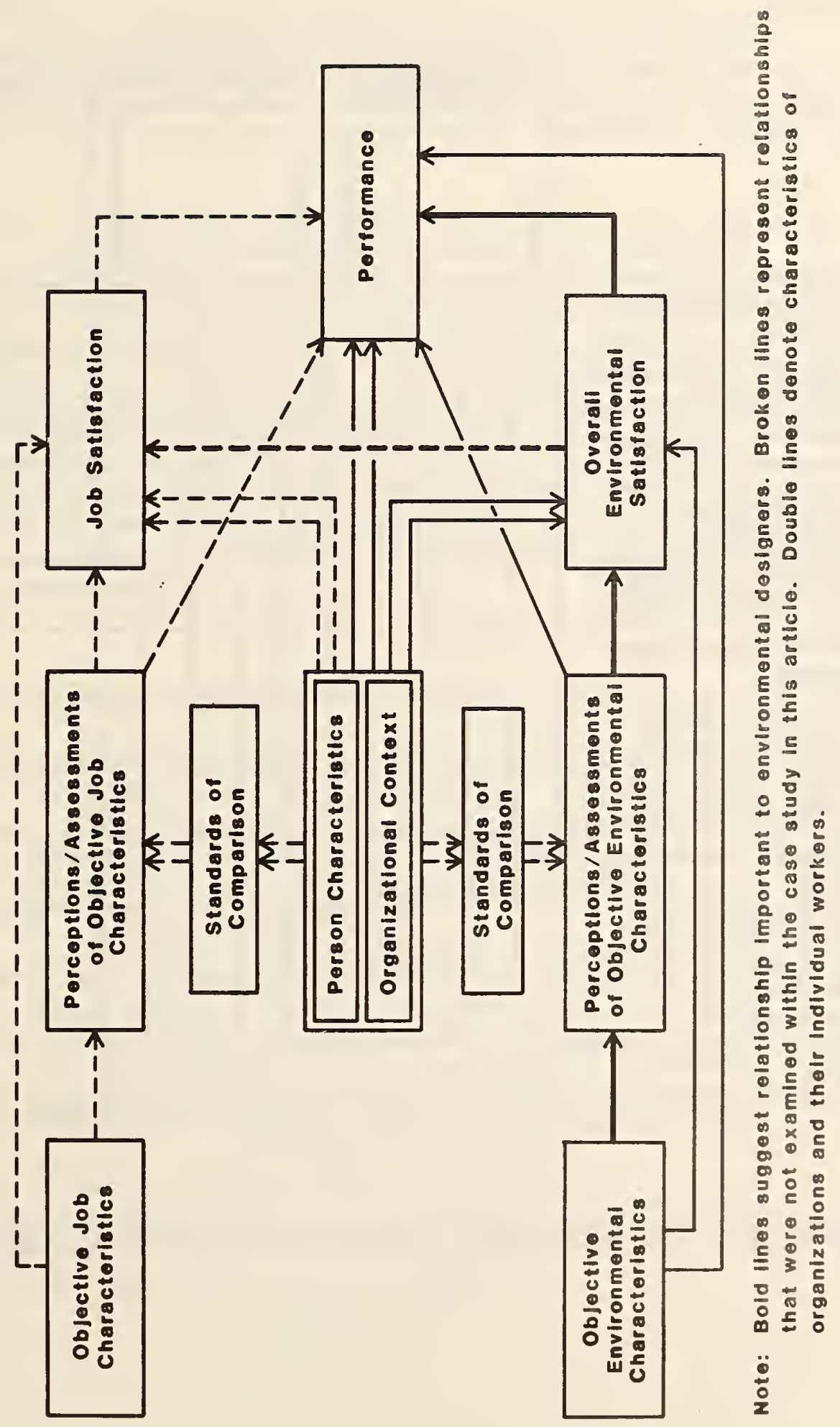

Figure 3. Conceptual Model for Evaluating Work Environments (15) 


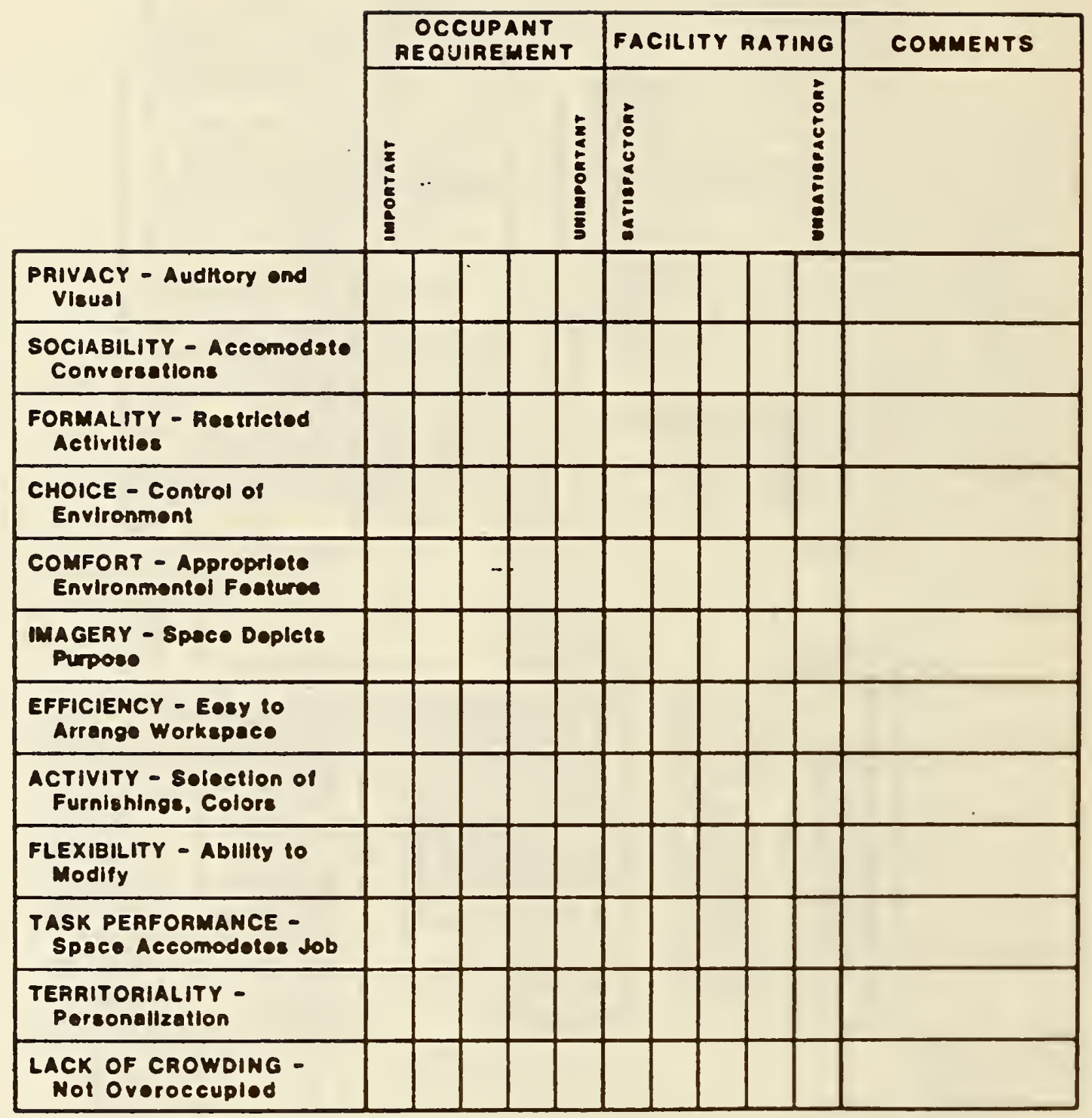

Figure 4. Checklist for Facility Evaluation (16) 


\subsection{FUNCTION}

The workstation is the basic operating unit of the automated office. It is here that information and communication technologies are merged into a powerful system managed by the worker. Initially confined to secretarial and data processing tasks electronic devices are increasingly used for managerial work.

Organizational, design and personal needs are integrated at the workstation. It must be designed to meet a variety of requirements differing from person to person- physical, sensory, and cognitive. Flexibility is essential for workstation furnishings and equipment. For example, selecting and placing objects serving the worker requires considerations of physical comfort, body movement, and individual preferences and needs. Employees at workstations must have ready access to the tools and materials needed to do their jobs. Size of components, clearances, allowances for free body movement, are integral to the proper fit of person to workstation. Figures 5 and 6 , and table 6 are examples of recommended designs and dimensions of workstation components.

\subsection{CRITERIA}

The workstation should have:

* Flexibility to accommodate the range of tasks performed and the individuals performing them

* Vertical and horizontal surfaces for materials use and storage; place for continuous paper supply and output * Power and communications capabilities consistent with performing required activities

* Ready access to work materials

* Workstation layout to facilitate performing activities

* Individual controls for equipment and selected environmental features, e.g. task lighting

* Capability for personalizing workspace

* Easily reached controls; easily read displays

* Sufficient space to facilitate comfortable movement

* Capability for rapid changes and moves

* Appropriate wire management and media for tasks

* Job related space for visitors, paraprofessional; e.g. meeting areas

* Antistatic materials on work surfaces 
Table 6. Recommended Workstation Dimensions for VDT's (74) STD $1472 \mathrm{C}$

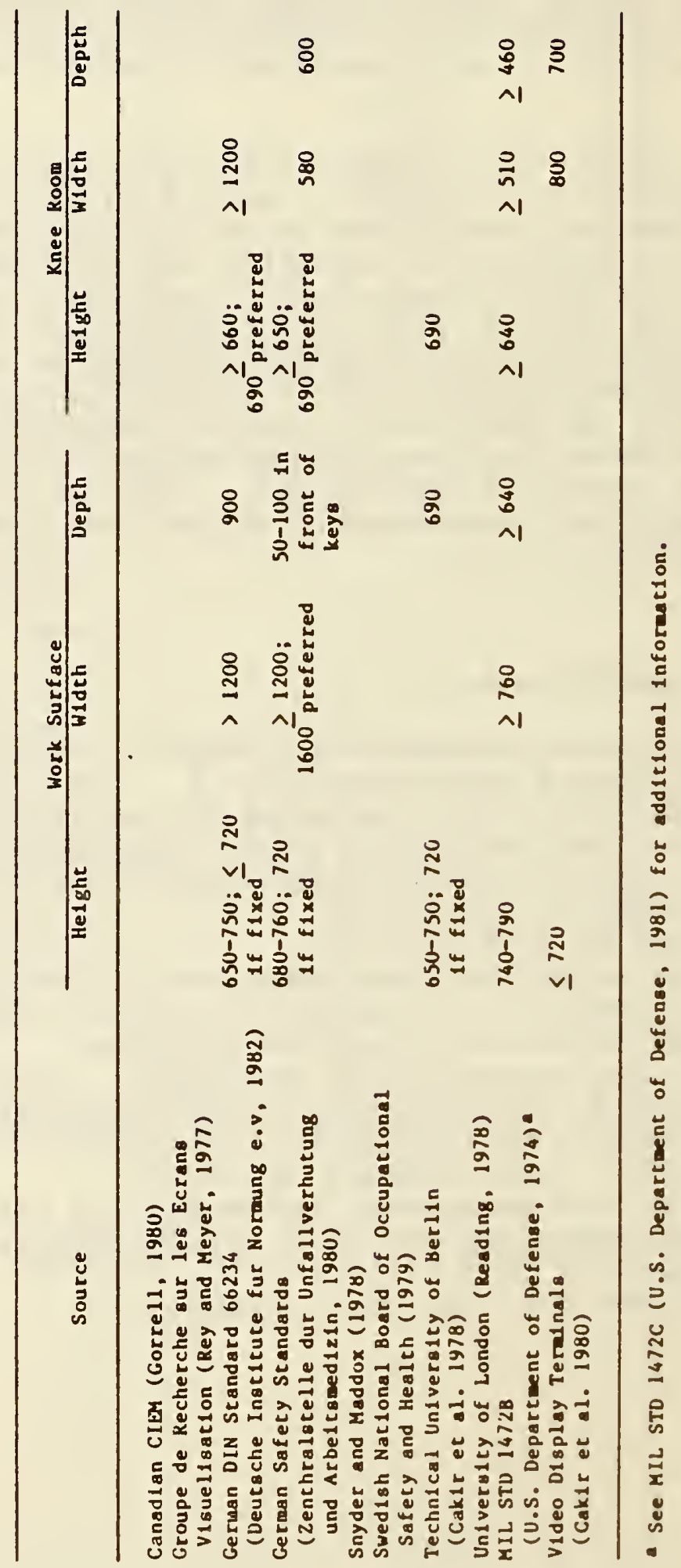




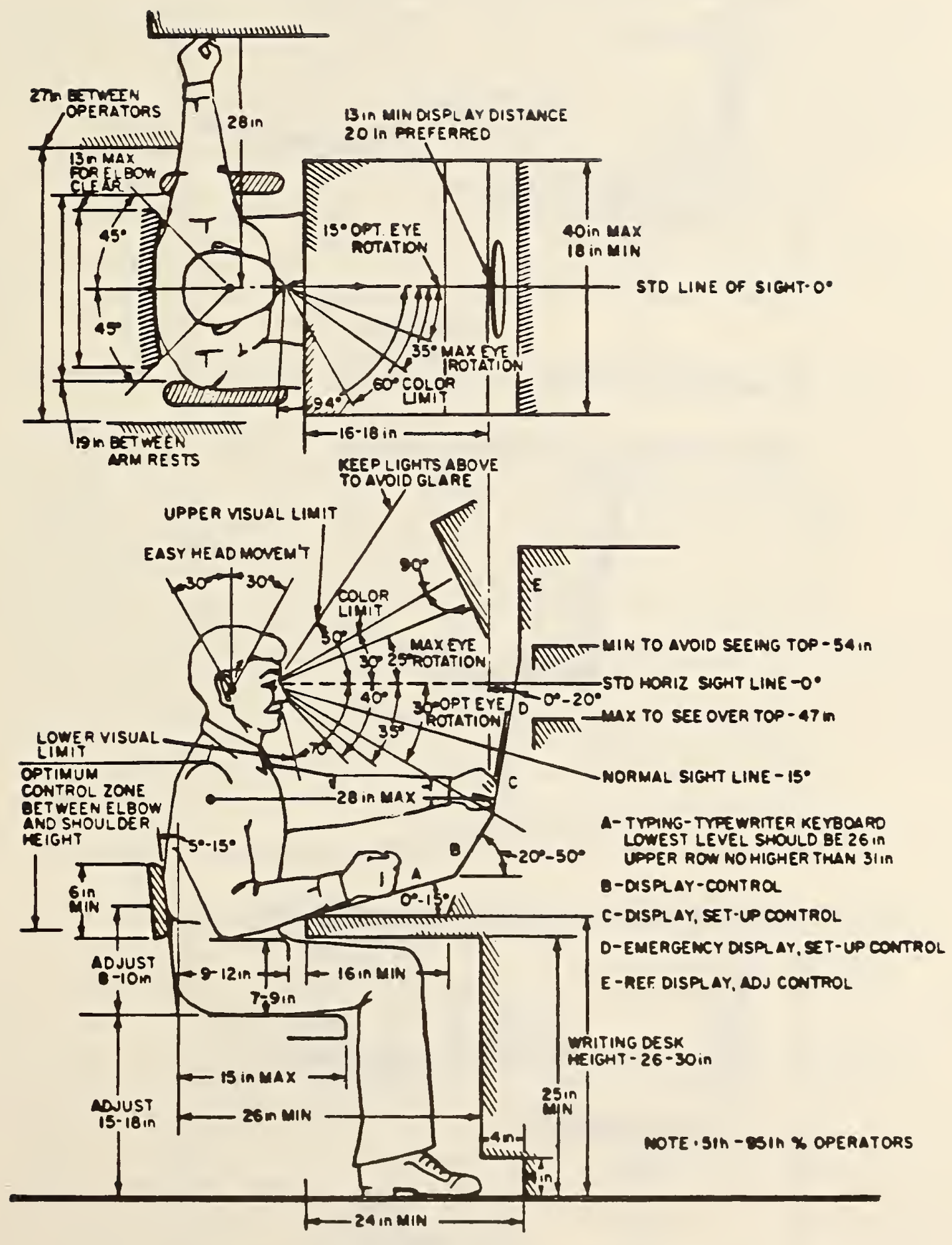

Figure 5. Ergonomic Criteria for Workspaces (17) 
Aocommended Approximate Otmendens (in centinoters)

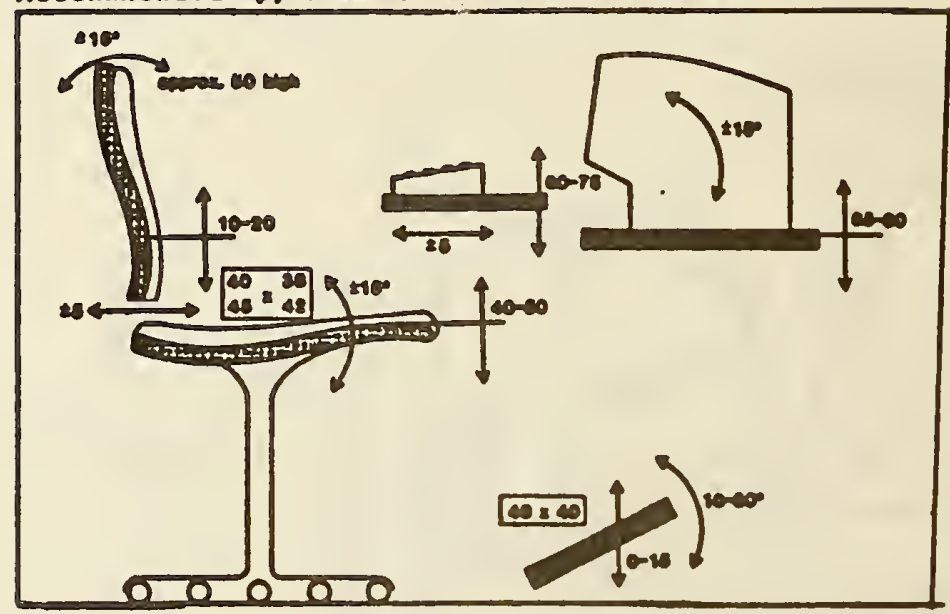

Adjustmont Fodtures of en Ergonomic Wort soot

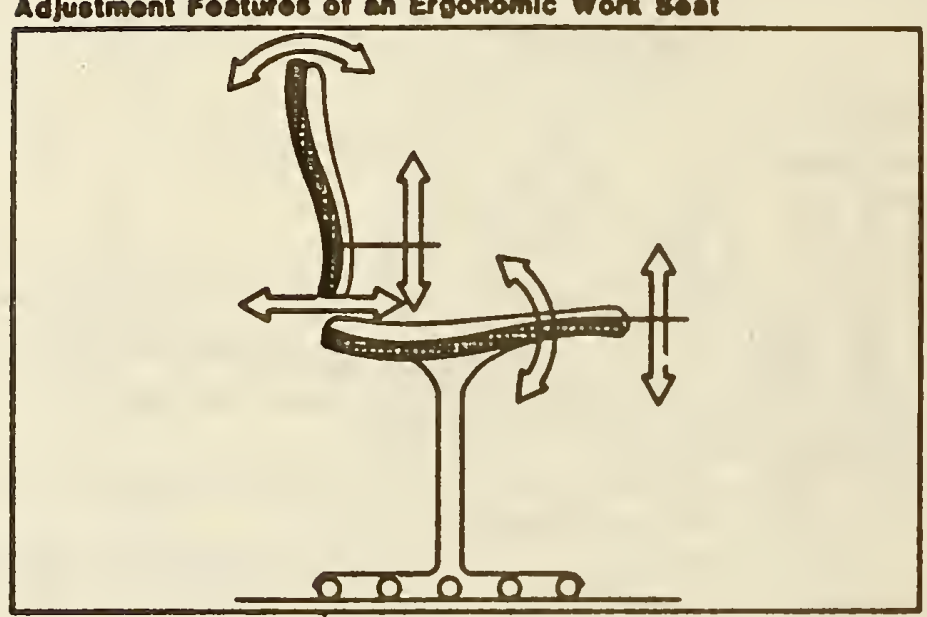

Adpotment Features of en Erconomic VoU Work Station

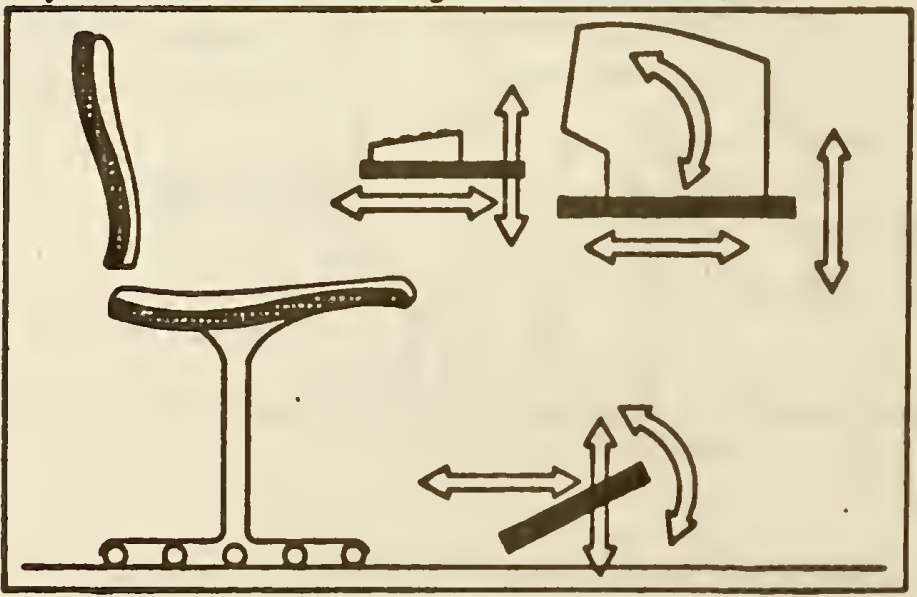

Figure 6. Workstation and Seat Design 


\subsection{FURNISHINGS-FLEXIBILITY REQUIREMENTS}

The concept of the 'average person' has been discarded as a guide for effective design. Individuals have a wide variety of sizes and shapes, and have different preferences for equipment and furnishings. Just as the design of the office must be flexible, furniture must be adjustable. Seat, keyboard, display, and foot support are interacting system components in workstation design. Chairs should be supported on four legs (or a pedestal with a five caster base), have adjustable seat pans and backrest heights. The angles of seat pans and backrests should be adjustable relative to each another. Backrests should also be modifiable front and back, with respect to the seat pan. Footrests, adjustable in height, angulation, and front and back distance are also desirable (18).

\subsubsection{Checklist -- Requirements for Workstation Flexibility}

* Capable of change with minimum disruption to staff or activities

* Adjustments should not require special skills or tools.

* Systems should be simple and made of standardized components and cost effective.

* Dimensions and heights of work surfaces should be adjustable and accommodate a range of activity and individual requirements.

* New component configurations should accommodate changing requirements.

* Furnishings should be responsive to individual

differences, e.g., left and right handed people.

* Adjustable seating with firm back and neck support

* Components and design should be cost effective

\subsection{ENVIRONMENTAL CONTEXT}

3.4.1 Checklist for Workstations Environment/Design Components

* Are components adjustable; fit differences in height, size, and work habits? Can products be used in a right and left handed configuration?

* Is the hardware adaptable? Can the system be shifted, moved on a carpeted floor? Is it readily adjustable with minimum expertise and simple tools?

* Can the equipment be used in angular configurations?

* Does the system optimize the use of vertical space?

* Do acoustical panels work properly?

* Does the product control, modify, and conceal electrical and communications systems?

* Are visual privacy needs satisfied?

* Can the worker control the mini-environment?

* Is the product durable, safe, and easy to maintain?

* Is the product standardized; available if additional components are needed? 
* Do components relate visually to one another and the other system elements?

* Is a "family" of products available, e.g., different but compatible colors?

3.4.2 Checklist - Requirements for Workstation Environment/Design

* Acoustic privacy.

* Freedom from auditory and visual distractions.

* Glare-free lighting.

* Physical separation of unrelated activities.

* Ease of power and communication changes and additions.

* Nondisruptive traffic patterns.

* Clearly defined personal boundaries.

3.4.3. System Panels

System panels in open office settings perform the functions traditionally served by walls in private offices.

\subsubsection{Function}

Systems panels are important in open-office design. They are used for acoustical and visual privacy, articulating office layout and facilitating wire system distribution. They help to implement changes in the office environment. Panel heights vary from 30 inches to 80 inches (where acoustical and visual privacy requirements predominate, such as in executive workstations). Lower partition heights produce improved communication and air circulation at the expense of privacy. Higher partitions aid acoustical performance, but create difficulties for HVAC and lighting systems.

An analysis of requirements should precede the selection of panel systems. For example:

* Will open planning be used for all staff members? What are the priorities?

* How important is the ability to add units, wire management?

* Will sophisticated computer equipment have to be accommodated?

* Does company policy dictate standards for management levels? 
Structurally, panels can be placed in one of five categories:

1. Free standing; serves as a space divider.

2. Panel System with End Support; stabilizes work surfaces.

3. Cantilevered; structural element that can support

furniture. Work surfaces and overhead storage cabinets, which can be raised and lowered are hung from the back panel, usually on slotted tracks.

4. Post and Panel; supports overhead cabinets and work surfaces. The posts enable panels to be set in any configuration.

5. Full Wall Height; for privacy. They come with upholstered, wood or glass panels, and full height doors.

Panels are constructed with metal or wood frames, with or without exposed or integrated supporting posts. Connector systems vary widely. Panel material composition is fairly standardized. Nonacoustical panels are often made of hardboard or honeycomb cores sandwiched between layers of laminate, wood veneer or metal. Acoustical panels may have a hardboard core with layers of fiberglass or other sound absorbent material on both sized, covered by a layer of acoustically transparent fabric.

\subsubsection{Panel System Criteria}

The following features of panel systems are desirable:

* Acoustical performance consistent with activities

* Wiring distribution - current and future media

* Capable of being readily disassembled and reconfigured with unskilled labor

* Sufficiently durable for repeated reuse

* Standardized components to ensure availability of replacement and expansion

* Appropriate range of sizes, colors, etc to meet organizational needs and plans

* Adequate stability and impact resistance 
3.4.3.3 Checklist for Panel Components

* Are components adjustable to fit individual differences in height, size, and work habits

* Can the product be used in right and left handed configurations?

* Is the hardware adaptable?

* Can components be moved, shifted on a carpeted floor?

* Can adjustments be made with simple tools and by those with minimum expertise?

* Are angular configurations possible?

* Is there optimum use of vertical space?

* Do acoustical panels perform as desired?

* Can electrical and communication systems be controlled, modified and concealed?

* Do they adequately serve visual privacy needs?

* What control does the worker have over the minienvironment; e.g. lighting, cooling ?

* Are the products durable, safe, and easy to maintain?

* Are the products standardized; available if added components are needed?

* Is there a family of products; e.g. compatible colors, unit sizes?

* Are there sound or light leaks between connected panels?

* Are there slotted rails along the face edged to hang storage and workspace components?

* Is there provision for a coat rack?

* See Table 7 
The following table describes minimum federal performance requirements for panels: Table 7

\begin{tabular}{lll}
\hline ATTRIBUTE & TESTS & $\begin{array}{l}\text { MINIMAL FEDERAL } \\
\text { PERFORMANCE REQUIREMENTS }\end{array}$ \\
\hline FLAMMABILITY & & \\
\hline FLAME SPREAD & ASTM E-84 & $\begin{array}{l}\text { FLAME SPREAD }<25 \text { UNSPRINKLERED } \\
\text { FLAME SPREAD } 200 \text { SPRINKLERED }\end{array}$ \\
SMOKE & NBS 708 & SPECIFIC OPTICAL DENSITY<150 \\
POTENTIAL HEAT & $\begin{array}{l}\text { BASED ON ASTM } \\
\text { PROCEEDINGS VOL, 61 } \\
\text { 1961, PP 1337-47 }\end{array}$ & $<40,000$ BTU \\
& &
\end{tabular}

ACOUSTICS

AIRBORNE SOUND

PBS-C1

(SUBJECTIVE)

SPP $<60$ IN OPEN PLAN AREAS

SPP $<70$ IN ROOMS WITHOUT DOORS

PBS-C2

(OBJECTIVE TEST)

NIC $+(N C<40)<60$ IN OPEN PLAN

IMPACT SOUND

FOOTFALL TEST

1B1-1 1-1965

MASK VERTICAL FOOTFALL SOUNDS $\mathrm{W} / \mathrm{NC}<40$

STABILITY

RESIST HORIZONTAL ASTM 72-74

RESIST LOAD OF 50 LB

LOAD

SUPPORT 40 LB PER LINEAR FT OF SHELVING

RESIST TIPPING

RESIST IMPACT

ASTM $72-74$

RESIST 30-LB IMPACT HORIZONTAL FORCE OF 100 LB APPLIED 5" ABOVE FLOOR

\section{DURABILITY \\ ABRASION}

COLORFASTNESS

CHEMICAL RESISTANCE
ASTM $72-74$

FADEOMETER

FTM 141 A METHOD 6081
RESIST ABRASION

NO COLOR CHANGE AFTER 50 HOURS AT $150^{\circ} \mathrm{F}$

RESIST STAINING AND DAMAGE

SOURCE: Specifications for Commercial Interiors, by S.C. Reznikoff, New York, WHITNEY LIBRARY OF DESIGN, 1979. 
3.4.3.4. Interactions with other systems

Wiring

The system panel plays an important role in electrical distribution systems in open offices. Three basic approaches are used in wire distribution.

* Panel with a raceway that accommodates electrical wires and communication cables of all kinds, including coaxial, mixed media.

* Panel which can be equipped with an optional manufacturersupplied power pack, panel-to-panel connectors, and hooks up to the building"s electrical grid.

* The prewired panel, already assembled and plugged into the electrical grid. (The most expensive option.)

\section{Simple Raceway}

This system is assembled before the panels and adds a few inches to the total system height. Among the specific issues to be considered are:

* The accessibility of wires and cables

* The durability of base plates and covers concealing the wires

* Are separate channels available for communications and electrical wire?

* Availability and accessibility of power feeds from the floor and ceiling

* Are there optional convenience outlet strips?

* Difficulty in managing wire; replacement, making changes.

Power Pack

Power availability is a major concern for all wiring systems. Among the factors to be considered are:

* Wiring system retrofitted to non-electrified system panels

* Integrated convenience outlets at work surface height

* With systems having integrated ambient lighting, can the lighting circuit be turned off while task and convenient lighting are active?

* Pass-through panels for areas not requiring electrified outlets

* Number of outlets permitted in each circuit

\section{$3.5 \mathrm{VDT} / K E Y B O A R D$ SYSTEMS}

The primary working tools at the electronic workstation are the VDT and associated keyboard. The system should be easy to use and adaptable to individual requirements, e.g., its ergonomic features. 
3.5.1 Checklist-VDT/Keyboard Requirements:

* Movable and connected keyboards for individual convenience and/or preference

* Readily adjustable VDT screen- capability to tilt, move vertically, swivel toward or away from the operator

* Keyboard enabling operator's hands and elbows to be in comfortable positions

* Work surfaces at preferred height and distance for individual ease of manipulation, support or forearms, palm and wrist, and convenience for seeing the task.

\subsection{POSSIBLE PROBLEM AREA-VDT SCREEN GLARE}

A major complaint by VDT operators has been screen reading difficulty due to glare. The lighting and design issues related to this problem are covered in Chap. 5). A study by the National Academy of Sciences suggests the use of filters to enhance visual image quality on the VDT screen. It describes four systems available for this purpose.

3.6.1. Potential Solution - Filters over VDT screen.(18)

* Circular polarizer with anti-reflective coating, used to reduce specular and diffuse reflections. The outside surface is coated with several layers of optically transparent materials to form an anti-reflective coating to reduce specular reflections from the filter surface. The remainder of the filter package is a polarizer to eliminate reflections from the underlying VDT screen.

* Neutral density filter is the simplest type, consisting of neutrally tinted plastic allowing a small percentage of light falling on it to be visible. It reduces diffuse light reflections from ambient sources.

* Notch or color filters allow transmission of a high percentage of lights from certain wavelengths (usually green), and absorption at other wavelengths. It is designed to be "tuned" to the color of the VDT screen, permitting the desired light to penetrate, while absorbing the undesirable ambient light.

* Directional filters employ geometrical means to prevent ambient light from reaching the screen, thereby preventing reflections from reaching the user. 
A summary evaluation of ergonomic features of offices revealed the following: (20)

GENERAL FEATURES

RANK

Comfort

Amount of workspace

Ability to adjust furniture oneself

Amount of storage space

Ease of adjustment

Amount of light available

Privacy

Noise control

DESK CHARACTERISTICS

Writing surface height adjustment

Terminal screen height adjustment

Terminal keyboard height adjustment

Separate heights; keyboard and screen

Terminal screen tilt

File drawer storage

Shelves

Terminal keyboard tilt

Terminal viewing distance adjustment

Locking storage area for personal items

CHAIR CHARACTERISTICS

Seat height adjustment

Back rest height adjustment

Ability to swivel while seated

Back tilt adjustment

Arms

Seat tilt adjustment

Ability to lean back

Carpet casters

Footrest 


\subsection{FUNCTION}

To provide an acoustical environment appropriate for the activities performed in automated offices.

The auditory needs of workers depend upon the characteristics of the work performed. For example, many managerial and professional functions have stringent acoustical demands and are performed by people accustomed to a quiet workplace. A manager is often required to conduct private conversations with staff members and visitors. Professionals spend considerable time in planning, creative thinking and report writing. These activities are susceptible to distraction from noise intrusions. On the other hand, many office tasks require office machines and systems inherently noisy, such as copiers and most printers. One design challenge is to physically separate or isolate activities with conflicting acoustical properties.

\subsection{BACKGROUND}

Surveys consistently demonstrate that the acoustical features of office are extremely important to most employees, especially for those working in open-space design offices. Studies also indicate that major sources of complaints are intrusive noises and the lack of acoustical privacy.

With respect to speech privacy, confidential discussions play an important role in office activities. The general public is often served by private and public organizations in open-office environments. The subjects of many discussions are highly personal and confidential, and should not be in danger of compromise.

Another problem is lack of effective noise isolation from noise producing equipment. Unlike the situation in a private office where such noises are largely confined to a specific work area, in the open-office design these noises frequently intrude to many work areas where individuals require a quiet environment to engage in analytical activities. Noise has a detrimental effect on the ability to concentrate on the job at hand, especially for management and professional personnel.

The acoustical properties of spaces are influenced by the thickness and density of enclosing structures, the porosity of wall and floor covers, insulation, drapes and furnishings. Sound is affected by room shape, volume, and the characteristics of the interior surfaces. 


\subsection{CRITERIA}

A proper acoustical environment consists of:

1. Adequate acoustical privacy; consistent with the activity performed.

2. Freedom from intrusive noise sources.

3. Freedom from distracting vibrations.

4. Ability to conduct conversations at normal speech levels.

5. Isolation of major noise sources from general working environment.

6. Special acoustical treatment for sensitive activities/spaces, e.g.conference, audio-visual rooms. 7. Control of telephone rings; ensure that $r$ ing of one phone is identified and not confused with nearby telephones.

\subsection{OVERVIEW OF BASIC ISSUES}

Before discussing design approaches to achieve adequate acoustical performance several basic issues and concepts will be mentioned. Table 8 indicates background sound levels of familiar activities and places.

Table 8. Familiar Background Sound Levels

\begin{tabular}{|c|c|c|}
\hline Apparent Louthes & Examples & Dectibut \\
\hline \multirow[t]{2}{*}{ DEAFENING } & Je aircreft & no \\
\hline & Threshold of feeling & 130 \\
\hline \multirow[t]{9}{*}{ VERY LOUD } & Elevaled train & 120 \\
\hline & Thunder & \\
\hline & Subway train & 110 \\
\hline & Nearty riveter & \\
\hline & Noisy induarrial phent & 100 \\
\hline & Orive with ub machines & 90 \\
\hline & Loud street noise & \\
\hline & Noisy oftice & $\infty$ \\
\hline & Kecuum cleaner & \\
\hline \multirow[t]{3}{*}{ LOUD } & Averafe sereer noise & $\pi$ \\
\hline & Average oftioe & $\infty$ \\
\hline & Congested departmem zore & \\
\hline \multirow[t]{4}{*}{ MODERATE } & Modente restaunnt chatrer & 50 \\
\hline & Average 2-person conversation & \\
\hline & School chasuroom & $\infty$ \\
\hline & Privale of irice & \\
\hline \multirow[t]{2}{*}{ FAINT } & Bedroom & 30 \\
\hline & Ruading loves & $\mathbf{D}$ \\
\hline \multirow[t]{2}{*}{ VERY FAINT } & Normal breathing & D \\
\hline & Thrathold of audibiliny & 0 \\
\hline
\end{tabular}




\subsubsection{Auditory Privacy}

Two kinds of privacy have been identified. Normal privacy- a condition where speech from adjacent locations can be understood if one listened intently, but is not loud enough to cause a disturbance. Confidential privacy precludes the understanding of phrases and/or sentences in adjoining locations.

The preferred method of assessing speech privacy is the Articulation Index (AI). This is a measure of the percentage of sentences that can be understood from a person speaking in a normal conversational tone. The index is based upon signal-tonoise differences in decibels, weighted in terms of the relative contribution of each one-third octave band, to speech intelligibility. As the speech level drops toward the noise level, the AI value approaches zero;as the speech level arises above the noise, the AI nears 1.0. (See following figure.)

Tables 9 and 10 provide recommendations for acoustical performance of selected office activities.

Table 9. Types of Offices Applicable to Articulation Index Ranges (21)

$\begin{array}{cl}\text { I } 00.00- & \text { Confidential Privacy. } \\ 0.05 & \text { Speech cannot be followed } \\ & \text { with understanding. }\end{array}$

II $\quad 0.05-\quad$ Normal Privacy.

0.20 Speech may be understood with careful listening attention but should not interfere with the ability to concentrate on other work.

II 0.20- Marginal Privacy. Provides a relatively quiet office environment when privacy is not of supreme importance.

IV $\quad 0.35-$

0.50

V $\quad 0.50-$

Fair communication, no privacy typical, relatively noisy environment. Does not promote maximum productivity.

Good to excellent communication, no privacy. a) Closed offices with STC of 40-45.

b) Open plan, or office landscape environment.

a) Closed offices with STC of 35-40.

b) Open-plan environmental.

Open-plan environment

Not normally an office feature. Usually found only in conference rooms and auditoriums. 
Table 10. Speech Interference Levels (SIL) and Noise Criteria (NC) Recommended for Rooms (22)

\begin{tabular}{|c|c|c|}
\hline \multirow[b]{2}{*}{ Type of room } & \multicolumn{2}{|c|}{$\begin{array}{l}\text { Maximum permissible level } \\
\text { (measured in vacant rooms) }\end{array}$} \\
\hline & SIL & MC \\
\hline Secretarial offices. Iyping & 60 & $50-55$ \\
\hline Coliseum for sports only (amplification) & 55 & 50 \\
\hline Small private office & 45 & $30-35$ \\
\hline Conference room for 20 & 35 & 30 \\
\hline Movie theater & 35 & 30 \\
\hline Conference room for 50 & 30 & $20-30$ \\
\hline Theaters for drama, 500 seats (no amplification) & 30 & $20-25$ \\
\hline Homes, sleeping areas & 30 & $20-25$ \\
\hline Assembly halis (no amplification) & 30 & \\
\hline Schoolrooms & 30 & 25 \\
\hline Concert halls (no amplification) & 25 & $15-20$ \\
\hline
\end{tabular}




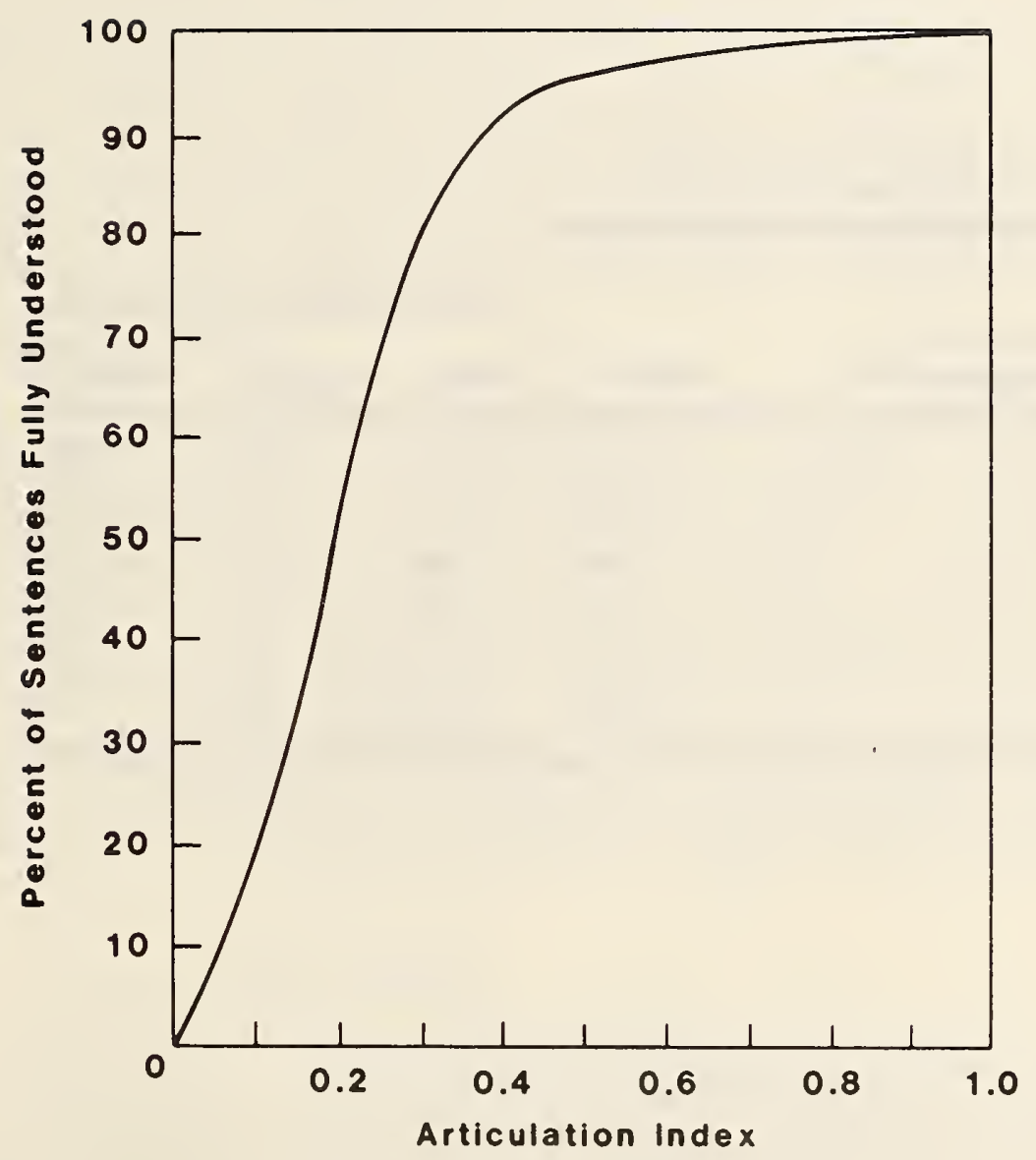

Figure 7. Approximate relation between AI and Speech Intelligibility (2l) 
Another means of evaluating environmental noise, is the speech Interference Level (SIL). These levels constitute the arithmetic average of the sound pressure levels of noise in the three octave bands centered on the frequencies of 500, 1000 and 2000 hertz, based upon complaints of office workers in conducting speech communication. Table 11 demonstrates the relationship between sound level and distance in conducting conversations.

Table 11. Conversations and Speech Interference Levels

\begin{tabular}{|c|c|c|c|c|}
\hline \multirow[b]{2}{*}{$\begin{array}{l}\text { Distance between } \\
\text { talker and listener ( } \mathrm{m} \text { ) }\end{array}$} & \multirow[b]{2}{*}{ Normal } & \multicolumn{3}{|c|}{ Speoch-Interterence lovel (de) } \\
\hline & & Aoked & Vory Loud & Shouting \\
\hline 0.5 & 71 & 77 & 83 & 89 \\
\hline $1: 0$ & 65 & 71 & 77 & 83 \\
\hline 2.0 & 59 & 65 & 71 & 77 \\
\hline 3.0 & 55 & 61 & 67 & 73 \\
\hline 4.0 & 53 & 59 & 65 & $? 1$ \\
\hline 5.0 & 51 & 57 & 63 & 69 \\
\hline 6.0 & 49 & 55 & 61 & 67 \\
\hline 12.0 & 43 & 49 & 55 & 61 \\
\hline
\end{tabular}




\section{4 .2 Sound}

Sound is a form of energy produced by minute pressure fluctuations in the air, generated by vibrating surfaces and aerodynamic turbulence. Most solid objects can potentially produce sound, as does air, when it interacts with structures which change its path, e.g. HVAC ducts.

Sound waves are transmitted directly and indirectly. In direct sound, waves are neither deflected by reflective surfaces, nor absorbed by porous ones. The sound pressure levels of direct sound decreases proportionally with increased distance from the sound source. Indirect sound occurs when the sound source is reflected from hard surfaces such as walls, ceilings or floors. Furnishings, equipment and room features such as light fixtures, can also create sound reflections. Sound is affected by room shape, volume, and the characteristics of the interior surfaces.

\subsubsection{Sound Transmission}

There are two types of sound transmission: airborne and structure-borne. Airborne sound originates and is transmitted directly to the person, e.g. conversation, machine noise. structure-borne sound is produced when an object impacts against a structural element of a building, e.g. footsteps. The sound energy is transmitted in proportion to the mass and rigidity of the structure. A major acoustical problem in many buildings is the presence of flanking (indirect) sound paths, which permit sound to travel throughout many areas of a building. Interior elements such as HVAC ducts, continuous walls between floors, uncaulked junctures of ceilings and partitions can all produce such sound paths.

\subsubsection{Noise Criteria (NC) Curves}

These curves were also developed from "complaint data" from questionnaire surveys. They are intended to specify the maximum noise levels permissible in an environment without eliciting too many complaints. These curves are an indicator of the ability to perform assigned work. The survey responses are then correlated with physical acoustical measurements to specify the acoustical properties of spaces required to perform given activities. Figure 8 illustrates the NC curves. 


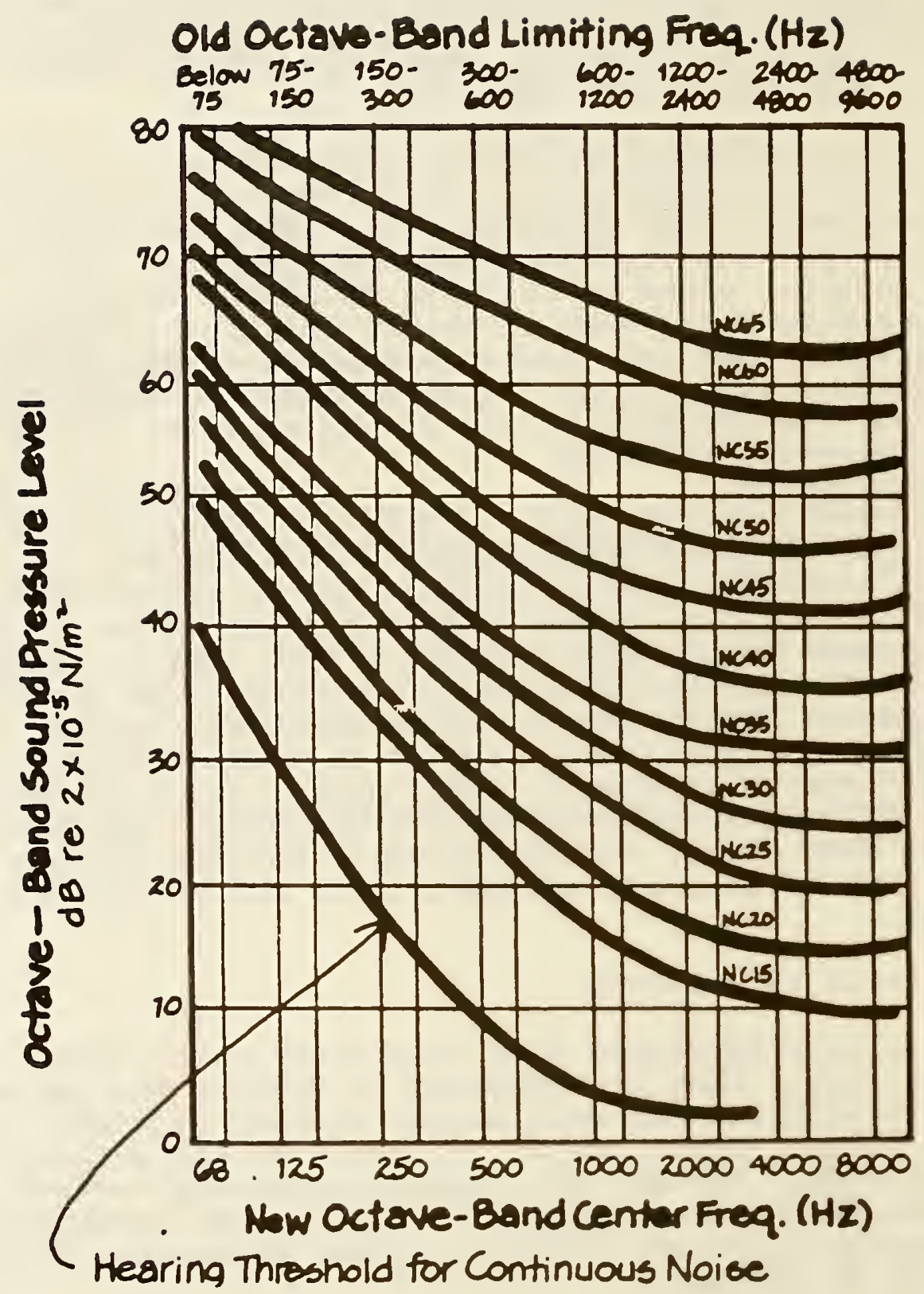

Figure 8. Noise Criteria (NC) Curves 


\subsection{POSSIBLE PROBLEM AREAS}

The solutions being employed to cope with several of the design problems posed by office automation have important implications for acoustical design. For example:

1. Expanded utility chases are placed above a lowered ceiling or beneath a raised floor. Chases provide excellent noise passages, and flanking paths for conversations meant to be private.

2. Supplementary air conditioning units placed above lowered ceilings can introduce undesirable noise sources into the office.

3. Elevated lightweight floors provide excellent sound and vibration radiating diaphragms (i.e. sounding boards) for equipment, machinery and footsteps noises.

4. The mechanization of office tasks results in a proliferation of noisy equipment such as copiers and printers.

5. Open-office planning exposes the office workforce to more noise.

6. Automation of activities has exposed noise sensitive tasks such as planning to noise intrusions.

7. Placement of localized air conditioning units to

dissipate heat load of new equipment.

8. Operation of office machines in open environment when privacy is essential.

9. Planning the introduction of new equipment with minimum disruption.

10. Telephone rings not readily identified with particular phones.

\subsection{TYPICAL SOLUTIONS}

\subsubsection{Barriers}

Sound barriers reduce or eliminate noise by reflecting or resisting its transmission. The mass of the material used as a barrier determines its effectiveness, the greater the mass, the better the performance. The performance of a wall, for example, is measured in decibels (dB). The measurement is referred to as the sound transmission loss (STL). The sound transmission loss class (STC) is a rating scheme using a single number to rate its performance. Typical fixed barrier materials in buildings are sheet rock, gypsum board, concrete, brick or wood. Partitions used in open-offices are movable barriers.

\subsubsection{Isolation of Surfaces}

This is a means of preventing vibration transmissions from one space to an adjoining one. One method of achieving this is to place rubber, compressed fiberglass or springs between surfaces. The isolation of a surface receiving impact, such as a floor or 
ceiling, from the structure, will restrict the transmission of sound.

\subsubsection{Sound Absorption}

The surface treatment of a space is the single most important determinant of its acoustical properties. Sound is absorbed by using porous materials to dissipate or absorb acoustical energy, e.g. fiberglass, cloth. The sound absorption coefficient is a measure of the sound absorbed by a surface; hard materials such as concrete and steel have low values, while carpeting has a relatively high value (the scale ranges from 0 to 1.0 ). Materials used to absorb sound have a low mass, and therefore are not effective in preventing sound transmission to adjoining spaces.

\subsubsection{Vibration Damping}

This is used to prevent a material or object from resonating to a primary source, thereby acting as a secondary noise source, e.g. an air conditioning unit mounted in a window frame. Windows, walls, and machine components are potential problem areas. Rubber or vinyl is typically laminated to the surface of the noise producing element to avoid this problem.

\subsubsection{Isolation of Noise Source}

An effective means of controlling unwanted sound is to isolate and/or "sound treat" the source of noise. For example, duplicating and printing equipment should be located remote from activities requiring quiet. The room should be acoustically treated if noise-sensitive activities are nearby.

4.6.6 Hasking Sound- Background noise is frequently used in open-space designs as a means of securing speech privacy. This "white noise" is generally introduced by means of ceiling speakers. This approach is not recommended because unwanted sound (noise) is not an optimum design solution.

4.6.7 Ceiling Treatment- Those which reflect sound are apt to be the major acoustical problem in an open office. If a high percentage of the ceiling is devoted to light fixtures, major reflection problems are encountered. Ideally, a flat ceiling in properly designed spaces should absorb from 90 to 95 percent of the sound reaching it.

4.6.8 Distance- Individuals and working groups should be clustered (or separated) based on their needs for quiet, and their requirement to interact with one another.

4.6.9 Configuration- Panels and screens can be used to cut off horizontal paths of sound, while providing a degree of acoustic and visual privacy to individuals and/or working groups. 
4.6.10 Floors- Carpeting provides an efficient means of eliminating impact sounds on floors caused by people, equipment and furniture movement. They also have very desirable sound absorption properties.

\subsection{ACOUSTICAL DESIGN PROCESS}

A system has been developed which employs a computer program for the acoustical design of open-plan offices; it is called OPLAN. It is intended to provide an estimate of speech privacy for a given workstation. The program takes into account all paths by which sound travels from one location to another. Data are collected on the following: "anticipated speech effort, source/receiver distance, screen locations and dimensions, materials, background noise levels, and the degree of privacy required." Figure 9 summarizes the approach developed. 


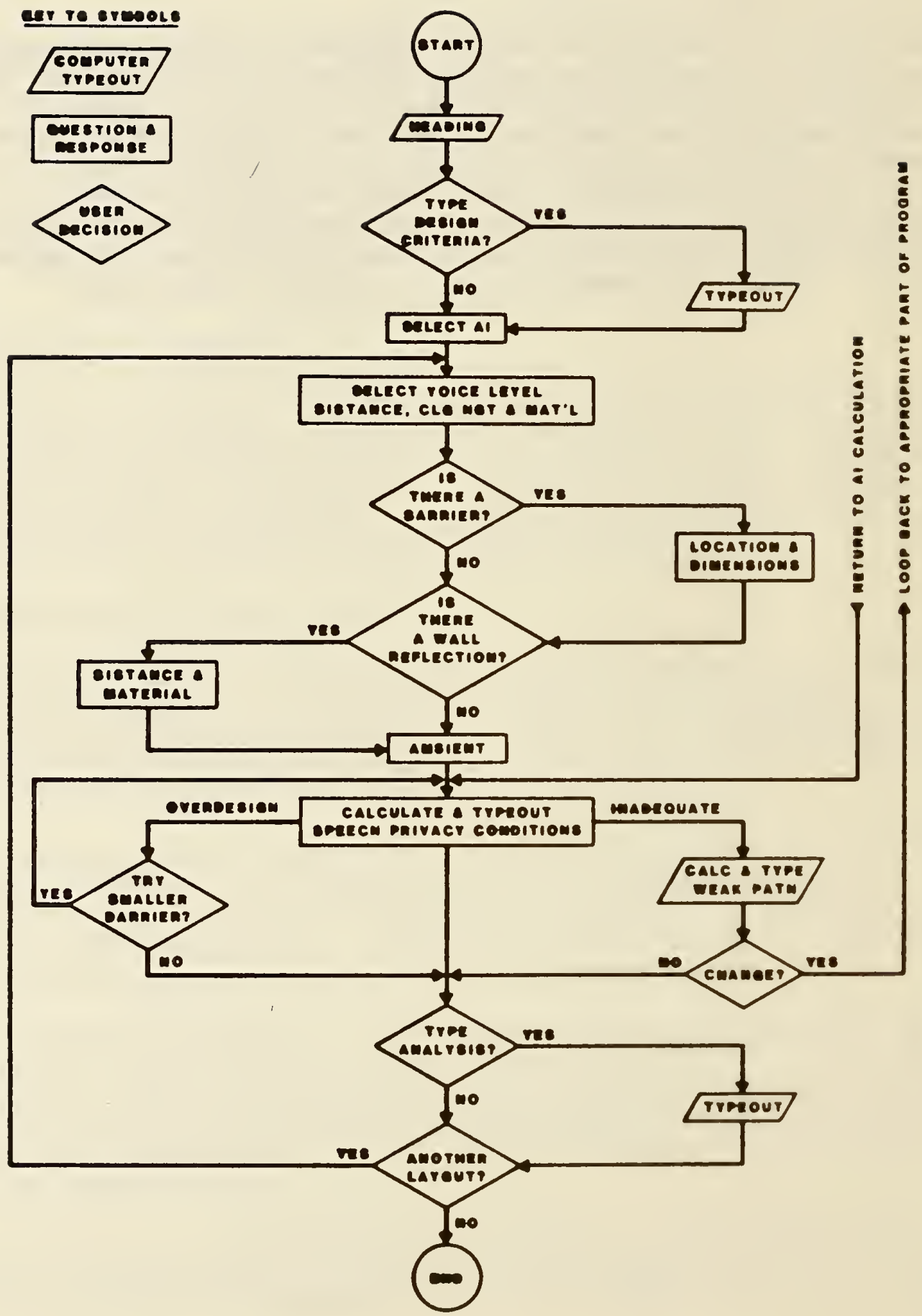

Figure 9. Simplified block diagram of OPLAN program (24) 
NOISE CONTROL

1. The outer shell of the building should provide adequate protection from outdoor noise sources; control of fenestration is particularly important.

2. HVAC and plumbing equipment should be selected, installed and operated to minimize noise; ancillary equipment should not be noisy.

3. Automated equipment should be as quiet as feasible; vibration isolation from floor and furnishings should be considered.

4. Sound absorption properties of ceilings, floors, and panels are a major design concern.

ACOUSTICAL PRIVACY

1. Spaces for confidential meetings should be available in open offices.

2. Adequate separation is required between workstations to ensure proper acoustical comfort.

3. Partitions should be selected and placed in accordance with noise reduction needs.

4. Flanking paths should be avoided between private spaces and the general workfloor; special attention should be given to lowered ceilings, raised floors, and utility chases. 5. Consider using natural sound from HVAC to substitute for masking noise.

\section{VIBRATION CONTROL}

1. Plumbing, HVAC and other mechanical equipment should be vibration isolated.

2. Noisy automation equipment should be vibration isolated.

3. Raised floors should be designed with high vibration damping features.

4. Emergency power generators should be located, installed and used to avoid vibration and noise problems. 


\section{SPACE DESIGN}

1. Individuals and working groups should be clustered in accordance with acoustical requirements, e.g. privacy, freedom from noise intrusions.

2. Noise producing equipment should be grouped and separated from general office activities when feasible.

3. Panels and screens should be used to intercept horizontal sound paths.

4. Ceilings with a high percentage of light fixture area can result in noise reflection problems. 


\subsection{FUNCTIONS}

The primary purpose of lighting is to facilitate the performance of visual office tasks. It provides directional and locational information and is an integral component of security and safety systems. Lighting can create an environment with variety, visual interest and pleasing contrasts, offsetting the institutional appearance of offices.

Effective lighting requires a consideration of the entire environment. A balance of contrasts is needed to prevent visual discomfort and fatigue. The light generated by the VDT, the task area adjacent to the screen, the keyboard area and the surround lighting are also important. Visual problems can occur when the eye must continually adjust to markedly different lighting characteristics, and a variety of contrasts associated with an activity such as reading paper copy while monitoring a VDT screen.

The quality and quantity of light are critical features in automated office design. Proper lighting also depends upon the activities performed, the people doing the work, and organizational requirements. It must take into account traditional building elements such as ceiling heights, wall and floor materials and treatments, room area, adjacent spaces, colurns and various equipment and systems. The integration of lighting with other systems, and flexibility are of special importance in the automated office. Since lighting systems are a major energy user, this feature merits detailed attention. Uniform lighting levels for the most visually demanding task are no longer an acceptable approach to lighting design.

\subsection{BACKGROUND}

Since most office tasks are predominantly visual, it is not surprising that lighting conditions are prominently mentioned in surveys of office workers (25). The use of VDT's in offices has increased the importance of lighting because most lighting systems are designed for desktop paper-based activities, with different visual requirements than those required by VDT tasks. For the foreseeable future most workstations will have to accommodate a combination of both types of activities. Individual control of lighting is an important factor as is the mix of task and ambient lighting.

Office environments are generally designed under the assumption that the worker will perform tasks requiring the line-of-sight to be depressed approximately 20-40 degrees from the horizontal. The design of many VDT's requires line-of-site to be at or near horizontal. This elevation brings the operator's fixation point closer to ceiling luminaires, resulting in a greater possibility of discomfort glare. Exposure to large luminance differences 
between the VDT screen and some other part of the visual surround, such as a window or luminaire, increases the possibility of discomfort glare. Glare reflected from surfaces of the VDT and ceiling reflections reduce the visibility of the display by reducing the physical contrast between the characters and the screen background.

\subsection{CRITERIA}

* Lighting should reinforce the architectural intent of the building, e.g. provide a connection with the outside and between spaces; create a sense of intimacy and the delineation of activity spaces.

* The quality and quantity of lighting should support the visual needs of those performing VDT and/or paper-based tasks.

* Levels should be consistent with the tasks performed

* Lighting should not produce glare.

* Extreme brightness contrasts should be avoided.

* Individual control of task and area lighting should be available.

* Systems should provide flexibility appropriate for organizational needs, including expansion.

* Systems should not be wasteful of energy.

* Systems should be properly integrated with other building systems - e.g. ceiling, wiring, automation, safety.

* Lighting should provide proper margins for safety and security activities.

* Systems should be designed for ready maintenance.

* Daylight should be appropriately integrated into the overall lighting system design.

* Two lighting level systems should be considered for flexibility. 
Table 12. Lighting Recommendations- Illuminating Engineering Society (IES) (26)

\begin{tabular}{|c|c|c|c|c|}
\hline \multirow{2}{*}{ Type of Activity } & \multirow{2}{*}{$\begin{array}{l}\text { Illurinance } \\
\text { Category }\end{array}$} & \multicolumn{2}{|c|}{ Ranges of Illuminances } & \multirow{2}{*}{$\begin{array}{r}\text { Reference } \\
\text { Work-Plane }\end{array}$} \\
\hline & & Lux & Footcandles & \\
\hline $\begin{array}{l}\text { Public spaces with } \\
\text { dark surroundings }\end{array}$ & $\mathbf{A}$ & $20-30-50$ & $2-3-5$ & \multirow{4}{*}{$\begin{array}{l}\text { General } \\
\text { l1ghting } \\
\text { throughout } \\
\text { spaces }\end{array}$} \\
\hline $\begin{array}{l}\text { S1mple orlentation for } \\
\text { short temporary visits }\end{array}$ & B & $50-75-100$ & $5-7 \cdot 5-10$ & \\
\hline $\begin{array}{l}\text { Work1ng space where } \\
\text { v1sual tasks are only } \\
\text { occasionally performed }\end{array}$ & C & $100-150-200$ & $10-15-20$ & \\
\hline $\begin{array}{l}\text { Performance of visual } \\
\text { tasks of high contrast } \\
\text { or lerge size }\end{array}$ & D & $200-300-500$ & $20-30-50$ & \\
\hline $\begin{array}{l}\text { Performance of visual } \\
\text { tasks of vedlum } \\
\text { contrast or small size }\end{array}$ & $\mathbf{E}$ & $500-750-1000$ & $50-75-100$ & \multirow[t]{2}{*}{$\begin{array}{l}\text { Illuninance } \\
\text { on task }\end{array}$} \\
\hline $\begin{array}{l}\text { Performance of visual } \\
\text { tasks of low contrast } \\
\text { or emall size }\end{array}$ & $\mathbf{F}$ & $1000-1500-2000$ & $100-150-200$ & \\
\hline $\begin{array}{l}\text { Performance of visual } \\
\text { tasks of low contrast } \\
\text { and very emall olze over } \\
\text { a prolonged perlod }\end{array}$ & G & $2000-3000-5000$ & $200-300-500$ & \multirow{3}{*}{$\begin{array}{l}\text { Illuminance } \\
\text { on task, } \\
\text { obtalned by } \\
\text { a comblnation } \\
\text { of general } \\
\text { and local } \\
\text { (supplemen- } \\
\text { tary } \\
\text { l1ght1ng) }\end{array}$} \\
\hline $\begin{array}{l}\text { Yerformance of very } \\
\text { prolonged and exacting } \\
\text { vlsual tasks }\end{array}$ & $\mathbf{H}$ & $5000-7500-10000$ & $500-750-1000$ & \\
\hline $\begin{array}{l}\text { Performance of very } \\
\text { epecial visual taks of } \\
\text { extremely low contrast } \\
\text { and small size }\end{array}$ & $\mathbf{I}$ & $10000-15000-20000$ & $1000-1500-2000$ & \\
\hline
\end{tabular}




\subsection{OVERVIEW OF BASIC ISSUES}

Office lighting is achieved by a combination of natural and artificial lighting. Daylight is a varied, changing source of color and intensity, influenced by atmospheric conditions such as cloud cover and pollution, the position of the sun, time of day, reflection of building materials, trees and grass.

The characteristics of the surrounding environment must also be carefully controlled to ensure the visual comfort of VDT operators. Walls and other surfaces should have reflectance values of less than 50 percent to accomplish this. All desk and work surfaces should be comprised of matte, or low glare nonreflective surfaces, and medium tone colors. They should not be black or white, or exhibit sharp color contrasts or reflective surfaces, to minimize visual irritation. VDT's should be positioned so they are not parallel to each other. This avoids the possibility of light from screens reflecting on one other. A test to ensure that the major problems have been eliminated is to move a mirror across the front surface of the VDT screen. This will reveal bright sources of light.

\subsubsection{Artificial Light Sources}

The most common sources are incandescent lamps, gaseous discharge lamps such as fluorescent, and high intensity discharge (HID) lamps.

\subsubsection{Incandescent Lamps}

Light is produced when a wire of tungsten filament is heated to intense brightness by the flow of electric current. Although all spectrum colors are present, the light tends to be strong in the red, orange and yellow wavelengths. Colors in the "warm" spectral range are therefore strengthened when illuminated by incandescent sources, while "cool" colors such as blue and green are weakened. The efficiency of these lamps is low - i.e. the amount of light produced by a given amount of energy. Efficiency is directly related to filament temperature and, for incandescent lamps, the most efficient ones have the shortest lives. Figure 10 below depicts several types of lamps.

Figure 10 is an illustration of the common types of lamps in use for lighting offices. 


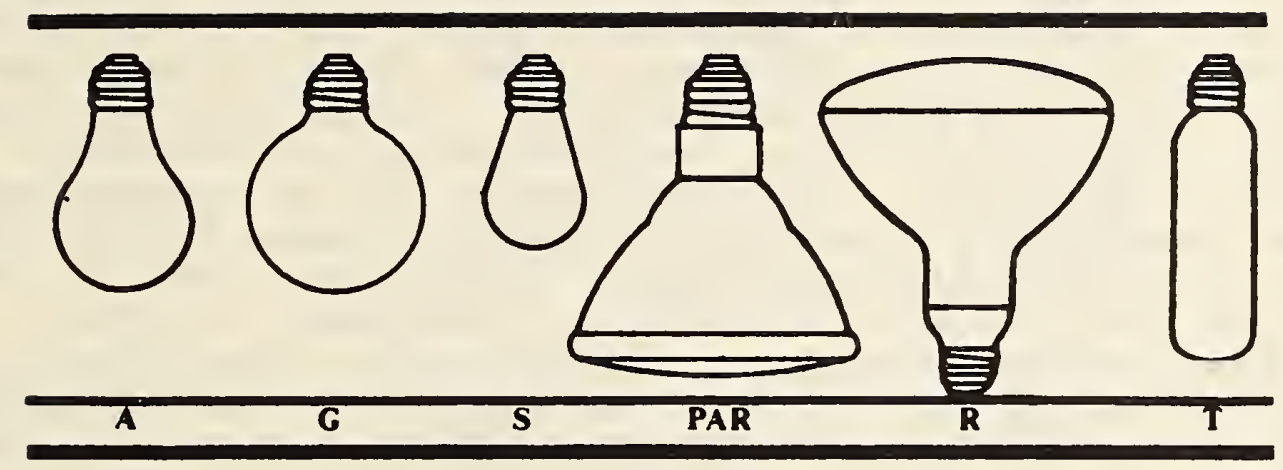

A- General service lamp, G- Decorative lamp, S- Decorative lamp, PAR- Lamp used for directional purposes, e.g. downlight, used with reflective or baffled fixtures in recessed, surface or track lighting. R- A type of PAR downlight with a wide beam spread. T- A tungsten-halogen lamp, designed for long life and high intensity output; used for general lighting in medium-to-high ceiling areas.

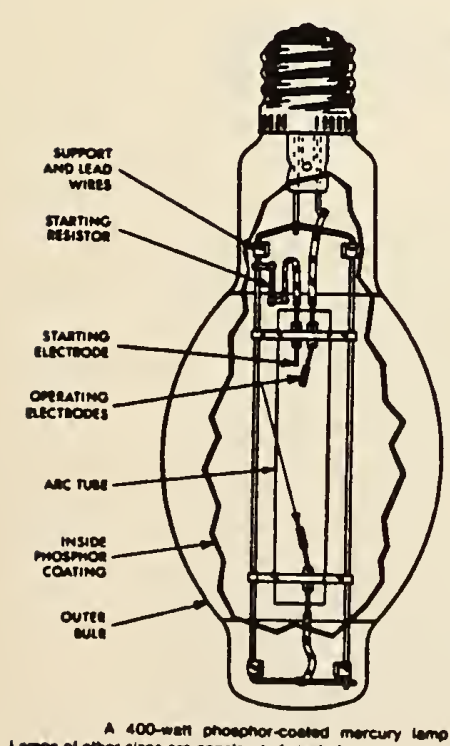

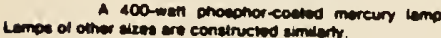

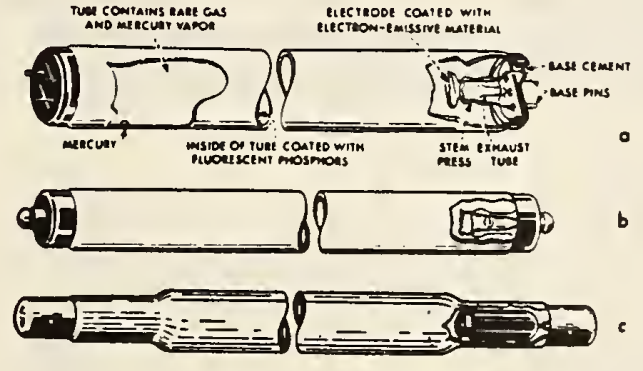

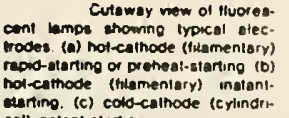

cal) mstent-kiarting

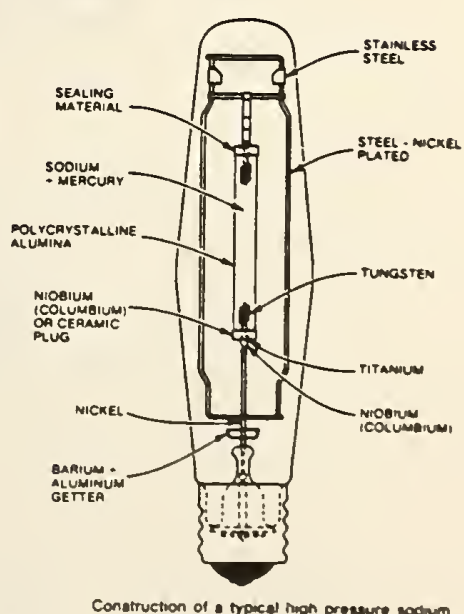

Figure 10. Common Types of Lamps 


\subsubsection{Fluorescent Lamps}

Fluorescent lighting occurs when a phospher powder lining along the inner tube wall of the lamp, fluoresces or glows. The powder is activated by ultraviolet radiation emitted from low pressure mercury vapor in the tube. They are higher in efficiency than incandescent lamps and produce less heat. Furthermore they last from 20 to 25 times longer than incandescents, and therefore require less maintenance. Lamp performance can be overestimated if measured immediately after installation, because they produce more light during the first 100 hours of operation than in long time use. The lamps should therefore be "seasoned" for this length of time before occupancy. This procedure also identifies faulty ballasts and lamps because when defective, early problems are likely. The color rendition properties of fluorescent lamps depend upon the particular coatings and gases used in design.

\subsubsection{High Intensity Discharge (HID) Lamps}

They produce light through the gaseous discharge or vaporization of atoms of metallic elements contained in arc tubes. Vaporization causes the atoms to emit large amounts of electromagnetic energy within the visible range. An arc of electricity generates heat and pressure much higher than in the ordinary fluorescent lamp. Mercury, sodium and metal halide lamps are characterized by wide light distribution, wattage efficiency and low energy consumption and maintenance costs. Among the shortcomings of HID lamps are the following:

(1) High light reduction over the life of the lamp (up to 50 percent)

(2) A limited number of colors are available

(3) There are sometimes problems with color stability and the standardization of particular colors. 
Table 13. Lamp Selection Guide (28)

\begin{tabular}{|c|c|c|c|c|c|c|c|}
\hline 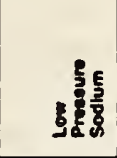 & . & 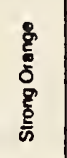 & 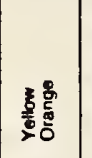 & 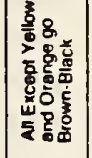 & $\nsubseteq$ & \$ & 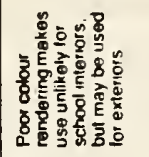 \\
\hline 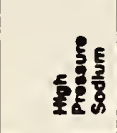 & \&్లి & 旁 & 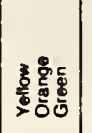 & 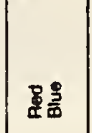 & $\stackrel{\mathbb{Z}}{2}$ & 気 & 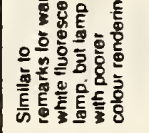 \\
\hline & 贾 & 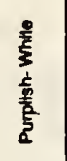 & 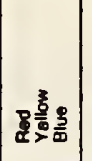 & छ్ & ฆ & 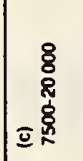 & 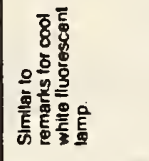 \\
\hline & 8 & 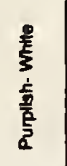 & 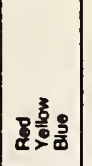 & $\underline{\delta}$ & 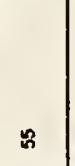 & 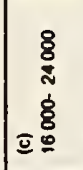 & 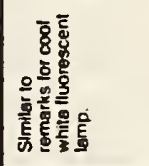 \\
\hline & 8 & $\begin{array}{l}\frac{8}{5} \\
\frac{1}{2} \\
\frac{1}{2} \\
\frac{1}{2}\end{array}$ & 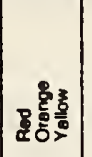 & $\frac{3}{4}$ & $\&$ & 悹 & 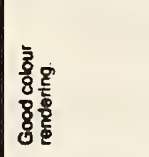 \\
\hline & 8 & $\begin{array}{l}\frac{0}{5} \\
\frac{5}{5} \\
\frac{5}{5} \\
\frac{5}{2} \\
\frac{5}{2}\end{array}$ & 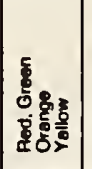 & $\frac{3}{9}$ & 范 & $\begin{array}{r}8 \\
8 \\
8 \\
8 \\
8 \\
0\end{array}$ & 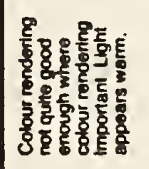 \\
\hline & $\frac{8}{7}$ & $\begin{array}{l}\frac{0}{5} \\
\frac{1}{2} \\
\frac{1}{2} \\
\frac{1}{2}\end{array}$ & $\mid$ & 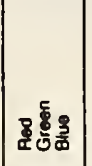 & 8 & $\begin{array}{r}8 \\
8 \\
8 \\
6 \\
\text { s. } \\
\approx\end{array}$ & 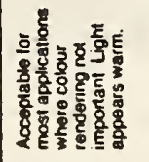 \\
\hline & ६ & $\frac{8}{\xi}$ & 㕍要 & 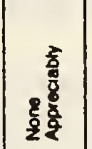 & 葛 & 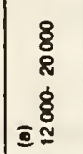 & 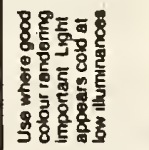 \\
\hline & 条 & $\dot{\xi}$ & 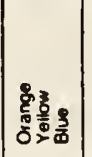 & $\not$ & 8 & 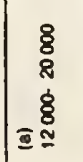 & 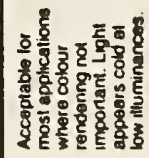 \\
\hline 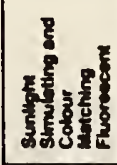 & 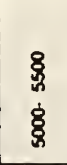 & 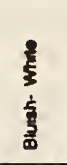 & 潼要 & 意 & $\tilde{\tilde{s}}$ & 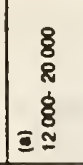 & 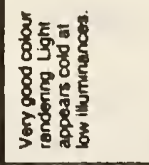 \\
\hline & 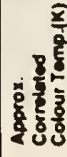 & 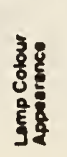 & 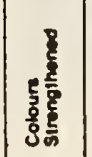 & 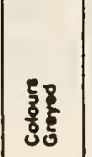 & 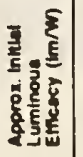 & 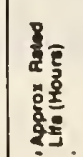 & $I^{\alpha}$ \\
\hline
\end{tabular}

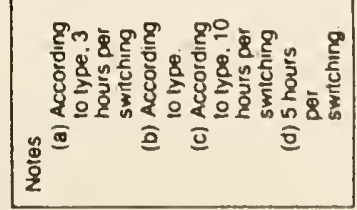




\subsubsection{Ballasts}

A ballast is used to limit the flow of current through fluorescent and HID lamps. Without it, current increases to a level which will quickly destroy the lamp. The ballast provides a voltage that is sufficiently height to start and maintain the arc discharge in the lamps. Special ballasts are now available which conserve energy and permit the dimming of the lamps. Ballast hum can be a side effect of HID and fluorescent lamps, especially as they age.

\subsubsection{Fixtures}

Incandescent housings are sometimes movable (table, pole, track or floor lamp), or fixed (recessed, surface-mounted, ceiling hung). The quality and distribution of light depend on the type of bulb used, the diameter of the fixture aperture, the interior shape and surface finish of the fixture, the distance of the lamp from the aperture, and the distance of the fixture from the surface to be illuminated. Interior fixture surfaces are often coated with a color and/or a special finish to produce given lighting effects.

Offices today are predominantly lighted by fluorescent lighting systems. Housings for these systems are ceiling recessed, surface-mounted, or pendant ceiling-hung. The quantity of light produced is primarily dependent on the number and wattage of lamps used in each fixture. The quality of lighting is influenced by the lens and/or louver system employed to distribute the light. A major determinant of lighting quality is the "glare producing" potential of a given system.

\subsubsection{Lighting Fixture Rating Schemes}

\subsubsection{Visual Comfort Probability (VCP)}

This is a rating of the likelihood that people using a given system would not be bothered by direct glare from a given fixture design. For example, a VCP of 70 means that 70 of of those workers seated in the worst location in a given space would not be bothered by direct glare from a lighting system composed of the type of fixture whose VCP is under evaluation.

\subsubsection{Coefficient of Utilization (CU)}

It is a measure of the efficiency of a fixture in a specified space, i.e. the percentage of the light produced by a lamp which ultimately reaches the work area. For example, a fixture with a rating of .50 delivers 50 percent of the lamp's output to the work plane. 
The VCP and CU of any given fixture depend upon such factors as room configuration, reflectivity of surfaces, distance from the floor, and the spacing between fixtures.

\subsection{POSSIBLE PROBLEM AREAS}

* Lighting systems at workstations must accommodate VDT and paper-based tasks

* The line-of-sight of the VDT screen is near the horizontal, resulting in a greater likelihood of glare from a fixture in direct view.

* Reflected glare can occur from reflections on the surface of the VDT, from windows, fixtures, people, and other VDT's. * Extreme visual contrasts in the visual field, created from VDT, the workspace, general surrounds, windows, etc cause visual discomfort.

* The placement of fixtures may be inappropriate for the tasks being performed - e.g. shadows, glare.

* Indirect HID lighting can produce "hot spots" on the ceiling; they also might cause surface finish problems.. * Color rendition of HID sources can result in aesthetic problems, especially when materials and furnishings are not carefully selected with regard to color characteristics. * Color stability and uniformity are sometimes a problem with HID sources.

* Highly reflective materials can be sources of reflected glare.

* Black and white "colors" can contribute to undesirable visual contrasts in the work setting.

* Dirt and dust on fixtures and lamps can substantially reduce light output; scheduled maintenance is important. * Fluorescent task lamps can emit electronic noise, affecting devices nearby.

\subsection{TYPICAL APPROACHES USED IN LIGHTING DESIGN}

\subsubsection{Types of Illumination}

Three general approaches are available for office lightinggeneral (ambient), direct, and indirect. Ambient lighting refers to a uniform pattern of lighting throughout a space. This approach is being replaced in many offices by task/ambient systems which are at a relatively low level for movement and circulation purposes (i.e. less demanding visual tasks), and direct lighting on the task to be performed. Direct lighting has the advantage of efficiently using the available light to accomplish a given purpose, but glare and veiling reflections must be avoided. Indirect lighting uses wall and/or ceiling surfaces to reflect light into the workspace, e.g. for ambient lighting, architectural definition. 


\subsubsection{Ambient (General) Lighting}

An ambient system provides uniform illumination across the entire space, at the level required to support the most visually demanding visual task to be performed in the office. The location of particular tasks do not have to be specified because the assumption is that if the lighting level is appropriate for the most demanding task, then it will be sufficient for less difficult ones. The connected power requirements are a function of the overall lighting level and the size of the area served. Ambient lighting can be provided by direct (usually part of the building system) or indirect means.

Ceiling fluorescent systems are usually employed for ambient lighting. The first consideration is to avoid lamp reflection on the face of the VDT. This can be accomplished by using a specular parabolic wedge louver; it has a typical cutoff of 45 degrees, with images from the lamp reflected below the viewing angle.

\subsubsection{Task Lighting}

Lighting is directed to a specific surface or area to provide illumination for visual tasks. Typically, light sources are placed on top of a desk, hung from a panel, placed above a work surface, or under an overhead bookcase.

\subsubsection{Task/Ambient Lighting}

In task/ambient systems, task lighting is treated separately from the general surround (ambient), which is usually at a lower level. Task location must be known in advance to ensure the adequate space planning density, and the particular needs of workstations which house different tasks. Power use is variable because lighting is typically controlled by the user. The overall space will appear non-uniform because of the lack of a standardized lighting scheme. Task lighting systems can be furniture mounted, free standing, or depend on suspended luminaires. The major requirement is to meet the needs of individual users with diverse performance, habits, and visual abilities.

\subsubsection{Indirect Lighting}

Another method employed to illuminate VDT work is indirect lighting, the source is mounted below the ceiling, suspended from the ceiling, or placed in a kiosk. In all instances, the light must be directed toward the ceiling, which acts as a reflector. Rays are directed laterally, resulting in shadow-free lighting with the light source hidden from view, and eliminated as a possible irritant. 

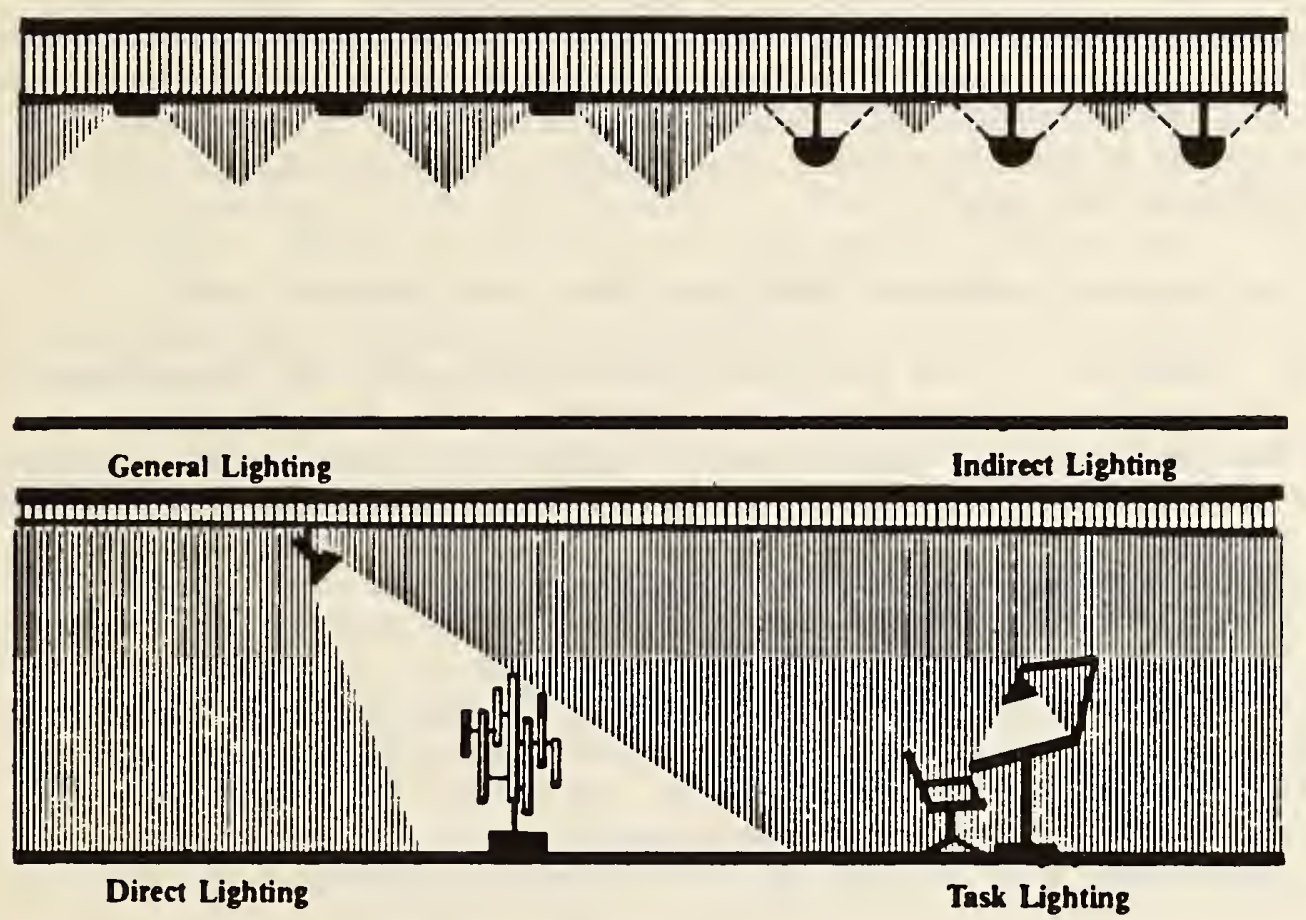

Figure 11. Types of Illumination

Checklist for Evaluating Task/Ambient Systems

1. Lighting should be evenly distributed.

2. Veiling reflections should be minimized.

3. A proper brightness relationship between task and

surround is essential. (No more that 3:1 in the immediate seeing zone, no more than 10:1 in the total zone. 4. Luminaires should be glare free and the light source not visible.

5. Ballast noise should be minimized by using properly mounted, high quality ballasts.

6. Ballast and housing should not radiate excessive heat.

7. Fixtures should be easy to clean and maintain.

Replacement of lamps and ballasts should be an easy task.

8. Fixtures should be properly mounted to prevent vibration.

9. Upward light distribution should minimize shadows, while avoiding "hot spots".

10. Downlights should light the rear of the workstation uniformly. 


\section{$5 \cdot 6.2$ Controls}

Lighting controls provide the operator with the means to adjust the lighting to suit the activity to be performed. They also serve an important energy-saving function. Local lighting controls can activate a small number of fixtures which permit the lights to be readily turned off when not needed. Dimmers also provide the opportunity to adjust the lighting conditions as visual requirements change.

A variety of automated control systems are available; they frequently are dependent on a combination of manual and automated features. For example, time switches are employed in areas not often used. In some configurations, lighting is activated manually and automatically deactivated after a given time, unless manual intervention occurs. Some centralized lighting controls send signals to fixtures over existing circuitry; others use radio frequencies or require new wiring.

Controls are available which work on the principles of motion detection (ultrasonics), body heat (passive infra-red), noise in the human activity range (acoustics), or the interruption of beams transmitted and detected (active infra-red). Carbon dioxide is also used as an occupant sensor. ( Some of these systems are also used for building security purposes.)

Window lighting should be controlled by curtains and blinds which also filter and direct sunlight at reasonable cost. Vertical blinds can be turned and pivoted to redirect sunlight. A fabric $\mathrm{finish}$ is preferable because it diffuses the light, reducing glare. Mylar and plastic filters and screens can be positioned in front of windows like sunshades.

\subsubsection{Maintenance}

Lamps should be replaced before failure because light output declines with use. Replacing lamps early therefore maintains a greater average light output of the entire system. Relamping before failure also lengthens ballast life. Group relamping is best accomplished by changing all lamps at the same time. When personnel, equipment, and lamps are assembled simultaneously, a lamp change is made in a few minutes. In contrast, single lamp replacement can take as long as 30 minutes. A further savings is realized if fixtures are cleaned during scheduled relamping. A periodic cleaning schedule for fixtures and lamps is a requirement, if designed lighting levels are to be maintained over the lifetime of the building. Dust and dirt accumulations can severely limit the efficiency of lighting systems (30).

\subsection{ENVIRONMENTAL CONTEXT}

When a person is operating a VDT, he or she looks not only at the work materials, but peripherally at the work surface, distant 
furniture and walls, windows and the general office area. Sharp contrasts in color and reflective surfaces should be avoided because they may result in visual distractions and/or difficulty in performing required tasks.

Brightnesses within the office space should be controlled. Walls and other surfaces should be diffuse and have a light reflectance value of less than 50\%. Carpeting colors should be muted rather than saturated in color. Desks and work surfaces should be matte, or low-glare non-reflective. The tops of tables and desks should be made of non-specularly reflective material. Neutral color tones are preferred for all furnishings.

\subsubsection{Checklist for Glare Control}

Direct and reflected glare can be limited by means of one or more of the following methods:

* Drapes, shades, and/or blinds over windows should be closed or directionally adjusted, especially during sunlight conditions.

* Terminals should be properly positioned with respect to windows and lighting fixtures, e.g. screens facing away from bright sources.

* Direct lighting fixtures may need to be recessed; baffles or special covers are used to direct light sources downward. * Indirect lighting fixtures should have appropriate lens/louver systems to minimize glare potential. * Avoid specular surfaces on furnishings and equipment. 


\section{ENVIRONMENTAL CONDITIONING SYSTEMS}

\subsection{FUNCTION}

HVAC systems provide for thermal comfort and well-being of office occupants and help maintain the proper environment for support systems operation by regulating (32):

* air temperature

* the temperature of surrounding surfaces

* relative humidity and air motjon

* air quality- e.g. freedom from unwanted odors anà particulate matter

6.2 CRITERIA (33)

* Air temperatures and humidity must accommodate equipment and people.

* Flexible air supply and return systems should be designed to facilitate change and/or expansion.

* Humidity control must accommodate the needs of people and equipment.

* Thermal comfort conditions should follow ASHRAE

recommendations. (See Figure 12.)

* Energy usage is of major importance.

* Control systems should enable area zoning to accommodate equipment and activities.

* Static electricity shoula be minimized.

* Air distribution system should permit change in workstation locations, maintaining thermal comfort anả indoor air quality.

\subsection{OFFICE AUTOMATION DESIGN IMIPLICATIONS}

Automated office have more electronic devices and systems than traditional ones. This technology produces considerable heat which substantially increases building cooling loads, resulting in eguipment outages and occupant complaints. Many devices are humidity sensitive; proper control is essential for the equipment operation and to minimize the detrimental effects of static electricity on workers and electrical equipment. A combination of tight building designs to save energy, and using chemicals in office activities, such as ink for duplicating machines, cleaning chemicals, anả particle board, degrades office air quality. 


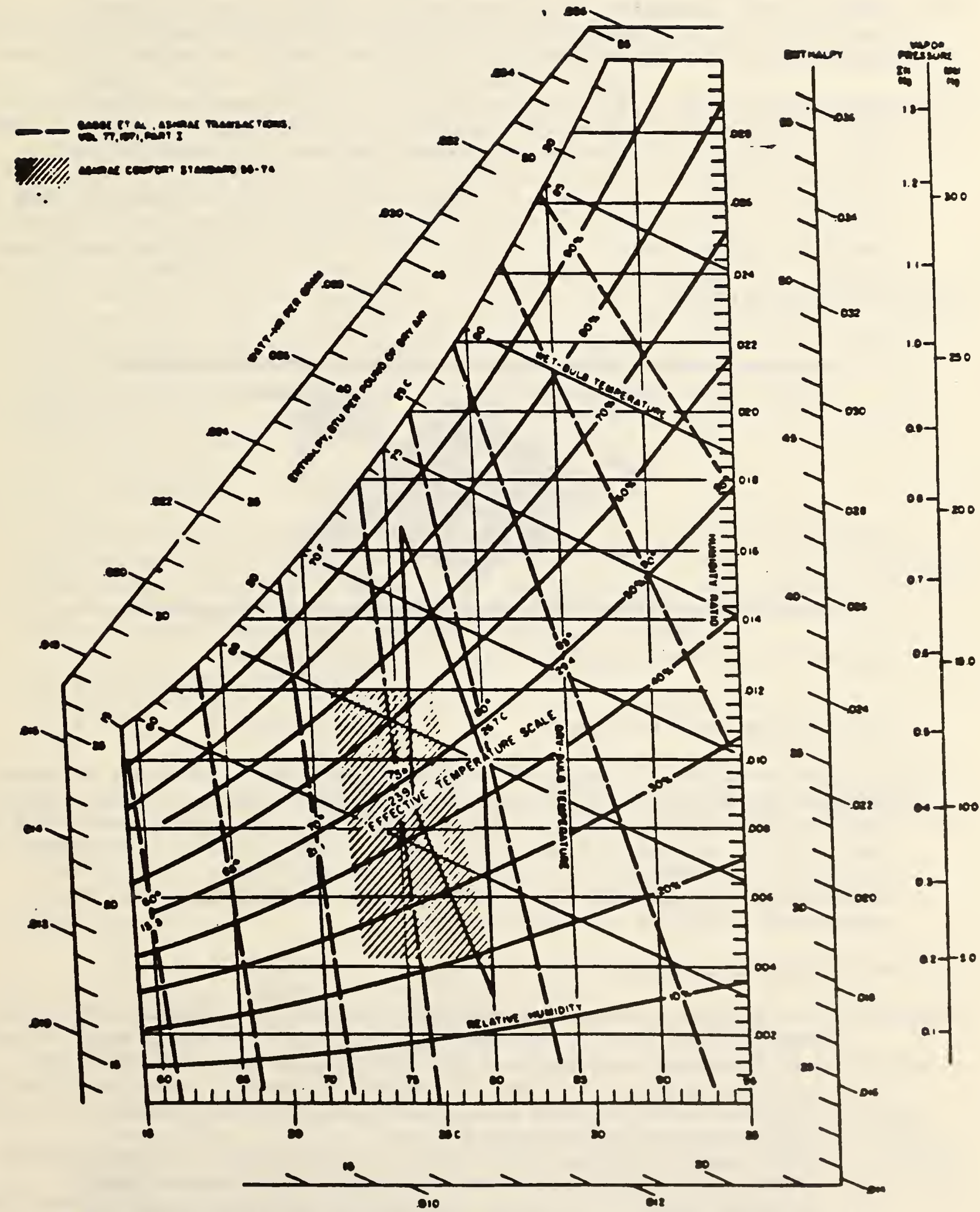

* The envelope applies for 11ghtly clothed, sedentary Individuals in spaces with low alr movement, where the MRT equals alr temperature.

Figure 12. Thermal Comfort Recommendations ASHRAE) 


\subsection{TYPICAL APPROACHES}

Meeting environmental requirements in offices is not easy. Conditions in a space served by a central system nay vary markedly, depending on the distribution of people and electronic equipment.

The conventional system of ceiling air distribution consists of a main trunk and a grid of either flexible or rigid auxiliary feeder ducts. Adequate clearance is needed between the ceiling and structure above the ductwork and access to mechanical equipment in the plenum space. Supply and return air grills in the ceilings must conform to the module of the ceiling system. Newer and proposed systems employ supply and return air through the floor and some deliver air through workstation units with localized control. See following figure.

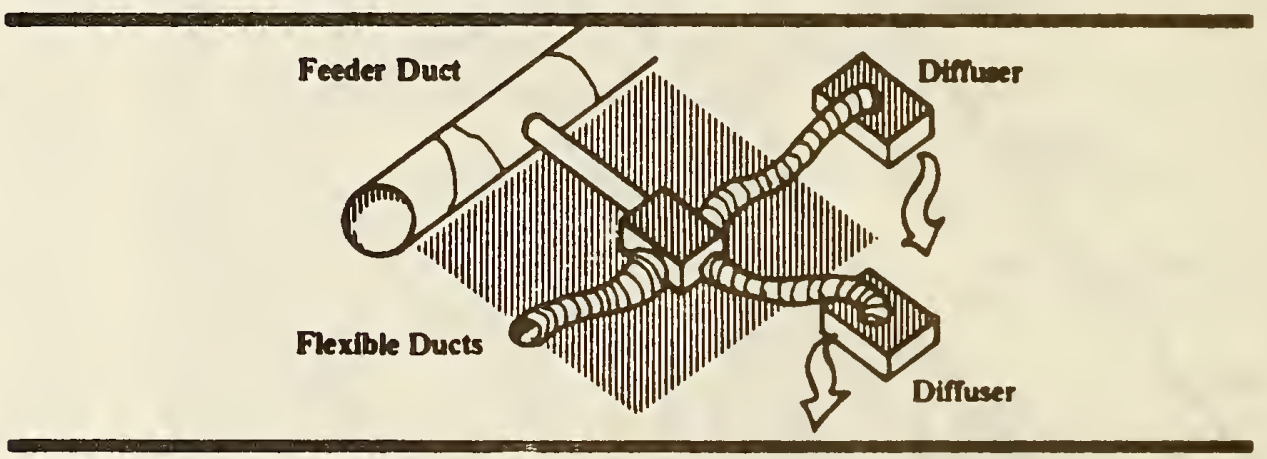

Figure 13 Conventional ceiling air distribution

6.4.1 Existing Buildings

For spaces with clusters of computer equipment, modular ceiling mounted HVAC units can be used to supplement the central system. Localized controls should be available for this equipment so that they can be turned off when not used. Heat-recovery systems from clustered workspaces are applicable in high heat load areas. Packaged air conditioning and humidity control systems can supplement central building systems.

\subsubsection{New Construction}

* Variable air volume systems are used to maintain desired temperature levels by controlling air volume instead of supply air temperature, for energy savings. If air movement is insufficient, fan-power is added. (However, some problems have occurred with regard to air motion and ventilation in occupied spaces with these systems.)

* Thermostats must be carefully positioned in open spaces; out of the path of the return air supply, and at a height

representative of office activities. Separate units are needed in each zone.

* Floor plenums should be considered for HVAC/electrical distribution. 
* Zoning, using individual HVAC systems, can be used to service core areas subjected to concentrated heat loads. They require year round cooling even in northern climates.

* Portions of the building might be occupied on a 24 hour per day basis and merit separate treatment.

* Computer rooms should have localized air conditioning units, which often require backup power supply.

* Ceiling-mounted air cooled systems operating independently of central systems are available. However, installation cost and difficulty in moving them are shortcomings.

\subsection{INTERACTION WITH OTHER SYSTEMS}

\subsubsection{Lighting}

Heat removal and air handling lighting fixtures extract heat before it enters the air conditioning system, thereby reducing the overall heating load. Furniture-integrated lighting systems emit heat directly into the air. Lighting and HVAC system design must be integrated to optimize lighting efficacy and useful heat generation; e.g. systems which recover and/or remove heat from lighting and other devices, thereby saving energy.

\section{5 .2 Acoustics}

Air distribution systems can be designed to produce relatively uniform sound, thereby eliminating the need for the introduction of white noise into office spaces as an aid to privacy. Inconsistency in acoustic quality results from variable air volume and other systems which repeatedly switch from on to off.

\subsection{ENVIRONMENTAL CONTEXT}

\subsubsection{Design Process Issues}

Better planning and coordination is needed for implementing office automation systems. Improved coordination is required among the user, the $A / E$, and the consultants involved in designing the office systems.

Among the issues that require detailed architectural programming attention are:

* Means of achieving proper air distribution with provision for future changes at minimum cost.

* Determining tradeoffs among automation, energy conservation, and thermal comfort requirements at each design stage.

* Responding to a strategic automation plan by the building user.

* Integrating HVAC effectively with other building systems.

* Providing control systems responsive to specific needs of equipment and people, e.g. older workers often require warmer temperatures than younger ones; requirements differ with the physical demands of tasks. 


\subsubsection{Interior Design}

* Window coverings, wall fabrics, floor coverings and adjustment of air flow patterns all influence the thermal comfort of a space. The proper placement and adjustment of diffusers and radiators also contribute to environmental satisfaction.

* Window exposures affects the thermal environment in significant ways. For example, in the winter months, southern exposure can contribute to the heat needed.

* Functions that require similar environmental conditions can be grouped together, focusing mechanical demands appropriately.

\subsubsection{Potential Problem Area - Static Electricity (35)}

Electrostatic discharge is often a major problem in automated offices. Static electricity occurs in two ways, induction charging and contact charging. In induction charging, electrical fields radiate from the surfaces of materials such as styrofoam and cloth. For example, an electrostatic discharge from a shirt sleeve can generate power to destroy the read only memory (ROM) of a computer. In contact charging, two materials make contact with one another and then separate. This action causes one material to strip electrons from the other. This occurs when one walks across a carpet and is shocked when touching a doorknob. The size of a charge depends on the speed, degree of friction, and characteristics of materials into contacting one an other. The higher the electron density of the material, the greater its charge. While voltages of 3,000 are sometimes sufficient to disturb computer circuits, it is not until voltages are in the 10,000 to 15,000 range that people become aware of unpleasant or painful sensations. Table 14 summarizes some effects of static electricity.

\subsubsection{Common Sources of Static Electricity}

* Waxed, painted or varnished surfaces

* Vinyl tile flooring

* Polystyrene (Styrofoam)

* Finished wood or plastic covered desks, chairs

* Electrostatic copiers

* Insufficient humidification 
TABLE 14. Static Electricity Issues

ACTIVITY

$$
\text { VOLTAGE }
$$

$$
10-20 \% \mathrm{RH}
$$

$65-90 \% \mathrm{RH}$

Walking on carpet

35,000

1,500

250

Walking on vinyl floor

12,000

1,200

Picking up polyethelene bag

20,000

1,500

ENDANGERED COMPONENTS

Precision voltage regulator circuits Transistor logic devices

Silicon power transistors

Small signal diodes

$1-1,000$

$1,000-4,000$

$4,000-15,000$

$4,000-15,000$

6.6.3.2 Possible Approaches to Overcome Problem

* Power Grounding

* Humidity control. Humidity in the 40 - 60 percent range is recommended by manufacturers of office automation equipment.

* Carpeting materials should be selected for low static electricity properties.

* Chairs and movable furnishings should have metal, conducting rubber or plastic wheels.

* Conductive anti-static floor mats can be used to bleed static charges to ground, through a wire. The mat must be free of dirt and wax; operators must wear conductive footwear. * Anti-static agents can be sprayed directly on carpet or tile. This increases the rate at which air ions recombine with surface charges by maintaining a thin moisture layer over the area applied.

* An ionizer can be used; it emits both positive and negative electrons which combine with opposite charges on the surfaces of non-conductive materials such as cloth and plastics.

\subsection{AIR QUALITY}

\subsubsection{Background}

Air quality traditionally refers to outdoor air, and is related to levels of specific pollutants designated by the Environmental Protection Agency (EPA) as acceptable for good health. No formal guidelines govern the quality of indoor air. Another difficulty is that air quality is not limited to health issues; odors and humidity levels are important, especially indoors.

For many pollutants, indoor concentrations are four to ten times more than outdoor ones. In computer rooms, printout paper has a high concentration of dust. Microscopic particles are in the air and when humidity is low, the particles often cause itching. 
Pollutants include gases given off from combustion processes, airborne particles resulting from activities such as smoking, and gaseous emissions from materials.

Energy conservation has led to buildings designed to reduce air leakage through cracks and around doors, windows, and other openings. Potential air pathways are blocked and/or minimized by caulking and weather stripping. Added insulation reduces temperature loss through the structure by adding thermal resistance to walls, ceilings and floors.(36)

As buildings are designed for energy efficiency, they are less forgiving environmentally. The capacities of mechanical equipment more closely match the thermal loads imposed on the system, and are less capable of responding to changes not contemplated in original designs. Energy efficient buildings have unopenable windows, keeping contaminants generated within, inside the building. Most buildings recirculate up to 908 of conditioned air, and up to 508 of heating air. This situation causes a gradual buildup of pollutants because of lack of fresh air to dilute them.

Summary of causes of reduced air quality in buildings:(37)

* Sealed building envelopes

* Reduced ventilation rates, mechanical and natural.

* Dependence on mechanical HVAC systems

* Introduction of unstable synthetic materials, maintenance products, and finishes that off-gas toxic contaminants and respirable particles, or leave dust residues.

* Dependence on fluorescent and other gas vapor lighting that emit non-iodizing radiation

* Photocopiers and VDT's emit radiation and airborne contaminants and may require special environments for safe use.

* Methods of identifying and measuring concentrations of pollutants have improved.

To alleviate these problems, improved air distribution, humidity and temperature control is required, together with source, dilution and removal control of contaminants.

\subsubsection{Air Contaminants}

Air contaminants can be characterized as particulates (solids or liquids) and gases. Some are merely annoying, others are potentially harmful.(38)

Particulates are described in terms of mass concentrations, expressed as micrograms per cubic meter. These include all particle sizes or total suspended concentration. Large particles are more of an annoyance than a health hazard since they lodge in nasal passages. Respirable suspended particulates (ranging up to 3 micrometers in size) are important for health, because they can lodge in the lung. Among the major sources of contamination are: 
* Radon- a radioactive gas which decays into solid alpha emitters which can attach to dust particles and surfaces. Sealing of foundations, ventilation of basement areas and an increased outdoor air supply lower levels can alleviate this problem.

* Formaldehyde- from tobacco smoke, insulation, resins in paneling, furniture, cloth, carpets and adhesives.

* Carbon Monoxide- from tobacco smoke, furnaces

* Particles - from asbestos, fiberglass, fire retardents

* Radon Particles-from ground, cement, bricks

* Organic Chemicals- from copiers and paints

* Nitrogen Oxides- from heaters

* Micro-organisms- from people, plants; incubated in furnishings, ducts, cooling towers

* Allergens- from dust, insects

* Carbon Dioxide- from human breathing, combustion.(39)

6.7.3 Tight Building Syndrome

Tight building syndrome refers to epidemic complaints of illness or discomfort including headache, burning eyes, irritation of the respiratory system, drowsiness and fatigue. The symptoms are generally experienced for an extended time and occur in sealed buildings. The cause is difficult to determine, but often suspected to be related to building components or the air supply system.(40)

In 1979, a survey of 1100 office workers from 9 buildings in New York City was undertaken. The purpose was to investigate "tight building syndrome" complaints. Among the major complaints were:

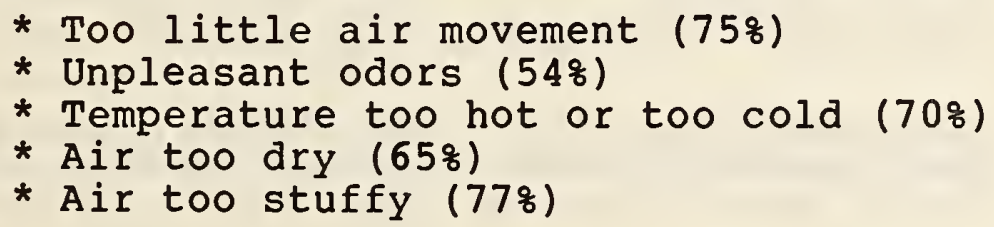

Among the health related symptoms noted were:

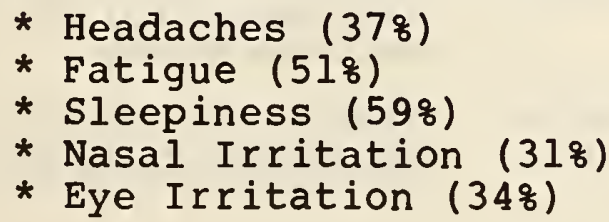

A consistent association was found for all relevant symptoms with the ability to control ventilation. A potential problem is also the production of photo-chemical smog. All the necessary conditions exist in offices to produce this smog- ultraviolet radiation from photocopiers and fluorescent lamps, formaldehyde (off-gassing from particle board, insulation), hydrocarbon vapors (off-gassing from paints, plastics), chemicals from solvents, cleaners, copiers.

Nine features common to building problems were identified:

1. A sealed building envelope. Insufficient fresh air drawn into building to conserve energy. 
2. HVAC systems disperse irritants throughout buildings. 3. Location of vents and exhausts can introduce outdoor contaminants into building. Poor placement of supply and exhaust vents can prevent exhaust from escaping, or reintroduce exhaust air into air supply.

4. Location of ventilation diffusers. Inlets and outlets are often in ceiling, sometimes short circuiting supply air, resulting in pockets of dead air and poor circulation. 5. Lack of individual control of environment contributes to stressful conditions.

6. Use of new furniture materials and cleaning agents.

7. Fluorescent lamps may provide energy for photochemical reactions among pollutants, creating indoor smog.

8. Parking garages, restaurants within office developments may add substantial amounts of combustion products to air. 9. Reduction of fresh air supply; lowered velocity of ventilation, which also reduces effectiveness of filters.

6.7.4 Control Measures for Indoor Air Pollutants; Responsibilities (41)

Ventllation: dilution of indoor alr with fresh outdoor alr or reclrculated filtered alr, using mechanical or natural methods to promote localized, zonal, or general ventilation

Source removal or substitution: removal of Indoor emission sources of substitution of less hazardous materials or products

Some modification: reduction of emission rates through changes in design or processes; contalnment of emisions by barriers or eealants

Air cleaning: purification of indoor a1r by $8 a s$ adsorbers, alr filters, and electrostatic precipitators

Behavioral adjustments: reduction in human exposure through modification of behavior patterns; faclittated by consumer education, product labeling, bullding design, warning devices, and legal llability
Radon and radon progeny; combustion by-products; tobacco smoke; b1ological agents (particles)

Organic substances; asbest1forw minerals; tobacco omoke

Radon and radon progeny; organ1c substances; abestif orw anerals; combustion by-products

Particulate matcer; combution by-products; blological agente (particles)

Organ1c substancea; combustion by-products; tobecco moke
Local exhaust of gas tove enlosione; alt-toalr heat exchangers; bullding veat1lat1on codes

Restrictions on sook1ng in public places; removal of esbertos

Platic barriere to reduce radon levelo; containsent of abestos; design of bulldiage Whout beseaente to avold redon; cetalytic oxidetion of $\mathrm{CO}$ to $\mathrm{CO}_{2}$ in keronese burbers

Reeldent1el alr cleanere to control tobecco - moke or wood soke; ultraviolet 1rradiation to decontaninate vent1lation alr; formidohyde orbant filter.

Swoke-free zones; arch1tectural deslgo of Interior epece; certification of formidehyde concentratson for boee product. 


\section{INDIVIDUALS}

* Maintain and properly use products and appliances.

* Exercise direct control of ventilation

* Restrict smoking in common areas

* Report incidents of degraded air quality

* Do not unnecessarily introduce contaminants in workplace

\section{BUILDING OWNERS OR MANAGERS}

* Operate and maintain a balanced ventilation system in compliance with codes.

* Use zone ventilation or local exhaust for indoor contaminant sources.

* Properly use cleaning solvents, paints, varnishes, herbicides, furnishings and insulation.

* Train staff in the limited use of potential contaminants, and their application in proper concentrations.

* Limit smoking where feasible.

ARCHITECTS, DEVELOPERS, CONTRACTORS

* Identify protection of indoor air quality as a design objective.

* Design ventilation systems to comply with ASHRAE standard 62-1981.

* Provide for separation of occupants from indoor pollutant sources.

* Specify materials and systems that minimize pollution risks, e.g. copiers.

* Provide containment systems for unavoidable pollutants.

* Provide exhaust fans for specialized "dirty" areas. 


\section{ELECTRICAL POWER SYSTEMS}

\subsection{FUNCTION}

Provide an electrical environment for the safe, reliable operation of office automation equipment and other electrical systems. This is the most critical system for office automation. The value of the information to be protected is a major factor in determining power protection needs. The other key elements are the dependability of the power source and the susceptibility of equipment to power changes.

The electrical environment for computers includes their power sources, grounding and electrical interfaces with communications lines, air conditioning, and life-safety systems. It also includes lighting and other electrically operated equipment. The electrical environment in which office equipment operates must also be considered, since electrical disturbances propagate through conductors, pipes, metal ducts and conductive structural members, or by radiation as radio waves. External sources of electrical fluctuations range from lighting to electrical loads generating electrical noise when operated or switched on and off. Internal sources of electrical changes can be caused by the office automation equipment itself. (42)

If absolute protection is needed, an on-line UPS is probably needed. This system regulates and conditions power fluctuations in addition to maintaining power during temporary outages. For organizations concerned with power outages, a standby UPS might be sufficient. This system costs from one-third to one-half of an on-line system. Standard switching times - the interval from the time that power fails until the UPS is initiated-are in the 2-8 millisecond range; within the 10-15 millisecond buffer storage of most microcomputers. (43)

Other power protection options include a variety of line conditioners and monitors. These provide varying degrees of protection in regulating electrical noise, surges, transients, and sags. Line monitors measure the current on electrical lines, permitting users to determine the effect of fluctuations on computer performance. Some monitors also keep track of temperature and humidity levels, which can cause operational errors and even failures.

\subsubsection{Background}

The quality of electrical power varies considerably from place to place. The power received at an unprotected workstation is likely to be susceptible to fluctuations which can result in problems ranging from lost data, to disabling a microcomputer. Consistency of power also depends upon location. For example, a company located in a multi-tenant office building is likely to be affected by other electrical usage in the building; e.g. motors cause fluctuations. 
Equipment characteristics determine susceptibility to power fluctuations. Mainframe computers are engineered to be tolerant of power changes, while microprocessors do not contain similar safeguards; they would add too much cost to the system. They are available as add-ons.

No equipment is immune to electrical disturbances but sensitivity to such changes varies from device to device and from one type of disturbance to another. Disturbances of high energy content can result in catastrophic failure of impairment of circuit components. Smaller disruptions may not damage components but may disturb logic signals and cause intermittent errors in data or control functions. Significant improvements in logic design and circuit integration ironically has made office systems more susceptible to disruption by electronic noise. These circuits need less power than their predecessors, and the quality of commercial power hasn't kept pace with these requirements. The faster response times of newer, high performance logic circuits makes them susceptible to fast noise pulses which have always existed, but failed to disturb the older, slower logic circuits. This implies that electrical environments suitable for older generation office machines may need improvement to accommodate newer systems. Power and electrical system requirements should be carefully reviewed prior to installing new systems. Separated clean power outlets are often needed for computer equipment.

\subsection{CRITERIA}

* Power sources should be relatively free of major fluctuations.

* The means to reduce or eliminate sources of electrical disturbances is needed.

* Circuits should reduce the coupling of electrical noise and transients from all sources into low power level circuits.

* The office equipment purchased should not be readily disturbed by power abnormalities.

* Software and operating procedures should readily detect, correct and recover from errors and power outages.

* Spare capacity is needed in electrical bus risers to handle future needs, e.g. to main and distribution panels. * Power panels are required for station-to-station distribution.

* Use power distribution modules for isolation.

* Segregate power usage ("clean" vs. "dirty" power), e.g. coffee maker should not be on same circuit as an office device.

* Consider using uninterruptable power systems for critical operations.

* Properly ground equipment for safety and operational purposes, e.g. lightning protection.

* Equipment rooms should be large enough to provide equipment with proper clearances and have proper HVAC. 


\subsection{POSSIBLE PROBLEM AREAS}

Problems originate in all parts of the power subsystem: (44)

* Primary Energy Source - public utility

* Converter to Electric Power - from steam power to rotary mechanical energy of the generator - to electrical power * Distribution System- wiring

* AC-DC Converter - convert from delivered power to DC, needed by computers

* DC Distribution System -

* AC Distribution System - needed to run motors, relays, lamps, etc. These components are tolerant of dirty power, but in the network there are many generators of noise and disruption and constantly varying loads, separated from the power company's delivered power. All can be disruptive to digital systems.

Power line pollution can be divided into three basic categories: overvoltage, undervoltage, and noise. Devices and systems are available to deal with each of these problems. The most severe problems in the electrical environment of an office are caused by overvoltage, which typically occurs in short bursts as surges or spikes. (45)

A power surge is an increase of voltage levels from 10 to 20 percent (or more) above normal, usually lasting from one half cycle to several seconds. A surge can be induced by static discharges, lightning, or inductive switching produced by starting or stopping a major electrical motor on another circuit, e.g. an elevator or air conditioner motor. A third, less frequent cause is power restoration after an outage. Surges generally do not affect the performance of traditional motor driven machines, but computers and microprocessors have critical threshold operating voltages, and power fluctuations can seriously impair their performance.

Spikes, which are sudden but brief disturbances reaching thousands of volts with frequencies reaching to the megaherz level, are also a danger to the operations of electronic office equipment.

Lightning does its damage directly and indirectly. A direct "hit" is possible, but very unlikely. However, when a bolt of lighting contacts a transmission line, or even comes close to the line (thereby inducing large voltages), the effect can be devastating to a computer.

In the case of man-made activities, spikes in the range of thousands of volts are commonly caused by a variety of circumstances; for example, when a large motor or transformer is switched off, or when utility companies reroute feed lines to adjust for changing consumer demand. 
Undervoltage occurs when the voltage drops below 120 , the nominal value used for most equipment. They can range from sags of a few volts, to complete outages. Most microprocessors are designed to handle voltage dips of 15 to 20\%; however greater amounts can result in the loss of data.

Electrical noise is probably the most universal a.c. line problem. It is broadly defined as high voltage, high frequency interference. There are two types-common mode, interference between a line and ground and transverse mode, interference across lines. The most common noise source is electromagnetic interference (EMI), often referred to as radio interference. Other common causes of interference are electrical motors and power lines that act as antennas for signals, and electronic ballasts in fluorescent lamp fixtures.

\subsection{POSSIBLE SOLUTIONS (36)}

\subsubsection{Suppressing Overvoltage Transients (46)}

Several methods are available to suppress overvoltage transients:

1. Dedicated power lines are used primarily for computer installations. The power is obtained from an inverter fed by an uninterrupted power source (UPS). This is an expensive method and therefore limited to critical instruments and controls that must be maintained on-line during power outages.

2. Isolation transformers installed between the ac power line and the equipment serviced are helpful for some voltage transients, but are not effective against high frequencies; secondary windings in the transformers can compound the problem. The addition of regulators, separating the primary and secondary windings of the transformer can overcome this difficulty.

3. Surge suppressors- The only way to guard against the effects of spikes and surges is to prevent them from from reaching sensitive equipment. This is accomplished by surge suppressors, which limit them to a size which protects data and devices. They employ sophisticated semiconductor devices to detect, limit, and dissipate spikes very rapidly. The best location for the surge suppression device is at the power input of the equipment being protected.

4. Voltage Regulators - supplies constant voltage to equipment; sometimes called constant voltage transformers or voltage conditioning equipment. Voltage regulators protect against "brownouts", and increased voltage levels. They control the power supplied to equipment, keeping the level within the required operating range. However, when there is a power failure, standby power supplies and UPS are needed to ensure that data are not lost. 
5. Power Distribution Equipment - Multiple plugs to distribute power to PC and peripherals. Can utilize features for short circuit prevention and circuit switching.

6. Monitors provide accurate diagnosis of power line quality by detecting and identifying all common a.c. line disturbances. Some can record ambient room temperatures also. In the simplest models, indicators light up and sound an audible alarm. More advanced models provide printouts of interruptions; summary reports of what went wrong, when, etc. They can be positioned upstream, nearer the power source, to document the status of incoming power; or downstream, from the power conditioner to record the power characteristics reaching computers or other devices. (47)

7. Power line filters are used to eliminate the undesired signals. They are designed to block out all frequencies except the desired $60 \mathrm{~Hz}$ of the power line. These filters do not eliminate surges and spikes.

8. Electromagnetic Interference (EMI) SuppressionElectromagnectic interference may be conducted along power lines or radiated through space. Power line EMI filters at the equipment input, and proper grounding of lamp ballasts and metal lamp fixtures will reduce both forms of EMI.

\subsubsection{Emergency Standby Power}

The UPS and standby power supply are designed to overcome the problems of power outages. Their goal is to provide required power to ensure against loss of data and interruption of normal operations of electronic equipment. Both devices get the energy needed for generating AC from the same source-batteries. Before the computer can make use of battery power, direct current of low voltage, it must be converted to $A C$ and then increased to standard line voltage using a transformer. The device that makes this conversion is an inverter, which converts DC battery power into $\mathrm{AC}$.

Standby power is normally off and does not start manually or automatically until the public utility power fails. The power is provided by a bank of batteries and/or diesel generators, which can be started within ten to thirty seconds, if their rapid starting condition has been maintained. However, this length of interruption would shut down electronic office equipment not served by an uninterruptable power system. Power surges should be controlled when this source is activated.

Standby and UPS are limited by the capacity of the inverter (maximum power available) and the battery capacity (duration of backup available). A few minutes of backup is usually sufficient to take actions to save the data by placing it in a permanent storage medium, e.g. a disk.(48) 
Speed is a critical factor in backup systems; they must act almost instantaneously if data are not to be lost. A standby power supply combines battery power and an inverter with a fast switch. The UPS uses a large battery charger with an inverter inside it. It is connected to the microprocessor and constantly draws power from batteries, thereby being isolated from the power line and the problems associated with "dirty" power.

The batteries supplying the inverter can only supply a limited amount of power, and standby backup consists of rechargeable batteries and a recharger, which keeps the batteries fully charged. The standby system is activated by a switch when the main power fails. The power outage must first be sensed, and then the switch activated. These operations, while fast, take some time, and can cause problems with ongoing activities. 
Table 15. Basic Cause, Effect and Treatment of Power Line Disturbances

\begin{tabular}{|c|c|c|c|c|}
\hline Condition & Probebility & Cousese & Effect & Trentiment \\
\hline $\begin{array}{l}\text { Line volteges too high } \\
\text { or low }\end{array}$ & $11-12 \%$ & $\begin{array}{l}\text { Nolghboring increases in } \\
\text { demand, loading down } \\
\text { eupply. }\end{array}$ & $\begin{array}{l}\text { Improper equipment } \\
\text { operation. Possible } \\
\text { demage if sustained. } \\
\text { Overheating. }\end{array}$ & $\begin{array}{l}\text { Constant Vottege } \\
\text { Regulator (CVR) }\end{array}$ \\
\hline $\begin{array}{l}\text { Sovere power drops or } \\
\text { outages of short dura- } \\
\text { tlon (20 milleseconds } \\
\text { [thousandihs] to a fow } \\
\text { seconds) }\end{array}$ & $51 \%$ & $\begin{array}{l}\text { Electrical storms, power } \\
\text { equipment foult. Addition } \\
\text { of heary cquipment to } \\
\text { load. }\end{array}$ & $\begin{array}{l}\text { Total discuption of } \\
\text { computer operations. } \\
\text { Demege to cquip. } \\
\text { mont when power is } \\
\text { restored abruptly. }\end{array}$ & 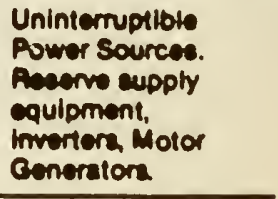 \\
\hline $\begin{array}{l}\text { Power surges and sags } \\
\text { of shon duratlons (typl. } \\
\text { cally a fow milliseconds } \\
\text { or a single power cycle) }\end{array}$ & $40-47 x$ & $\begin{array}{l}\text { Abruptly edding of remov. } \\
\text { ing loeds to the power llne } \\
\text { by ueere. Feulty or ereing } \\
\text { swltches. }\end{array}$ & $\begin{array}{l}\text { Fales computer out. } \\
\text { put, miereglstration } \\
\text { of logle clrcults } \\
\text { poselble hardwere } \\
\text { damege. }\end{array}$ & 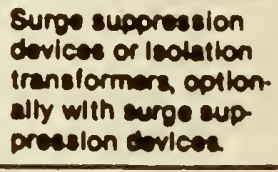 \\
\hline $\begin{array}{l}\text { High voltage impulses } \\
\text { of extremely short dure } \\
\text { tion (a fow microseconds } \\
\text { to a short manosecond) }\end{array}$ & $35-40 \%$ & $\begin{array}{l}\text { inductive load switching. } \\
\text { contect erce, startstop } \\
\text { equipment operation, } \\
\text { otalle discharge, electri. } \\
\text { cal storms. }\end{array}$ & $\begin{array}{l}\text { Premature compo- } \\
\text { ment faliure miereo- } \\
\text { istratlon of logic } \\
\text { circultry, equipment } \\
\text { down time. }\end{array}$ & $\begin{array}{l}\text { Surce suppreasion } \\
\text { dovices. }\end{array}$ \\
\hline High irequency RFI IEMI & NA & $\begin{array}{l}\text { Other computers, radio } \\
\text { and communication } \\
\text { devlces, amall motors } \\
\text { and commutators. }\end{array}$ & $\begin{array}{l}\text { Lille ellect on } \\
\text { digltal equipment. }\end{array}$ & Low pess filtera \\
\hline
\end{tabular}




\subsubsection{Uninterruptable Power Systems (UPS)}

The UPS is continuously connected to the electrical system through its inverter, always supplying power independent of the AC electrical line. Battery power is constantly maintained by a charger. All power is twice transformed; from AC to DC and back again to AC, a wasteful procedure in terms of energy. The advantage of such a system is that power dips and surges never reach equipment, because the UPS system is between commercial AC power and the equipment at all times.

UPS have become a virtual necessity for powering electronic systems where the application serves a critical need, e.g. a life safety system. These systems, unlike the emergency standby power systems, are always in operation (except for planned maintenance, or outages). UPS systems are typically supplied with sufficient battery capacity to carry a data processing load for periods ranging from five minutes or less, to several hours. The success of a UPS system depends on its ability to permit operations to continue without interruption; requiring power switching to be performed in less than one half cycle without substantial transient noise during the switching.

The most important feature of the UPS is its capacity, in voltamperes (VA), which must be sufficient to run all of the equipment that the UPS or backup power is connected to. Another key to selecting equipment is how long the system can run. The object of backup systems is not to function indefinitely, but provide sufficient time for an orderly shutdown when necessary, preserving all data, closing active files, etc. This can usually be accomplished within 5-10 minutes. Other important characteristics are the time required for recharging, and the waveform shape; sinusoidal is the best for most equipment. 
Table 16. UPS GUIDE TO INSTALLATION, MAINTENANCE (49)

\section{INSTALLATION}

* Have wiring, protection systems and disconnects been specified by the supplier?

* Has proper cabling been specified to prevent excessive voltage drop between UPS and critical load; UPS and battery supply?

* Is the building power adequate?

* Are the computers adequately grounded?

* Is the load protection coordinated with UPS protective devices?

* Is bypass power fed from a separate circuit breaker?

* Is air handling equipment adequate for UPS heat load and temperature limits?

* Who will install the equipment; should the supplier supervise?

* How will equipment be tested; is a formal procedure available?

MAINTENANCE

* Is there a monitoring system for UPS status?

* Is there a plan for responding to a UPS alarm?

* Is special test equipment needed; is it available?

* Are spare parts needed?

* Does the supplier offer preventative maintenance?

* Does the supplier offer training programs?

\subsection{Battery Features:(50)}

The older wet cell batteries are typically mounted in open-frame, two to three tier racks. They are shipped wet and charged for full-capacity startup. Conventional lead-calcium, wet batteries offer high reliability and require little maintenance and are easy to install. They do generate hydrogen when charged so ventilation is important.

Lead-antimony batteries or lead-acid stationary batteries are functionally equivalent to the lead-calcium type, but they have only half the life span, require more maintenance and monthly charging to maintain cell capacity.

Nickel cadmium batteries are the most expensive, are compact and light and have excellent temperature maintenance features; they perform well at both high and low temperature extremes. They also require monthly recharging and if maintained properly will last as long as lead-calcium batteries. 
* Use separate feeders and branch circuits for office automation systems so that no sudden common voltage drop will be created by non computer equipment sharing the same circuits ("clean" power).

* Large motors which drive air conditioning compressors, regularly started and stopped should not be powered from the same feeders or isolating transformers which supply the $O A$ power.

* The operation of $O A$ equipment from high impedance power sources such as diesel or gas turbine driven engines can result in large transient voltage variations; low internal impedance and/or larger kVA sizes can minimize this risk. * Avoid apparatus with regulators susceptible to overheating or to oscillatory interaction with loads containing their own regulators.

* The input power to equipment should be filtered to ensure proper performance. 

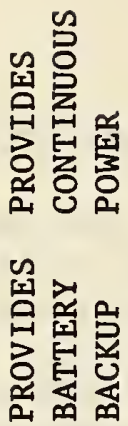

$\stackrel{\infty}{1}_{\infty}^{\infty}$
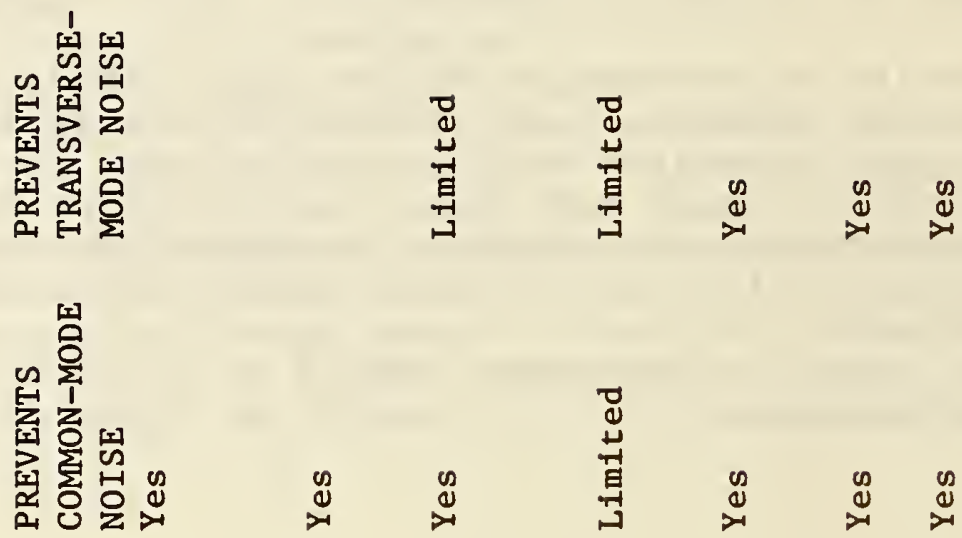

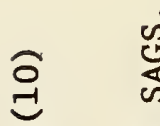

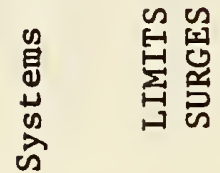

\& $\sum_{\infty}^{\infty} \sum_{-1}^{\infty}$

号



$\stackrel{\infty}{\infty}$

$\stackrel{\infty}{2}$

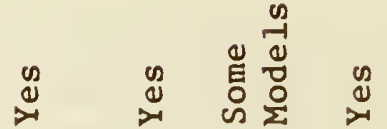

崩㖉

葛

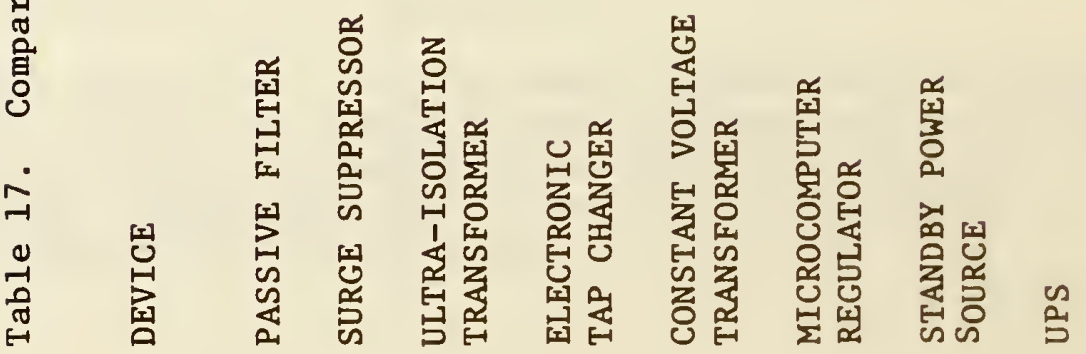

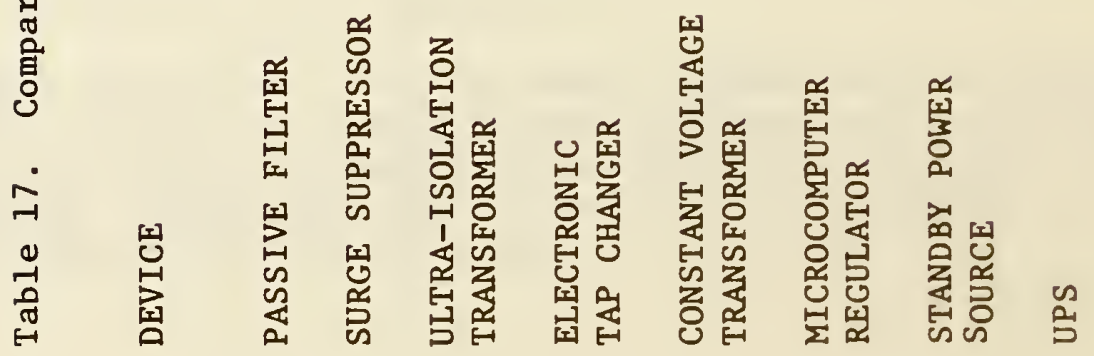

$\stackrel{\infty}{\infty} \stackrel{\infty}{\infty} \stackrel{\infty}{\infty}$

$\sum_{2}^{\infty}$ 


\subsection{ENVIRONMENTAL CONTEXT}

\subsubsection{Specialized Space Needs}

UPS systems and barriers should be in protected utility areas. Batteries represent potential danger from shock hazards, acid spills, flash heat and sparks from high-energy short circuits, or even explosions. They should be installed in dedicated battery rooms with controlled access, with temperature maintained between 70 and 80 degrees F, to ensure long life and capacity. The UPS hardware should be placed close to the batteries to avoid unnecessary loss in the dc bus and excessive electrical noise or possible shock hazards. Typically, the UPS is placed in a basement utility room or utility corridor area where the equipment can be properly ventilated and kept at the appropriate temperatures. The space should be secured and locked to prevent unauthorized entry. Appropriate warning signs should be strategically located. A shower is needed if batteries are of the lead-acid type. Finally, rooms should be shielded from potential sources of electromagnetic and/or radio interference. Telecommunications and PBX rooms might have to be secured because of the sensitivity or criticality of information. Keylock, security card or manned systems can all accomplish this purpose. 


\subsection{FUNCTION}

Wiring systems provide power and communications services wherever needed to facilitate organizational performance in offices. To provide flexibility to cope with changing requirements.

\section{2 BACKGROUND}

Wiring systems in offices have remained the same for years, but within the past decade this situation has profoundly changed. Manufacturers now offer new systems, designed to provide flexibility in delivering power and communications to work areas and running wires to, and in switching lighting fixtures. They also provide for greater capacities and security for communications wiring. The central role of individual workstations in automated offices has accelerated improvements ranging in complexity and sophistication from "intelligent buildings" to flat conductor cable. The impetus for these changes stem from:

1) the growth of the open-plan;

2) the advent of the electronic office;

3) energy conservation;

4) labor savings in running wiring and connecting it to lights and outlets.

Providing power and communication services anywhere requires a wire conductor within a conduit (sheath, raceway, plane under carpet squares, raised floors, etc.) connected to a terminal within the room and/or above the ceiling. Flexibility varies among systems - and initial costs vary accordingly. Under carpet cable can go anywhere. Wiring below access floors is possible anywhere there is an "electrified" panel. With cellular flooring and underfloor ducts, flexibility is governed by the spacing of electrical cells, but can be enhanced by the addition of under carpet systems.

The wiring approach taken depends on the likelihood and frequency of change in workstation location and/or configuration. For example the number and spacing of service fittings in an electrical grid configuration can dictate the degree of flexibility available (assuming no flat-conductor cable system). Every underfloor system shares the requirement to penetrate the actual floor surface, but the particular method used can be as simple as a lid, or as complex as a hole drilled through a concrete slab. 
In wiring several factors must be kept in mind:

* Flexibility. Examining needs for 5 to 10 years in the future. LANs, voice/data communications and PBXs all have special needs to be considered. Twisted pair technology is employed typically for PBX switches; if high speed data transmission is contemplated, proper insulating media is needed to accommodate this function. Many LANs today use twisted pair and coaxial cable; the latter serves as a main trunk. Other uses for coaxial cable are for broadband and baseband systems for interconnections to mainframe computers and high density communications networks.

* Transmission clarity. All wires differ in performance, depending on the materials used in construction and their capability in transmitting clear signals. Three problems encountered with wiring are capacitance, attenuation, and crosstalk.

* High capacitance is a particular problem; it refers to the wire's dielectric slowing of the signal resulting in loss of data or the distortion of information.

* Attenuation. This is a reduction in the amplitude of a transmitted signal and can result loss of data or the introduction of system errors.

* Crosstalk. This refers to the crossing of information from one circuit to another.(52)

* Media choices for present use and flexibility for future changes and/or installation are critical.

* The gauge of the wire and the permissible number of twists permissible per foot are important features.

* A wire survey of existing installed wire should be made, and updated when changes are made.

* Information concerning conduit sizing and space availability for current and future needs are required.

* Wire sheathing requirements for current and future needs should be specified. 
Great flexibility is needed for general purpose office buildings where tenant activities often exhibit high workstation density and medium-to-heavy communications needs with frequent tenant changes and rearrangement of offices.(53)

In planning for network growth, there are three primary factors to be considered:

* Expanded network utilization by the original users.

* Extension of the network to new users.

* Physical plant or campus modifications.

\subsubsection{SOME PROBLEM AREAS}

A major problem for the designer of the intelligent building is the wire distribution system; especially for open space planning which eliminates the walls that have traditionally housed wiring. The movement of advanced technologies into offices is evolutionary.

Installing and operating many different types of cable is to be avoided. It is not unusual for almost 508 of terminals and telephones in place to be moved within one year. Proper planning of wiring space must accommodate long and short term communications needs. The cost of wiring in existing buildings goes up with the height of the ceiling. A popular approach is prewiring; installing cables and raceways (cable conduit) during construction. This reduces installation costs and permits careful labeling and recording of cable runs and circuits, making changes easier.

A critical decision is selecting the types of cables and wires linking advanced data processing and telecommunications systems. The quality and dependability of these systems will determine the performance capabilities of other systems. Proper selection of wires and insulation materials can overcome most problems. For example, teflon fluorocarbon resin has superior dielectric properties and desirable thermal and chemical features. It can transmit information over long distances without distortion very quickly. It also has lower installation costs because it can be installed without conduit, since it has low smoke and flame spread characteristics. 


\subsection{CRITERIA}

\subsubsection{GENERAL}

* Capacity. Sufficient physical space to accommodate cabling, connectors, and advanced equipment such as LAN's. * Convenience. Flexibility and ease of initial workstation installation and future changes.

* Cosmetics. The appearance at distribution and delivery points, and special treatment required.

* Credibility. Code acceptance, single system responsibility, reputation of manufacturer.

* Cost. Complete distribution and delivery cost; first cost to building shell, move-in; and ongoing costs.(54)

\subsubsection{SPECIFIC}

* Optimized flexibility is needed to accommodate future changes; tradeoffs must be made between frequency of changes, initial and long-term costs.

* Wiring and communications systems should be integrated when feasible, e.g. information and energy management; proper shielding is also required.

* Systems should be aesthetically acceptable.

* Wiring changes should not disrupt ongoing activities.

* Dedicated circuits should be used to maintain critical operations such as security, safety, and highly critical information services.

* When laying cable, future needs for communications and office automation systems must be considered.

* Complete wiring diagrams and records are needed, updated to reflect all changes automatically by CAD system if possible.

* Cabling should be identified everywhere it enters or exits a room.

\subsection{TYPICAL APPROACHES}

The building architect traditionally addresses structural and architectural matters having direct impact on in-building communications networks. Horizontal signal distribution on the building floor is largely dictated by the floor structure.

The architect and developer want to minimize space for communication because it is not revenue producing. Minimal space is provided for telephone closets and communications equipment; tenant space is used instead. The basic office structure is expected to have a lifetime of 50 years, but technological and organizational changes occur about every 5-7 years. Network changes are costly in terms of dollars and disruptiveness.(51) 
Providing power and communications requires a wire or fiber conductor within a conduit (e.g. sheath, raceway, raised floor) connected to a terminal within a room and/or above the ceiling. The flexibility provided varies from system to system, as do the initial and long term costs. However, power and communications signal lines require sufficient separation to prevent interference, e.g. magnetic coupling.

Open office systems has led to the popularity of underfloor systems for electrical distribution and communication. The replacement of permanent walls with partitions and dividers and the movement of desks from exterior walls have decreased the use of walls for power distribution purposes.

The work support system exists under the floor in one of five different horizontal planes. It can run along the underside of the slab (as in a poke-through system), within the body of the slab (in a concrete underfloor system), just under the top surface of the slab (fill and finish), or above the structural slab and below the walking surface (raised or access floor). Underfloor systems require the penetration of the floor surface. The penetration can be as simple as raising a hatch lid, or require drilling through the concrete slab. The location and appearance of the service fitting are important design considerations, as is the appearance of the floor after relocation. Some designs treat service fittings as a necessary functional element to be clearly exposed and detailed, while others attempt to conceal them.

Equipment types, densities, and locations are frequently changed in a building's lifetime; power and communications systems must be designed to move and adapt. The options for distributing power, lighting, electronics and communications services consist of five basic approaches, often used in combination:

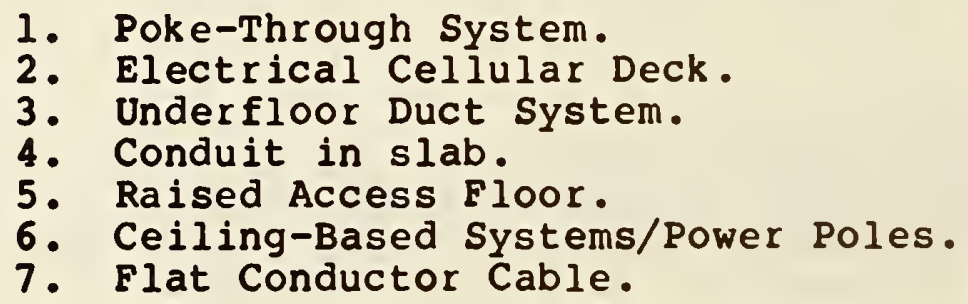


This system does not require any underfloor raceway. When a hole is drilled, a sleeve is forced into place and the service fittings is then installed from above. A fire-rated fitting is inserted into the hole, connecting either conduit or flexible plug-in wiring to it from the ceiling below. The conduit runs from its connection to the slab service fittings, to the currently spaced junction box. A sleeve may be preset during construction or after. The initial investment is low and outlets can be readily moved and the hole plugged. Too many holes can weaken the structure however.

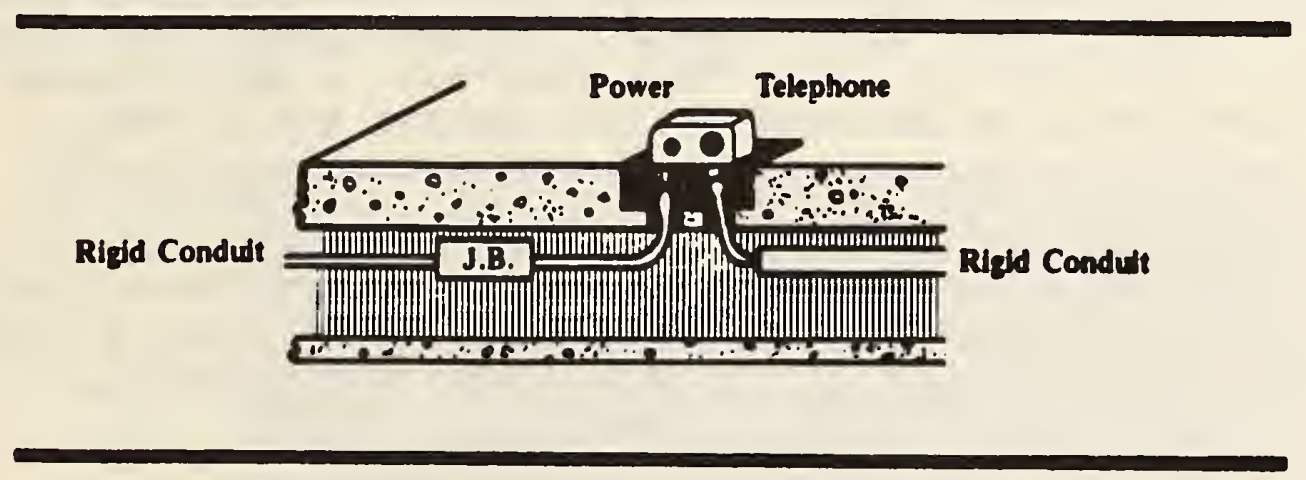

Figure 14. Poke-Through System 


\subsubsection{Electrical Cellular Deck}

The cellular deck is based on a corrugated steel deck containing electrified cells, with reinforced concrete on top. Cells run the entire length of the floor and provide services at predetermined locations through a short vertical outlet to flushmounted floor outlets. They share many of the features of the underfloor system; high first cost, flexibility and ultimate limitations for placement of outlets based on grid size - 6 or 8 ft intervals.

It consists of closely-spaced cellular raceways that connect at regular intervals to a main and header duct. A matrix of preset service fittings is spaced just below the surface floor in a trench. Service is obtained by punching a hole through a thin panel and bringing the appropriate cables through the opening. Physically, the wiring must penetrate the floor, and with carpet tiles, the carpet might have to be cut out or peeled back.

Cellular decks, like ducted systems, have trench headers that feed cables to parallel chases in the slab. The cellular deck contains the power, data and telephone cables within the enclosed cells of the floor deck itself. They also have a much greater capacity than ducts, which have outlets spaced about every 5 feet.

The system provides great flexibility with a minimal change in required floor depth to accommodate the system. Service fittings are sometimes flush with the floor, but frequently they project through the floor, causing an unsightly appearance and a potential tripping hazard. (See Figure 15.)

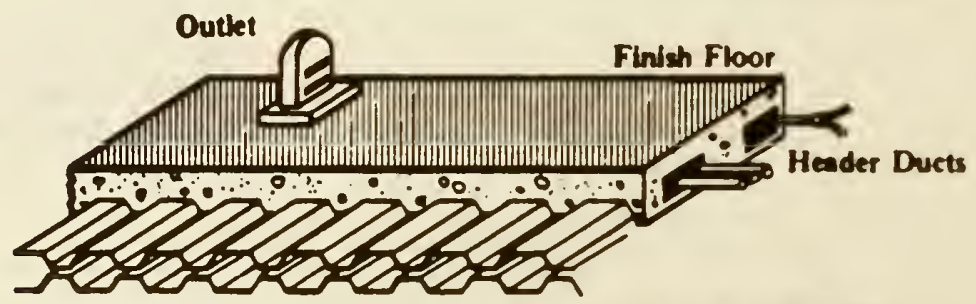

Receway for Electrical Wirlne

Figure 15. Cellular Floor System 


\subsubsection{Underfloor Duct System}

Underfloor ducts have been used in buildings since the 1930s; they usually contain raceways for power and space for telephone cabling. The system consists of metal ducts, between five to eight feet on centers, buried in the floor slab and connected by means of a vertical trench header duct, to electrical risers. The top of the trench header is flush with the finish floor and has electrical plates for wire access. The ducts are divided to separate power, data and communications channels. The ducts are non-structural, standing on top of the structural deck. Most ducts are now made of metal. The system has preset access points which allow minimal floor slab disruption, thus eliminating the need for fire safety precautions.

An underfloor raceway can run within the structural depth of the floor or be placed on the completed floor slab. Once the main trench duct has been placed, the branch ducts can be spaced at virtually any interval. Preset fittings poke up from the duct desired. Perhaps because of this inherent freedom, and the success of this approach that underfloor duct systems have been so little altered over the years.(See Figure 16.)

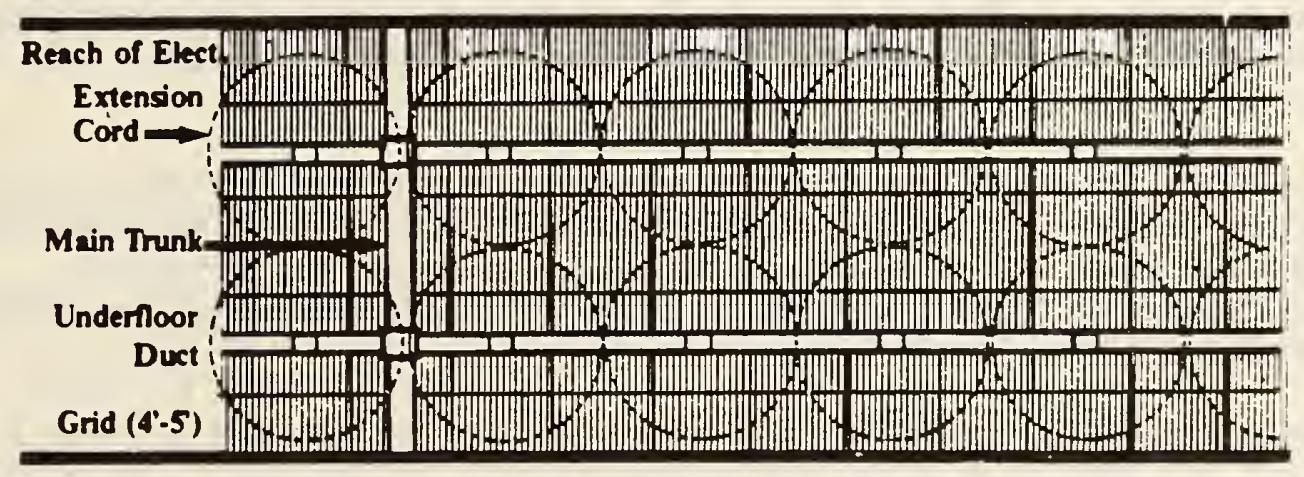

Figure 16. Underfloor Duct System

8.4.4 Conduit in slab.

Conduit is run from a power closet to a workstation. The conduit is suspended from hangars attached to concrete form, then is bent and brought up above the finished floor level. The floor is then poured and the wire pulled through the conduit. The system has little flexibility; outlet locations must be known in advance. The design usually consists of a crisscrossing of raceways installed in a reinforced concrete floor. Junction boxes are needed at every grid intersection. Access is through risers in the duct to access boxes in the floor. 


\subsubsection{Raised Access Floor}

Total access flooring is a system of interconnecting floor panels raised sufficiently above the structural slab to allow installation of electrical, mechanical, and air distribution systems. Access is possible anywhere on the floor by means of modular cabling systems. It is more expensive to install than other systems and requires considerable space. This approach is frequently used for the design of major computer facilities. Cabling may have to be plenum-rated if the underfloor area is a plenum.

This approach was originally taken to accommodate the special requirements of major computer facilities. Since many types of spaces require the use of conventional floors (bathrooms and washrooms), ramps, steps, or sunken floors have to be accommodated in some locations.

An access floor system has three major components: the panels, which form the working surface; the understructure supporting the floor panels from underneath; and the floor covering.

Selecting the proper support system is critically important. Two basic types exist. The rigid grid, snap-on, and stringerless systems are all kept in place by gravity. These systems are appropriate for computer floors but not for offices.

Bolted panels, secured to the pedestal and bolted to one another at the panel edge are appropriate in offices. They approximate the sound and feel of customary floors. A good structural connection between panel edges allows adjacent panels to share dynamic and rolling loads.

Three major types of panels are available. A woodcore panel consists of a one inch plywood or particle board core sandwiched between steel sheets. A potential problem is water absorption by the core, and it is not used in offices. The most commonly used panel is made of fabricated steel. This panel has a flat steel top sheet and a formed structural bottom sheet in a welded construction. A modification of this system features a steel panel with the interior cavity filled with a cementitious material; not concrete, because it contains no aggregate. This approach improves the acoustical characteristics of the floor.

The third type of panel consists of a concrete mixture; it is susceptible to impact loads and failure if overloaded.

The last part of the system is the floor covering. Carpeting can be laminated to the panel or tiles can be placed over the floor after installation. A good grade of commercial carpet will absorb the impact sound of foot traffic. 
Mockups of systems are an effective method of evaluating subjective features such as the "sound" and "feel" of the floor. These tests are best conducted with side by side comparisons of the systems being considered; it is difficult to make such comparative judgments in any other way.(55) (See Figure 17.)

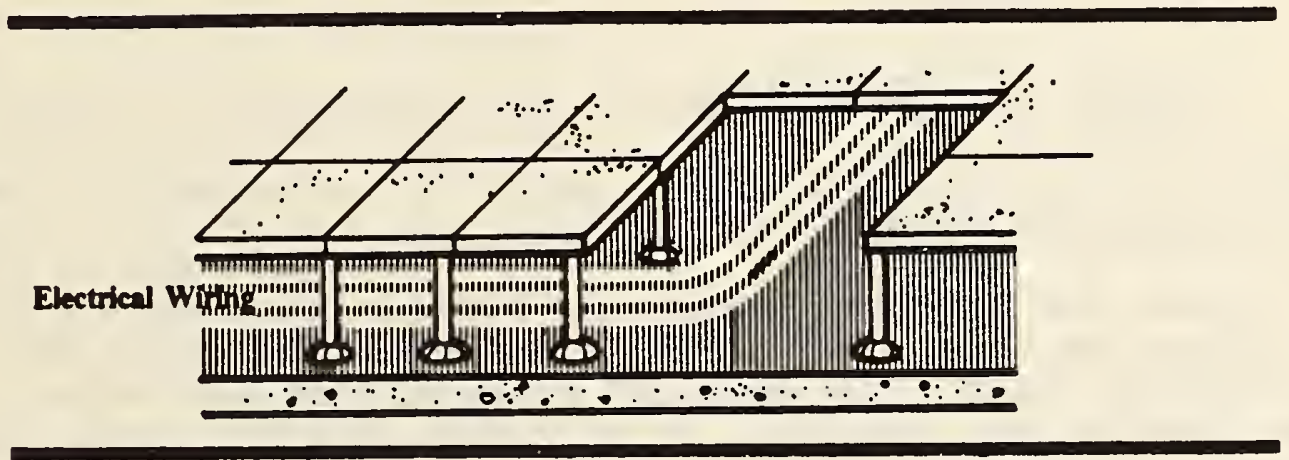

Figure 17. Raised Access Floor

\subsubsection{Ceiling-Based Systems/Power Poles}

These systems are an alternative to underfloor systems. Power poles are used as a direct means of distribution by vertical poles or flexible hoses located at workstations.

For the past 20 years, ceiling systems have been popular; they are inexpensive to install and easy to access. They are often used in conjunction with other systems to add more capacity; e.g. computer cables. The poles are metal columns which deliver wiring and cabling from a ceiling plenum to the floor, usually through enclosed ducts; outlets for power, data and communication are within the pole. Relocation of outlets requires unplugging and moving poles. They offer security to data lines since they run within the offices' ceiling plenums. They can be used together with partitions in open planning. A raceway at the bottom of a panel can bring lines to several workstations. The wiring capacity of raceways in panels and that of poles, is limited. (See Figure 18.) 


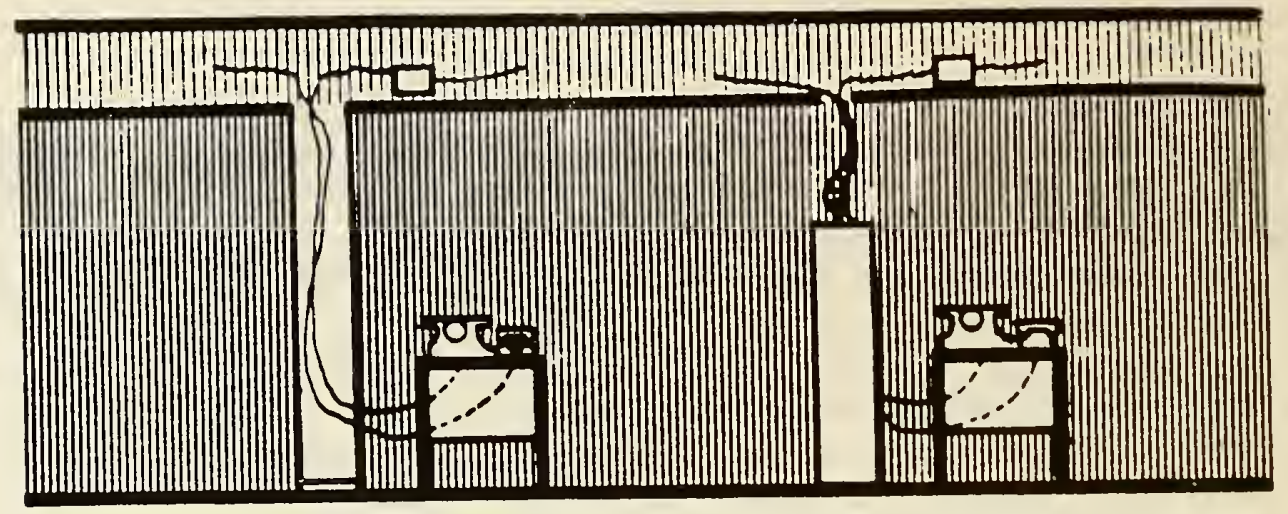

Figure 18. Power Pole

\subsubsection{Flat Conductor Cable}

This is a rather new approach receiving considerable attention today. It offers an option for wiring design in buildings where underfloor ducting cannot be used and conduit combined with exposed ceilings is not desired. Flat cable delivers power and communications services to floor pedestals (power outlets) that serve electric appliances and telephones. Good coaxial flat conductor cables are often difficult to find. (Pedestals should not be placed until workstations are assembled and last minute adjustments made.) Cables can be mounted directly on the floor (with a protective metal shield) under existing carpet tiles. Locational changes and/or new systems are installed by raising carpet tiles in the desired path, and redirecting existing flat cable runs or by tapping into a run. Connections between flat and round cables are made at transition boxes, usually located in walls or columns. A major consideration in the use of flat cable systems is to ensure that they are shielded against puncture or other contact. (56).

The following are benefits of these systems:

(1) Short installation time.

(2) Simplified specification of branch circuit wiring (e.g., no drilling).

(3) Maintained structural integrity of the floor system.

(4) Elimination of slab height increase needed with floor ducts.

\subsubsection{Flat Conductor Cable Checklist}

* Expect to see cable-caused ridges in the carpet tile initially; the problem is often a temporary one.

* The cable cover should not be reused; it will be distorted when pulled up, causing an uneven carpet tile.

* Consider keeping excess material for replacement purposes.

* Plan beyond the pedestal itself. 
* Determine the kinds of wire management needed between the pedestal and the using appliance.

* Determine the number of outlets needed for each workstation.

* Close coordination is required among the following systems: electrical, communications, floor (carpet tiles), and workstations.

* Use undercarpet telephone cable where appropriate.

* Breaks in the wire are difficult to locate.

* Turning radius may be a problem.

\subsubsection{Workstation Distribution}

This consists of pre-wired interconnecting panels, which eliminate the need for individual wiring. Many furniture systems have power in raceways, but power must be clustered at some point from floor, wall or column; planning of clusters for future expansion is important. 
8.5 TRADEOFFS AMONG ALTERNATIVE WIRING SYSTEMS

SYSTEM

POKE THROUGH

ELECTRICAL CELLULAR DECK

\section{POSITIVE FEATURES}

* Low first cost No raceway

* Delayed installation until rental

* Cables readily accessed

* Minimal visual intrusion
UNDERFLOOR DUCT
* Relatively flexible

* Changes are easy

* Minimal visual impact

* Integration of cells reduces costs, labor

* Large cable capacity and great flexibility

* Preset outlets easily relocated

* Simplified wiring organization

* Accessible

* Fire safe

* Minimum visual impact

* Preset outlets easily relocated

* Can be used with flat wire

* Simplified wire organization

* Cables protected from damage; secure
NEGATIVE FEATURES

* Expensive to change

* Disrupts activities

* Fire safety concerns

* Outlet relocation disrupts floor below

* Possible data security problem

* Limits on outlet spacing; total outlets

* Need to maintain fire and structural integrity

* Expensive

* Service fittings may protrude; hazards

* Added fire protection needed under activated cells

* Lengthy cable runs if flat cable not used

* Expensive

* Planning flexibility is difficult

* Less capacity than cellular deck

* Sheetmetal work required

* Nonstructural ducts need added slab in concrete buildings

* Wide duct spacing

* Long cable runs if flat wire not used 
TRADEOFFS AMONG ALTERNATIVE WIRING SYSTEMS (Continued)

$\begin{array}{ll}\text { SYSTEM } & \text { POSITIVE FEATURES } \\ \text { RAISED ACCESS } & \text { * } \text { System integration } \\ \text { FLOOR } & \text { facilitated } \\ & \text { * Flexible } \\ & \text { Almost unlimited } \\ & \text { cable capacity and } \\ & \text { routing } \\ & \text { Outlets easily } \\ & \text { relocated } \\ & \text { HVAC systems } \\ & \text { accommodated }\end{array}$

NEGATIVE FEATURES

* Installation expensive

* Changes sometimes

dis ruptive

* Some spaces need conventional floors

* May require teflon treatment-air plenum

* High initial cost

* Some systems can't accommodate heavy loads

* Some systems have hollow sound when walked on

CEILING-BASED
SYSTEMS

FLAT CONDUCTOR CABLE
* Easy to integrate with furniture systems

* Flexible

* Short installation time

* Floor integrity kept

* Used under carpets

* Readily moved

* No limits to spacing or routine cables

* Outlets quickly relocated

* Installation can be delayed to rental
* High first cost

* Planning critical

* Aesthetics

* Cable must be protected

* Careful installation needed to avoid ridges

* Expensive

* Turning radius needed

* Carpet tiles required

* Floor slab must be smooth

* Over lapping cables can create carpet lumps

* Damage from heavy loads

* Cable length limited to 50 feet

* Not allowed in some places 


\subsection{ADDITIONAL DESIGN CONSIDERATIONS}

\subsubsection{Retrofits}

Providing adequate methods for concealing and running cables from mainframe computers to peripheral equipment is often difficult in existing facilities. Standard solutions include running cables up inside walls and over ceilings, flat conductor cable systems, power poles, or raised flooring systems.

\subsubsection{Upgrading of Cabling Systems}

Substituting new technologies for old ones depends on the ability to remove obsolete systems because of limited capacity of horizontal and riser space. Cable runs should be visible and accessible over their entire run, and must meet fire safety requi rements.

\subsection{ENVIRONMENTAL CONTEXT}

* Hung ceilings with greater than average depth below the structural member, for coaxial cable, fiber optics, and future air conditioning ductwork. This can sometimes result in aesthetic problems because of incorrectly repositioned ceiling tiles or tiles chipped from repeated access for maintenance and/or changes.

* Floor to ceiling utility chases can be placed on a regular module. It is readily accessible, but reduces flexibility and limits room dimension and total space arrangement options available.

* Movable walls, floors and/or ceilings are available, with vertical and horizontal chases to accommodate future wiring needs.

* Plug-in cables can be used to connect above ceiling runways to power poles; these systems have been used traditionally for installing lighting fixtures. For enclosed offices, the plug-in cables can be nested in floor/ceiling assemblies.

* Underfloor soft wiring systems with plug-in boxes and harnesses of various lengths can be used to connect with pre-slotted outlets in raised floors.

\subsection{CHECKLIST WIRING SYSTEM DESIGN}

* With cellular flooring and underfloor ducts, system flexibility is governed by the spacing of electrical cells, but can be augmented by undercarpet systems.

* Maintenance areas must be designed with sufficient space for distribution boxes and panels for changes and planned expansion.

* Expansion space is needed in the building core to install additional bus risers for communications and power. 
* Extra sleeves are required for future power and communication cables.

* Additional riser space might be needed on each floor for office automation systems and networking, including mainframe and minicomputer linkages. * Ceiling heights of rooms must accommodate raised floors and/or ceiling plenums must be deep enough to accommodate present and future needs.

* Sufficient capacity is needed for feed conduit, vertical risers, telephone closets, and conduit to individual tenants.

* Specific needs of building users should be considered, such as industry specified requirements for medical groups, stockbrokers.

* Plenum space requires plenum rated cable approved by local fire regulation agency.

* Electrical needs for telephone/wiring closets. 


\section{COMMUNICATION SYSTEMS}

\subsection{FUNCTION}

Communications systems provide the means to transmit voice, image, and data information within an office or campus, and to outside services or mainframe computers.

\subsection{BACKGROUND}

Several types of communications and/or computer applications occur in offices. Soon, all workstations will likely have a terminal and at least one telephone. The computer may be serviced by dedicated hard-wired connections to desktop terminals, or by the same wires serving the telephone, possibly in a local area network. Other possible workstation services are electronic and/or voice mail, electronic filing, and facsimile transmission. The telecommunication system can have a major impact on the services for office workers. It will also have important implications for the design of buildings and systems, e.g. cable distribution, wiring closets, riser systems, main telephone room and service entrance. There is also a need to plan for future expansion of these services. The percent probable expansion in five year increments should be estimated. A useful strategy in planning such systems is to identify the known requirements and those which are not yet specified, and provide for the flexibility and/or adaptability to make changes in the future.

\subsection{CRITERIA}

\subsubsection{GENERAL}

* Ubiquity. Access must be comparable to a telephone system, and terminal hardware should be inexpensive.

* Simplicity. Sending a message should be as simple as typing a letter or using a telephone.

* Flexibility. The system should be capable of transmitting texts of any length in any format, and be able to send graphic information.

* Reliability. It must be available for use virtually all of the time.

* Privacy and Authentication. Messages should be protected from inspection by unauthorized people. Positive identification of the sender to the recipient should be available. Most systems employ passwords for this purpose. 


\subsubsection{SPECIFIC}

* Voice and data information should be readily transmitted at an acceptable rate of speed.

* Error rates of systems should conform to organizational requirements.

* Systems should be sufficiently flexible to facilitate future changes and/or expansion.

* System features, e.g. capacity, flexibility, bandwidth, should respond to office activities.

* Sufficient power is needed to meet emergency conditions, e.g. UPS.

* Systems should handle analog and digital information.

* Adequate protection is needed from electromagnetic and radio frequency interference.

* Systems should provide the means to integrate building support (e.g. energy management) and operational data. * Systems should be designed to facilitate maintenance. * A single organization should be responsible for system operation, and problem reporting; a "help" desk is useful. * A diagnostic capability is needed to locate problem - what and where. This should be located in a network control center.

* Voice and data communications channels should be separated, unless digitized.

* Video signals may be digital or analog; part of a local area network or separate. It may be installed with video conferencing, video text, CATV, security, etc. 97

* Systems should be designed to facilitate security in information handling and transfer.

* Voice and data closets should be provided; more room is required if security is of primary importance.

\subsection{TYPICAL APPROACHES}

The telephone lines or cables cross the property line at the telephone service entrance, where they enter the building. These lines interconnect with the building cable system at the main telephone terminal equipment room, the "heart" of the building's communication system. (In multi-storied buildings, more than one main terminal room may be needed.) From the main terminal room(s), riser systems distribute the lines to each building floor. The particular riser system design used depends on the type and layout of the building and the systems installed. There are two basic types of systems - closed shaft and open shaft. The closed system consists of vertically aligned closets on each floor, connected by pipe sleeves, slots, or conduit through floors. In the open shaft design, the riser cables pass from floor to floor, without being enclosed in closets. Early coordination with the local telephone company is essential during initial planning. 
9.4.1 Checklist of Network Features (57)

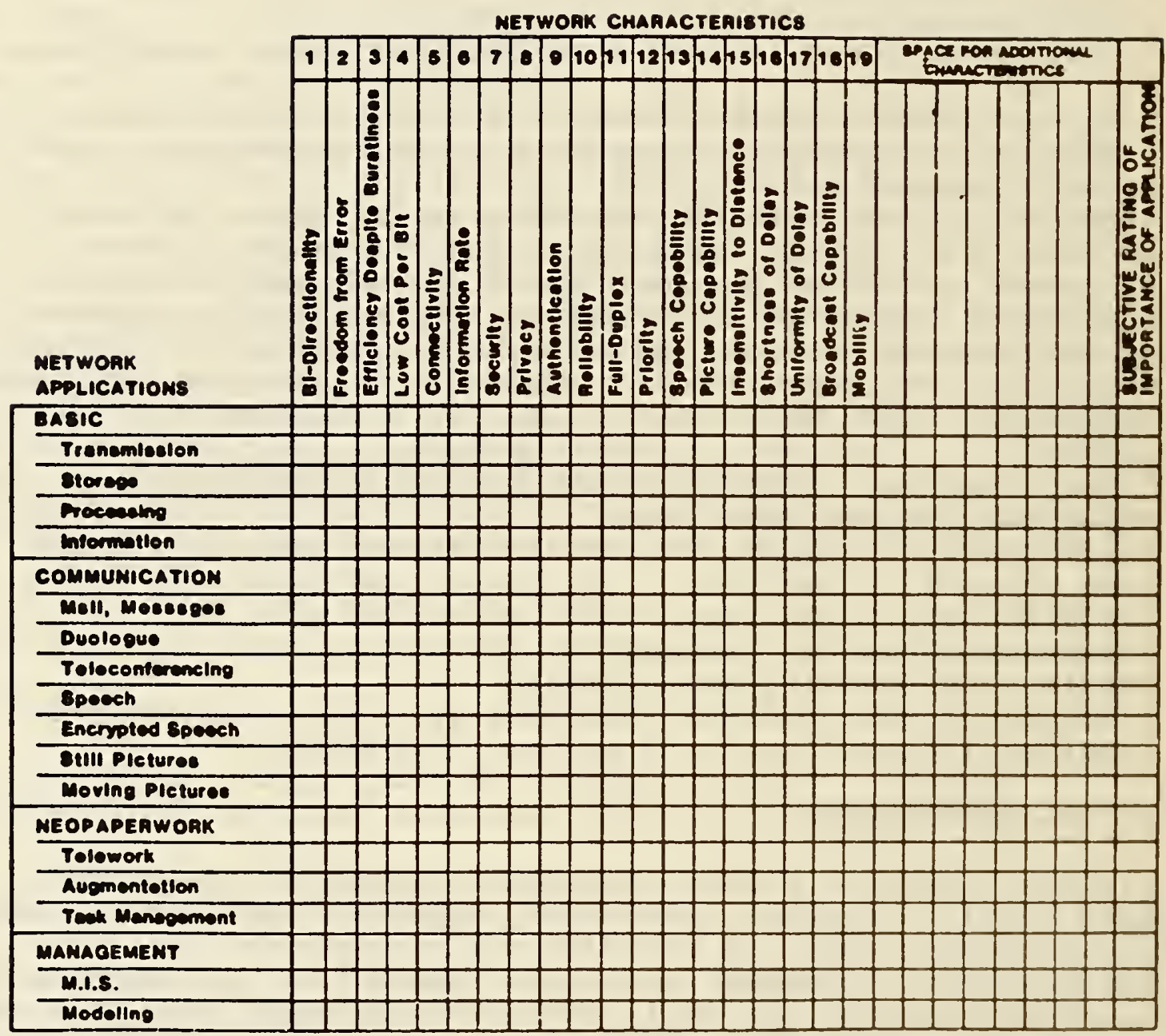

Figure 19. Network Applications and Features 
The framework for defining a communications network is based on a highly layered series of protocols developed by the International Standards Organization (ISO), called the Open System Interconnection (OSI), consisting of seven communication layers: (58)

1. Physical- defines the electrical and mechanical network interfaces. It specifies the signaling means, e.g. baseband, the modulation technique used, station identification addresses, etc.

2. Data Link - where the actual packet formats are established, with particular access control mechanisms used to regulate physical network use. Data are encapsulated in packets containing physical addressing information, error detection, etc.

3. Network- determines how messages are transmitted from one network to others, since many paths may exist. It may use several intermediate steps to move information to its ultimate destination.

4. Transport-provides another level of connections between network entities. It manages the connections and segments messages into pieces that the network can support. It may also be involved in error and flow control.

5. Session- used to set up and break communications paths across the network and manage the exchange of data. It is responsible for multiplexing and demultiplexing messages, managing the sequencing and priority of messages and providing needed buffers.

6. Presentation- responsible for making data available to the Application layer in a meaningful fashion. It takes care of protocol conversion, data unpacking, translation, or encryption.

7. Application- provides for the identification of users and services; responsible for initiation and reliability of data transfers, general network access, flow control and recovery. Utility programs may perform network file transfers, terminal to network support, etc. 
9.4.2 Checklist - Design for Communications Systems

* Telephone Service Entrance

* Size is determined by the number of lines needed, present and future. (Except for fiber optic systems)

* Main Telephone Terminal Room

* Location should be close to the center of the riser cable distribution network.

* It should be convenient to the service entrance.

* Environmental conditions should be appropriate for the equipment supported.

* The size should house existing equipment, accommodate necessary activities and planned expansion.

* If a non-sealed battery powered or battery backup for nonsealed PBX is installed, separate space should be available for the batteries.

* Cabling

* It must be housed in conduits where required, and riser ducts separated from power conductors.

* It should be protected from sources of electromagnetic interference.

* Networking

* Internal systems should be linked with external ones, e.g. public, private, LAN's, telephone, microwave, satellite.

* Security impacts 
Wiring is run into central distribution points, and individual connections made there. They are used to distribute voice and data to and from terminals and devices. They can also facilitate wiring circuits, house power supplies and control devices

The floor area served by any given closet is limited by the space available for cables to enter and leave the closet. This is usually about $10,000 \mathrm{sq} f t$, but may reach upward of $50,000 \mathrm{sq} f t$. Having multiple closets on each floor helps reduce cable-drop lengths, facilitates the addition of more drops, and makes rearrangement of cables easier.

Cabling between wiring closets, workstations, and other devices is achieved by several means. Underfloor raceways should be accessible during initial construction and later expansion. An underfloor is usually assembled and installed using feeder and distribution ducts, junction boxes and other modular components. This design can put constraints on the structural design needed to accommodate the ducts. The cellular floor is an integral part of the building support structure, with distribution cells made of steel or concrete. A trench header is a specially manufactured metallic trough (maximum depth of 2.5 inches) with a cover that fits flush with the finished floor; it is normally used as a feeder duct for cellular or underfloor configurations.

\subsubsection{Advantages of wiring closet:}

* It allows communication network installation, alteration, and maintenance with minimum disruption of ongoing work. * Noise generated by communications equipment or workers can be contained and controlled.

* The internal environment of the wiring closet area can be readily managed and controlled.

* Network security can be ensured by locking closets.

* During initial building construction, wiring closets can be located in areas to provide optimal cable distribution.

\subsubsection{Wiring Closet Checklist:}

* The wiring closet should be located at the point offering the best access to the cabling distribution. * Access is needed without disrupting normal office operations.

* Doorways should be at least 36 inches wide, 80 inches high, and without doorsills.

* All doors should open outward and be equipped with locks. Data communications closets usually have standard 19 inch racks with universal hole spacings. * Walk-in and shallow (if they open into circulation areas) types are both used. They should be at least three feet deep unless they open into a circulation area.

* Floor space should be open, without center posts. 
* Within the closet, carpeting or uncovered concrete should be avoided. Carpets create static electricity while concrete is a source of dust.

* Walls of three quarter inch plywood for mounting telephone punch-down blocks and strain-relief cabling should be installed.

* The racks are usually bolted to the floor, but can be wall mounted. A minimum sized closet provides one rack for each 100 drops, and space for one additional rack for future expansion.

* Closets for data systems should contain patch panels to cross connect data devices.

* Easy access to risers is needed.

* Sufficient power should be provided for equipment and systems.

* Closets should be protected from sources of electromagnetic interference.

* Environmental conditions should support equipment.

* Lighting should be from incandescent sources. The light source should be overhead and distribute a shadowless light.

\subsection{TELECOMMUNICATIONS ACCESSORIES (59)}

\subsubsection{Functions}

There are self-contained, plug-compatible devices for word processors, small computers, terminals, and other types of office automation equipment. Once connected they provide direct and automatic access to one or more domestic and international networks.

\subsubsection{Criteria}

Desirable features are as follows:
* Capability to support multiple private and public networks.
* Access to a text editor.
* Call dialing, connect, disconnect, and reentry features.
* Capability to address commonly called locations.
* Communications port options allowing for selection of one, two, or all business networks.
* Compatibility with dissimilar types of equipment through standard accessory ports.

\subsubsection{Checklist of System Requirement Needs}

* Appropriate protocols, speed conversions and signaling must be perfectly matched with those of the networks accessed

* A careful maintenance capability

* An integral battery back-up system to protect the memory from momentary power outages 
* User-friendly programming features, with instructions in jargon free language

* Multiple user capability, connecting dissimilar systems. * Low power requirements, lightweight, and low heat output

* Modular, expandable and capable of upgrading

* Capability to perform simultaneous operations - e.g. receiving and sending messages, information status, etc. * Good record of customer service, preferably through local dealers and users

\subsection{ELECTRICAL/COMMUNICATIONS LINKAGES}

Four approaches are available - twisted pair, coaxial cablebaseband and broadband, fiber optics. Considerations for their selection depend upon a number of factors:(51)

* The capacity required

* The distances between proposed stations

* The importance of data speed

* The requirements to interfere with other systems

* Acceptable error rates

* Proposed expansion requirements

* Maintenance requirements and constraints

* Cost considerations - first and long-term

* Availability

* Existing conditions

* Manufacturer or supplier

\subsubsection{Twisted Pair}

This is the oldest technology and consists of two or more wires connecting devices to one another, sometimes employing PBX for central switching. Limited bandwidth makes it inappropriate for high speed data and television. Lack of shielding makes it susceptible to interference from sources such as AC power lines and mechanical equipment. They need large, expensive ducts to carry the many required wires; trouble shooting and fault isolation is difficult because of the number of wires.

It consists of paired copper conductors in a sheath; used for vertical and horizontal signal distribution within a building. It is used with PBX equipment to switch to the terminal equipment. Twisted pairs can produce crosstalk between wires and can pick up unwanted signals from external sources such as telephone cables in an electrical closet. (It is a dispersive medium.) Shields are provided to minimize such problems (between pairs and for groupings of pairs.) The greater the effectiveness of the shield however, the greater the mutual capacitance of the cable, resulting in poorer data transmission characteristics. Also, the more pairs within a common shield, the greater the difficulty to bend and place cable and the higher the cost. Large shielded cables are both heavy and stiff. 
The major benefit of twisted-pair technology is its reasonable performance at a low cost per foot at maximum data rates of $1-4$ kilobits per second. With proper design rings and bus topologies are accommodated.

\subsubsection{Baseband}

These systems are especially useful within a building or a campus. They avoid the need for dedicated lines; communication is transmitted on separate channels. They offer plug-in installation, are relatively trouble free and easy to repair when difficulties arise. Taps must be made at specified locations along the cable. Connecting baseband with other systems requires interfaces.

The system is placed on one channel of a coaxial cable or a TwX cable and users must take turns sending and receiving. They send one signal at a time at 1-10 megabits per second. The signals can be sent via time division multiplex, thereby enabling several users to use the system simultaneously. It is a nondispersive medium, the signal being confined within the shield placed around the central conductor. Baseband doesn't require the dedicated wires associated with twisted pairs.

Baseband systems can connect computers, word processors and other office automation devices on a single floor or limited area. They can support high data rates but are not generic; many different types are available with widely different electrical

characteristics. They cost about $\$ 4$ a square foot, which minimize their usefulness in serving individual workstations. Wiring dimensions are large, presenting problems for cable runs in small plenum spaces favored in new buildings.

Among other shortcomings are susceptibility to electromagnetic and RF interference; also cabling cannot extend more than a few thousand feet without expensive digital repeaters. Unlike twisted pair technology, baseband systems do not permit ready cross connections. Taps are used to connect devices to networks sometimes and are prone to noise interference.

\subsubsection{Broadband}

Broadband systems work best over large geographic areas (many kilometers)! using inexpensive amplifiers. Information is carried up to distances of more than 40 miles by several hundred channels on a cable television type of coaxial cable. The channel bandwidth can accommodate voice, video and data channels at high transmission speeds.

This is an excellent vehicle for a generic telecommunications utility. Many signals can be sent concurrently; each on a different frequency at $1-5$ megabits per second. Transmissions require radio frequency (RF) modems to convert digital to analog signals, and then back to its original digital form. It allows 
each workstation universal access to a common signal

transportation utility. A user can communicate anywhere there is physical connection to the cable; cross connections with twisted pairs is not needed. Connections between workstations are changed by allocating different frequencies; analogous to the switching function performed by PBXs through common time slots.

Broadband systems have high maintenance costs since they require a staff of trained $R F$ technicians for design, daily maintenance and careful tuning of components to proper frequencies. Both the cable and the broadband hardware are extremely sensitive to temperature and humidity changes.

\subsubsection{Fiber Optics}

Fiber optic systems use light beams traveling through glass threads. Each fiber can carry many channels, and many channels can be packed into a relatively slender cable. The bandwidth is considerable, as is the capacity to accommodate network system terminals.

This technology employs light signals, pulsed in fine glass strands (about 0.005 inches in diameter) to carry a stream of data at extremely high rates. Light can be modulated in shorter duration and lower power pulses than electrical signals (light has shorter wave lengths). Receivers can also detect signals containing less energy than is required for telephone modems.

The principal advantages are its sturdiness and its inherent security. Fiber optic systems are immune to physical and electrical influences and apparently are almost impossible to tap. They are difficult to use for local networks for the same reason; precise alignment of thousands of fibers is needed to ensure a continuous connection.

Table 18 presents the cable characteristics of various media. 


\subsubsection{Tradeoffs Among Cable Types} POSITIVE FEATURES

\section{TWISTED PAIR}

* System compatibility through the telephone system

* Availability of wiring

* Inexpensive

BASEBAND

* High speed data transmission

* Relatively trouble free
$(60)$

NEGATIVE FEATURES

* Limited capacity

* Slow transmission speed

* Lack of shielding:

susceptible to interference * Large ducts needed for wire

* Problem detection difficult

* Connecting to other networks requires interface devices

* Requires ducting, conduit or fireproofing to meet codes

\section{BROADBAND}

* High speed data transmission

* Doesn't require conduits or

* Relatively expensive protective ductwork, when special cable used

* Can be fastened directly to walls or ceilings

\section{FIBER OPTICS}

* High transmission speed

* Very high bandwidth

* Secure transmissions

\footnotetext{
* Limited to point-to-point

* Maintenance difficult

* New technology; operational

data limited
} needed

* Cable pulling required

* Interface needed to link to other networks

* Requires ducting, conduit or fireproofing to meet codes * Taps must be made at specified locations 
Table 18. CABLE CHARACTERISTICS

FEATURE

TWISTED

PAIR

1

$\star \star *$

INSTALLATION

$\operatorname{cosT}$

POTENTIAL FOR

INTERF ERENCE

SKILL NEEDED

TO INSTALL

MAINTENANCE

DIFF ICULTY

SUPPORTED

CONF IGURATIONS

KEYS

$\star \star \star$ HIGH

* * MODERATE

* LOW

*

$\star \star \star$

*

$R, S, B, T$
SHIELDED COAXIAL FIBER

TWISTED

PAIR

OPTICS

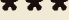

**

* *

$\star \star *$

* *

*

*

*

*

$\star \star \star$

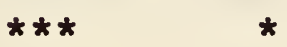

*

*

$B, R, T$

$B, R, T$

$S, R, T$

$$
\begin{aligned}
& \mathrm{R}=\mathrm{RING} \\
& \mathrm{T}=\mathrm{TREE} \\
& \mathrm{B}=\mathrm{BUS} \\
& \mathrm{S}=\text { STAR }
\end{aligned}
$$

Table 19 presents LAN meaia comparisons of varıous wiring approaches.

Table 20 compares features of LANs and PBXs from a functional standpoint. 


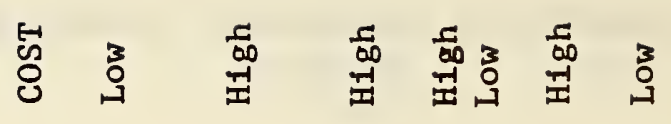

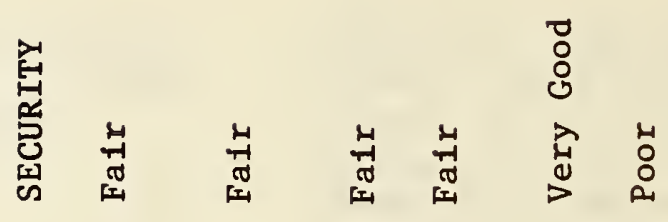

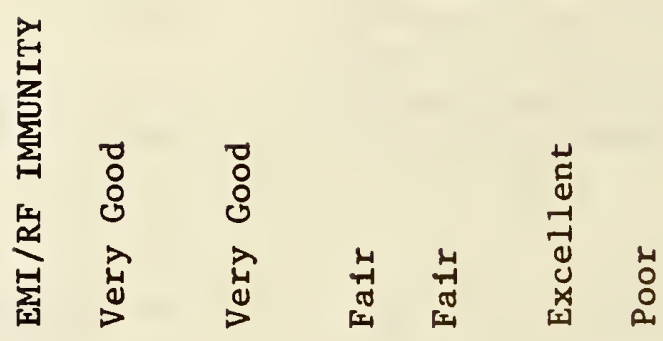

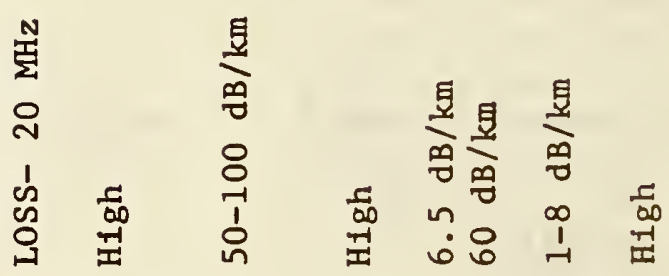

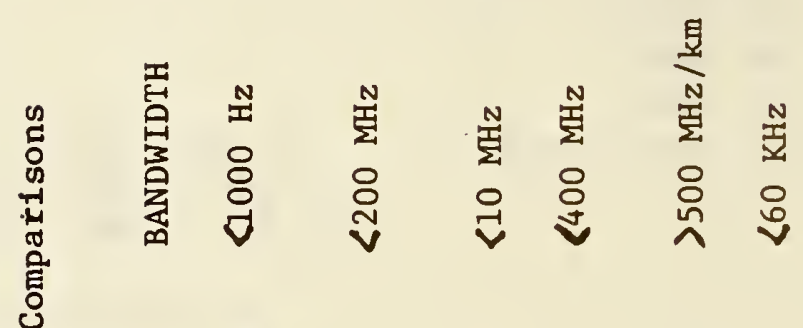

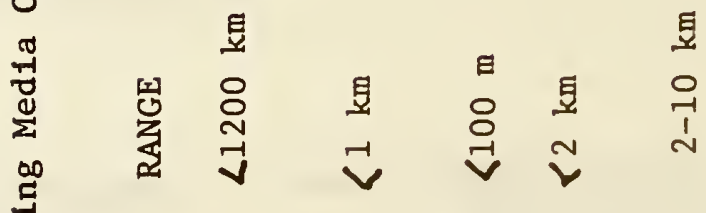

垩

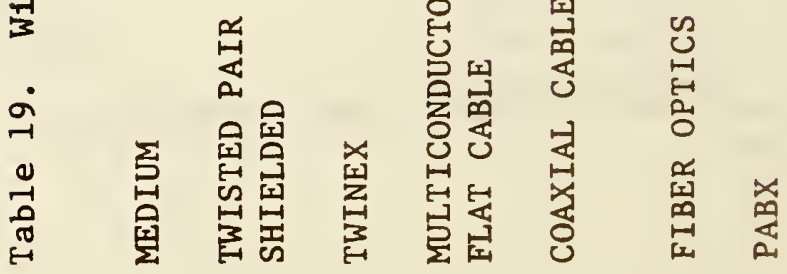


Questions that a manager should ask when choosing between a LAN a PBX are:

* How many users are expected to be on the network?

* Will the users need switched access to multiple hosts?

* Can you cost-justify additional cable needs?

* Are people expected to move frequently within space?

* Does the PBX have the capacity for long data transfers in addition to normal telephone traffic?

* Do you need remote access to distant data bases?

For PC and terminal users to send limited amounts of data at speeds below 2400 baud, the PBX is sufficient.

The wiring for both PBX's and LAN's is from workstations to wiring closets. In the PBX, the wire is then physically concentrated into trunks before being brought to the PBX. In LAN's, the network interface units are in the closets, and then connected into the major network cable.

\section{Table 20. Comparison of LAN and PBX features}

LANs advantages:

* when large amounts of data are transferred at high speeds

* when data protocol conversions are needed

* local work groups exchange data; less expensive

PBXs advantages:

* many users communicate with shared resource; high speed not critical

* combined voice and data possible

* existing wire can be used

* changes made readily without new wire needed

* permits ready billing for telephone usage 


\subsection{FUNCTION}

LANs provide the means for office technologies to communicate with one another. They are characterized by single organization ownership, within a building or among a small set of buildings, distances not more than a few miles, and switching capability which permits messages to be transmitted and received.

Establishing a LAN requires an understanding of how to best serve organizational needs. Typically such needs might include monitoring and control of building systems, data processing, security alarms, and the generation, transmission and reproduction of written documents. The selection of a particular system depends on how many of these elements are to be integrated into one coherent communication system.(62)

\subsection{BACKGROUND}

One way to think about LANs is a complete computer system, connecting small computers and peripherals together. A processor is dedicated to each user and a network is used for sharing data, or to access peripheral equipment.

A LAN is an electronic communication linkage which may not require public communications facilities to operate. It is designed to establish a "highway" to optimize network traffic and trouble shooting capabilities for linking different machines, languages, and operating techniques into a coherent system. A network can provide of advanced technology capabilities to every worker at every location. It also enables coordination of diverse activities, consistent with overall organizational objectives, e.g. management information systems, security and safety requirements, energy management, and centralized data bases $(62)$.

LANs must have enough communications capacity to transfer documents without extensive delay or the need to coordinate such actions with network users. Flexibility is very important; the more system design is tailored for specific types of equipment and/or physical locations, the less useful it is.

LANs should be easy to install and maintain and be user-friendly for laymen. Its should be as easy to use as a telephone. For example, users should not have to learn many screen formats or instructions. LANs should support the maximum data transfer rate between any pair of communications devices and any communication between devices operating at different speeds.

There is no single LAN networking approach that can meet all telecommunications requirements. A network should allow equipment from different vendors to communicate at varying speeds using 
different protocols. It should provide easy access without considerable rewiring.

The simplest LAN is one which links components from a single vendor. The highest level of sophistication are those systems which provide:

* Local processing capability of a personal computer network

* Intercomputer communications of a universal network

* Powerful data management capabilities

* High speed and sophisticated software, essential for handling voice and document image as well as data communication (63)

Networks can be divided into three general categories:(64)

1. Vendor specific; developed by computer manufacturers to support the end-to-end needs of their equipment. Their main function is to provide single vendor host-to-host and terminalto-host connectivity.

2. All purpose LANs have little affiliation to host vendors. They are broadband and baseband systems to provide connectivity and protocol support to a variety of vendor equipment.

3. New PBX's allow RS-232 and coaxial connection of workstations, support moderate to high data rates, and provide protocol conversion and device emulation. They offer the advantage of a single wiring approach to data and voice communication; many users can use existing twisted pairs.

In choosing a LAN, two issues are of prime importance: Will the network fit into the existing environment, e.g. is the wiring appropriate? Does it meet the functional requirements of the organization?

Among other important LAN factors to be considered are:

* Connectivity. It should provide physical connectivity equivalent to the vendor's standard approach; transparent to the user, devices should appear to be attached to standard coaxial, twin-axial, or twisted pair connections. It should be protocol transparent.

* Network speed. Flexibility in speed should be provided to accommodate device growth and increases in transaction volume. A common mistake is to demand too much speed and pay for unneeded capacity. Terminals and other devices must be able to utilize the speeds provided.

* Capacity. How much added capacity should be built in during initial installation? Provision for modular expansion at reasonable cost is essential. 
* Cabling. The cabling architecture should place as few restrictions as possible on cable type and length. Cables should provide easy access throughout site, and flexible enough to accommodate a variety of interfaces.

* Gateways. LANs must be able to communicate with external hosts, networks, users and other devices.

* Multivendor communication. The system should permit access to databases and equipment from many vendors. Protocol translation, device emulation and code conversion capabilities are needed.

The following tables provide information concerning the economics and market shares of LAN's.

Table 21. Cost Proportions of Typical LAN's

DESCRIPTION

DETAILED PLANNING

DETAILED INSTALLATION

DESIGN

HARDWARE COMPONENTS

INSTALLATION
PERCENTAGE OF TOTAL

28

8

34

32

Table 22. Market Share by Type (A.D. Little)-Percentage (65)

TYPE

BASEBAND BUS

BROADBAND BUS

TOKEN RING

CENTRALIZED LAN

THIRD GENERATION PBX

PBX WITH INTEGRATED LAN 10
1987

1992

20

15

15

10

10

10 (WITH PBX)

20

25

15

25 (WITH LAN) 


\subsubsection{PROBLEM AREAS}

The products of one vendor for accomplishing a function, e.g. electronic mail, are incompatible with those of other vendors. Furthermore, the software that runs one computer, commanding a printer or a disk, is often unique to a vendor, and cannot be used for a different system, even when sharing a cable. To help solve this problem, the Institute of Electrical and Electronic Engineers (IEEE) created project 802 to establish standards for hardware and software components of networks.

Careful system installation is needed for good performance. Places where the cable is cut should be marked and sufficient room provided where cable runs negotiate tight spaces and turns. If not mounted securely the cable can be twisted, which can lead to a cable break. A 360 degree cable loop is required where connected with a component, to permit reconnection if a break occurs. A weather tight shrink tubing seal should be used to protect the junction to the equipment housing.

\subsection{CRITERIA}

Two types of criteria are important for LAN, those associated with system performance, and those which address design issues.

\subsubsection{System Performance}

Table 23. Requirements for a LAN (62)

* Relatively high data transmission rates (typically 1 to 10 or higher megabits per second).

* Geographic distance spanning at 1 kilometer or more

* Should support at least several hundred independent devices.

* Simplicity, or providing the simplest possible mechanisms for the required functionality and performance.

* Good error characteristics, good reliability, and minimal dependence upon any centralized components or control.

* Efficient use of shared resources.

* Stability under high load.

* Fair access to the system by all devices.

* Easy installation of a small system, with gradual growth as the system evolves.

* Ability to optimize and report status of system elements.

* Ease of re-configuration and maintenance.

* High reliability of devices on network.

* Low cost.

* Easy maintainability.

* Maintenance service contract at low cost.

Tables 23 and 24 indicate technical requirements and throughput applications of LANs, respectively. 


\subsubsection{Design Issues}

* LANs should operate under typical environmental conditions of buildings in which they are located, with normal exposure to dust, humidity and temperature change.

* Equipment may be required to operate within given electrical constraints, e.g. power, voltage, phase, and/or frequency characteristics.

* Space is needed to accommodate specialized equipment and cabling requirements.

* Flexibility to accommodate changes and additions with minimal disruption to ongoing activities.

\subsection{TECHNOLOGICAL ISSUES}

First generation systems were designed to serve asynchronous terminals, not synchronous systems or PCs. They were designed for baseband transmission. Some present broadband systems support comprehensive network-measurement, performance and control facilities. These facilities simplify the installation and support of the broadband-based networking systems.

The Ethernet system, the first commercial one, employed CSMA/CD applications; the most widely used method today. This system requires that before transmitting data, each network interface listens to the media to determine whether any messages are being sent; if so, transmission is not made. The later systems employ token passing, where any station that has the token can transmit a message. (66)

The newer methods, coaxial cable and CATV technology are both designed to operate in harsh environments and share cost benefits from high-volume manufacturing processes. The price difference between baseband and broadband wiring is narrowing.

More than one local network is often needed to support the company wide mix of equipment and uses, requiring a linking of networks. Two devices are used to accomplish this, a bridge or a gateway •

A bridge is a simple device to link networks using the same protocols. It reads information packets transmitted on a network, and routes them accordingly to each network addressee. It doesn't modify the content or format. It provides a simple and efficient way to interconnect devices remote from one another. It is also used locally to guard against failure of an entire network when a problem occurs; it partitions an large system into smaller self-contained units. A bridge can also be used to maintain security; preventing distribution of sensitive data. 
Gateways are used to enable LAN's with different features to communicate with one another. Among the differences are:

* Addressing schemes

* Maximum packet size

* Protocols

* Time delays for data transmission

* Error recovery schemes

* Status and performance information

* Routing schemes

* User access controls

One type of gateway is a protocol converter, which entails developing connection schemes for each network pair. It accepts a packet from one network, strips off the control information and retransmits the data using protocols for the receiving network. At the other extreme is the use of standardized protocols usable by all networks. Unfortunately this standardization has not yet occurred. (67)

Table 24. Typical Throughput Applications of a LAN

APPLICATION DESCRIPTION

TELEX

COMPUTER TERMINAL

PHONE-LINE

MAXIMUM DATA RATE-

PRIVATE LINE WITH

CONDITIONING

DATA PHONE DIGITAL SERVICE

SATELLITE DIGITAL VOICE

TELEPHONE SPEECH

COMPRESSED DIGITAL VIDEO

BROADCAST COLOR VIDEO
APPROXIMATE THROUGHPUT (bps)

55

$2400-4800$

9600

$9600-56000$

$24000-32000$

64000

1.5-3 MILLION

92 MILLION 


\subsection{ORGANIZATIONAL ISSUES}

Network management can be complicated by LANs. Most management tools do not work on local networks. The evaluation of LANs is made on a cost per node basis. This must account for cable, interface devices, active and passive network components, interface boards, and software. Future costs should be estimated to determine the effects of expansion on the initial estimate.

Support for most networks requires that operators be trained in the use of configuration software. The complexity of the software varies from system to system as does the mode of change. Some changes must be performed at individual workstations; in other instances, they can be made centrally.

A company has three installation options: (68)

* It can install the network itself

* It can use third-party vendors and hire contractors and consultants for certain aspects of installation

* It can have the network designed and installed by a vendor that offers turnkey service.

The first option is the least popular one because it requires a high degree of expertise and involvement by the user

organization. It is likely that the installation will take longer and is more apt to result in problems than the other two approaches. Normal activities will have to be modified while personnel learn how to design and implement the system. However, they will be well trained to use the final system as a result of on-the-job experience.

The second option works best when at least one staff member is knowledgeable enough to develop design and performance specifications as well as acceptance criteria. A major problem is that a variety of vendors will be involved in putting the system together and coordination is likely to be a major task. Furthermore, overall system responsibility rests with the user, and when difficulties arise, it is difficult to determine which particular vendor should correct the problem. This lack of system responsibility by hardware, design and systems contractors is a major shortcoming of this approach.

The turnkey system is one which is now receiving considerable attention by users. The turnkey vendor develops the needed planning information, develops a workable design, installs the network and trains the staff in its use and then is responsible for maintaining the system. The one vendor approach simplifies equipment procurement and processing time, and therefore is the most timely method. A problem area is that the vendor might not be as responsive as in-house personnel when system malfunctions occur; response times to solve problems should be addressed in contract negotiations for such systems. 
Obtain technical documentation on features such as specific frequencies. used for each service, frequencies available for expansion and maximum the maximum distance terminal equipment can be located from trunk terminal. Also important are trunkinterface-device specifications, including number of ports, speed processor chips employed, and number of interface devices planned. The precise protocol for data transmission, the actual throughput expected and the services supported are also vital to know.

Services requiring special care should be noted, and the services critical to organizational needs to be optimized during the design process. The physical location of trunk taps should be identified, to ensure that planned expansion is facilitated. Planning should include using organizations, e.g. data processing and communications, and be coordinated with long range plans. The actual layout (topology) of wiring and its mounting, should be carefully planned and documented; vertical and horizontal distribution systems. The characteristics of the cable medium must be carefully considered during these plans, e.g. attenuation loss, amplification, accessibility for servicing, etc.

The growth limits of the networks should be clearly understood from the outset. If a branch is near its capacity, early redesign is preferable to the needs for major repair after installation.

The cable should be tested throughout its bandwidth, before installation. Finally, the installed cable must conform to building codes. (69)

\subsection{SOFTWARE ISSUES}

PC networks link computers operating under MS-DOS, a single user operating system. Performing network functions such as coordinating simultaneous updating of data files requires additional capabilities that must be added to MS-DOS. Every implementation requires a unique approach.

Network software consists of a set of programs designed to control the input and output of all the hardware in the system. These programs are needed at each network station and at the central server to take care of network functions. The first task of any LAN operating system is to allow multiple systems to share a large central hard disk, a server.

The server can be divided in many ways. The simplest one, allocates a portion of the disk to each user, who are the only ones permitted to access it. This system is not a true network since it does not permit sharing of data.

A network needs a system to assign and monitor read and write access privileges. Typically, this consists of files designated 
as private and public or shared. Private files are accessible by the owner only; public ones have read access to everyone, but not write access; shared files have read and write access.

With shared files, controls are needed to ensure file integrity. For example, when two users simultaneously are updating files, the last one to make modifications will overwrite the data added by the first user; thereby losing these data.(70)

\subsection{TYPICAL APPROACHES}

\subsubsection{Network Configurations}

Most local networks are designed to accommodate several hundred stations (nodes), separated from one another by various distances, sometimes as much as a mile. Each node operates as a device (terminal, computer port, printer, etc.) and a communication system, enabling data from diverse organizational elements tobe used by an individual working on a given problem or office function. Three types of configurations exemplify the structure of most networks.

\subsubsection{The Star}

This is the oldest arrangement, with a multiplexing device serving at the head of the network. Devices connect directly to the hub by coaxial cable, or a voice/data telephone, or by using some type of modem. The net is centrally controlled - resulting in a breakdown of the entire system when the hub encounters problems. (See Figure 20.)

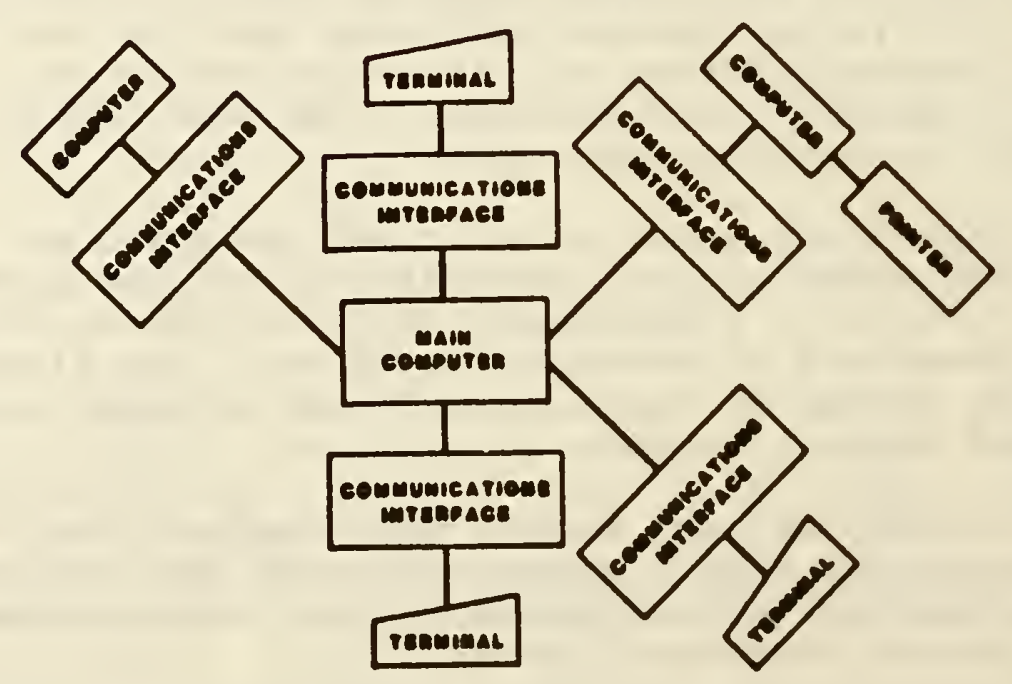

Figure 20. Star network topology 


\subsubsection{The Ring}

This configuration eliminates the need for a central hub switcher. A signal repeater is located at each node of the network, enabling signals to travel great distances. The ring permits the use of fiber-optic cable for transmissions, permitting very high performance. A major problem is that if one node goes down, the entire network is out of operation. Also, when a station is added, the network is down. (See Figure 21.)

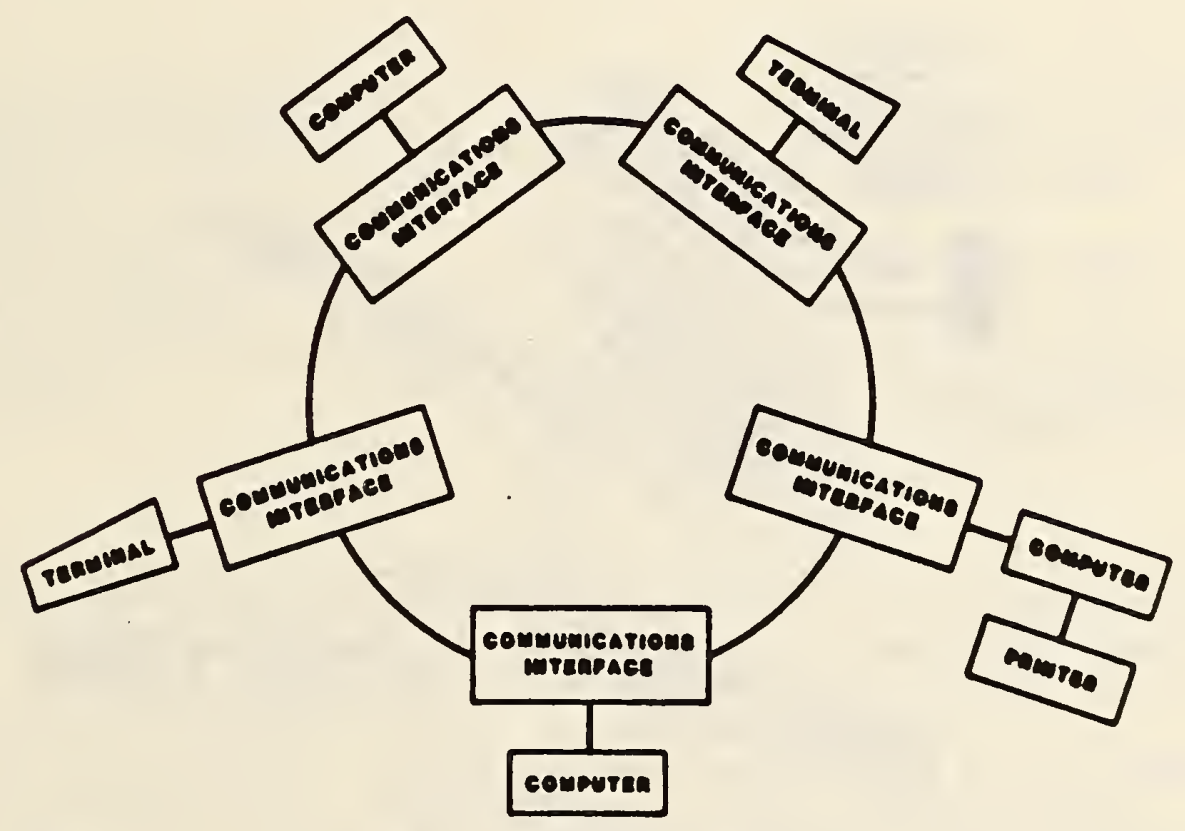

Figure 21. Ring network topology 


\subsubsection{A Bus Network}

This is the most common form of network, consisting of a length of coaxial cable (bus), to which microcomputer stations are attached by means of simple cable taps. There is no centralized control hub and signals from one station move along the bus in both directions to all stations tapped into the cable. This system is suited to a business environment because installation is relatively easy and cheap, without requiring system shutdown when changes are made. Also, it is reliable because there are no active components along the transmission cable. It does not accommodate fiber-optic cables. (See Figure 22.)

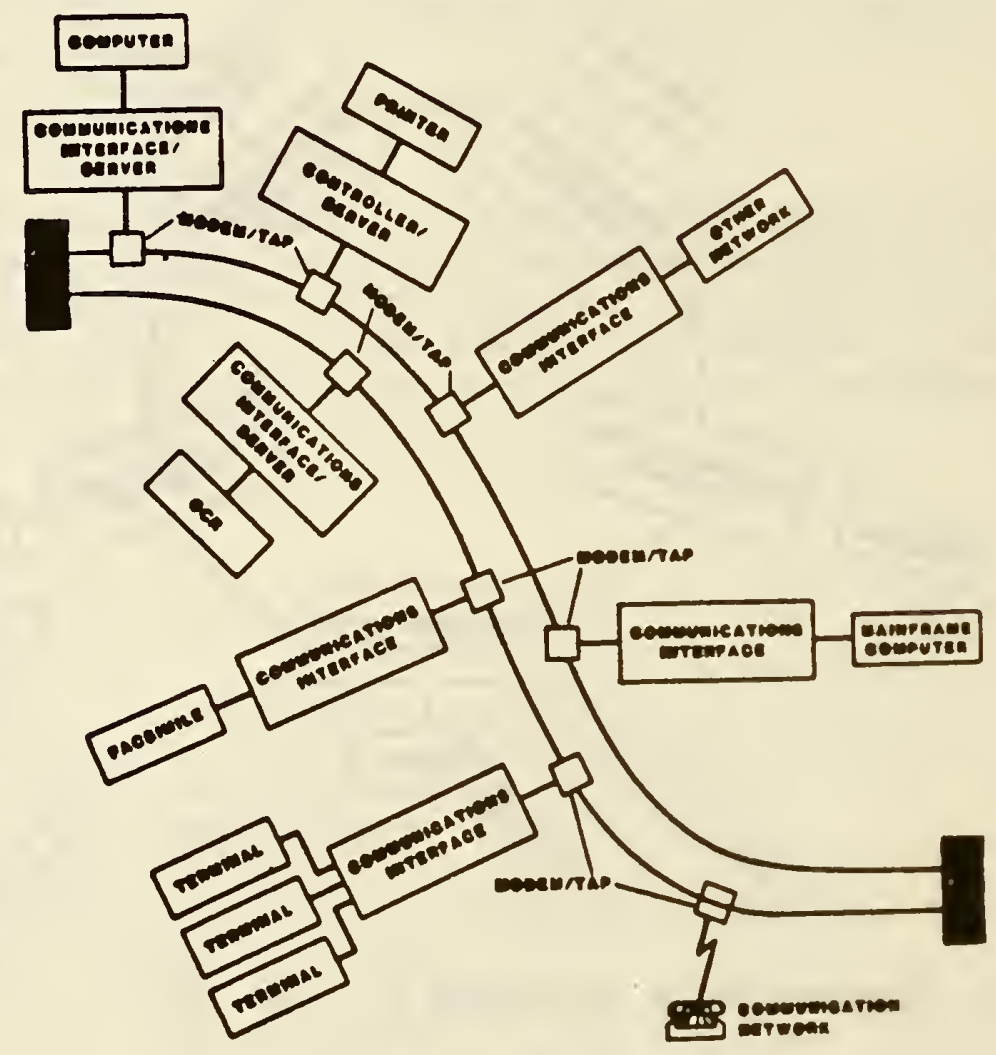

Figure 22. Bus network topology 
10.7.1.4 Tradeoffs Among Alternative Configurations of LAN

Table 25 indicates some important positive and negative features of each approach.

Table 25. Positive and Negative Features of LAN Configurations

POSITIVE FEATURES

STAR

* Multiple access possible

* Memory may reside in central and peripheral locations

* Ideal for monitoring system and individual performance

* Accommodates fiber optics

\section{RING}

* High overall performance

* All terminals can communicate with one another

* Supports distributed systems

* Repeaters used for greater

distance possibilities

* Fault detection and diagnosis from any terminal

BUS

* All terminals communicate with one another

* Control may be decentralized

* Widely used; good results

* Few maintenance problems

* Easy to add peripherals
NEGATIVE FEATURES

\author{
* CPU malfunction affects \\ entire system \\ * CPU limitations influence \\ system capability \\ * High load operations \\ require careful access plans
}

* Easy to access; security

problem

* Bandwidth limits data

transmission

* On-line access control

strategy needed

* System doesn't operate if

one node goes down; redundancy

and $\log$ ic unit needed. 


\subsection{ENVIRONMENTAL CONTEXT}

10.8.1 Space Considerations.

Among the considerations are:

* Room for the interconnection of units colocated with support units.

* Available wall and floor space for taps/transceivers.

* Areas within or outside of walls, ceilings, and floors for cabling, and repeaters/amplifiers.

* Dedicated rooms or floor space for network control units placed centrally or distributed appropriately.

* Floor loading.

* Adequate space for working on equipment; maintenance and service support.

* Availability of proper power, for normal and emergency operations.

10.8.2 Wiring (71)

Several types of wiring schemes are available for LANs; in making an appropriate selection, several factors must be considered:

* Is network intelligence needed?

* How much capacity is required?

* Are there long distances between the various stations in the proposed network?

* How important is data speed?

* Will the proposed network interface with other systems?

* What data error rate is acceptable?

* What are the projections for future expansion, relocation?

\subsubsection{Signal Amplification}

To ensure the quality of signals, amplifiers are often required. They are sizable units, and their placement should be planned for ease of access and aesthetic acceptability. There are two types of devices used for improving signals, the trunk amplifier and the line extender. The trunk amplifier is a sophisticated electronic device incorporating automatic gain control, automatic equalization, and noise suppression circuits. It can also serve up to four branch feeder points. A line extender has little sophisticated electronics and cannot serve feeders. 


\section{PBXs}

\subsection{FUNCTION}

The primary purpose of the PBX is to provide basic telephone service, divided into essential services and desirable options. Current systems are either based on centralized or decentralized architectures. Most systems are non-blocking (they are certain to acquire a path through the switch between two unoccupied ports). Traffic loading and holding time features of users do not limit the switch's internal capacity in the non blocking configuration.

\subsection{BACKGROUND}

About 60 of an organizations communication take place within one building or campus; another 228 is within 50 miles. The trend is toward digital data; which can be accommodated by a digital PBX. (72)

A PBX system has four basic parts; a switching matrix, control and user terminals and trunks. The PBX itself is comprised of the matrix and controls as well as line, trunk and service circuits that connect to telephones or other switches.(73))

While current systems are complex, their functions are almost identical to the manual switchboard where wires were connected to boards by means of jacks, to complete circuits. Lamps provided feedback information to indicate when service was needed, connections made, etc. The cords performed the same function that a switching matrix does in a PBX, while the modern dial tone serves as a surrogate for the operator asking for a telephone number. In both instances, a test is made to determine whether the connection can be made; i.e. if the line is busy, and if not, the call is completed. In both instances a feedback signal is transmitted when there is a problem and when the conversation is over. In the latter instance, the lines are disconnected in preparation for future service.

The trend in PBX's is toward distributed architectures, where switching is performed by an interconnected set of modules, which can be widely distributed; e.g. in different cities.

Maintenance and service support should be major factors in selecting a $\mathrm{PBX}$.

\subsection{CRITERIA FOR PBX SYSTEMS (74)}

* System diagnostics

* Least cost routing

* Call-detail routing

* A non-blocking digital switch matrix for heavy data traffic where holding times are long and eliminating switch load balancing over time. 
* Remote modules for multi-premise use

* Multiple processors to allow for system growth with expanded requirements.

* Speed/format/protocol conversion to house environments with different equipment and software needs.

* A high level programming language to facilitate customer control over system.

* Conformance with ISo network standards to avoid the need for tailored systems for particular applications and

hardware.

* Integrated voice/data terminals.

* PBX/LAN wideband communications channels between nodes and modules.

* Integrated advanced features; e.g. electronic mail

* Packetized data switching

\subsection{TECHNICAL ISSUES}

PBX's switch data in one of two modes: synchronous and asynchronous. Most can switch data asynchronously at speeds up to 19.2 kilobits per second, generally the limit of ASCII and PC's. In the synchronous mode they can switch up to the limit of the internal switching speed, 2 or 3 megabits per second. Organizations with low data terminal usage; less than 308 per day can use asynchronous communication with a PBX; others with more intense data requirements require other solutions.(75)

The advantage of a PBX for switching data is in the inherent design of the system. It is a concentrator; i.e. terminal devices contend for paths (time slots) through the PBX, which can support such usage because in the voice world, all terminals do not need paths simultaneously. Designs are most effective when voice and data traffic occur in short, bursty, low-speed data streams; requiring short holding times.

\subsubsection{Analog vs Digital Systems}

Analog transmission is a system whereby the pressure changes on a microphone or transmitter are translated into electrical currents proportional to the input signals. Signal amplification is critical because it is attenuated by cables. Unfortunately noises are amplified together with signals, until ultimately the message is lost. (73)

A digital system overcomes this problem because signals are recreated along the line with noise being eliminated each time they are regenerated. Voice signals can be transmitted digitally by means of pulses in successive time intervals which define the amplitude of the signal at any given instant. While many pulses are required, all the system has to do is detect whether or not a pulse is present, a simpler task than transmitting a complex analog signal. 
A digital switch enables data to be transmitted together with voice communications in an all digital network without the requirement for modems. However, most trunk systems are analog and transmission media such as coaxial cable can handle more analog than digital circuits simultaneously. Also, protocols among various systems and hardware are not standardized, and therefore data communications are made quite difficult.

\subsubsection{Integrating Voice and Data (76)}

Despite widespread advertising, integration of voice and data communications is still quite limited. For the most part they have been used to link ASCII terminals, transmitting asynchronously at 19.2 kilobits per second. (Synchronous methods are also used occasionally.)

A digital PBX is costly; up to $\$ 1000$ per user to line a special digital telephone into a 100-line digital PBX. (The cost is being lowered rapidly, and is dependent on the mix of voice and data requirements.) The cost includes telephone line cards with data transfer modules, telephones to link and format data for the PBX. This system is needed for simultaneous transmission of voice and data over the same twisted pair. (Some manufacturers use separate wire pairs for data and voice.) (77)

The popularity of personal computers provides a logical and economic use of PBX's, since microcomputers typically communicate asynchronously, in ASCII, at speeds under $9600 \mathrm{bit} / \mathrm{sec}$ through an RS-232-C Port. All voice/data PBX's support this connection easily, and there is the option of plugging the computer into the telephone line. Furthermore, personal computer users often use communication services for local processing, but the volume of use is light. The PBX is basically a switching system. Sharing lines among many users requiring periodic service, forms the basis for its design. Finally, the telephone, like the personal computer is likely to be on most desks in offices. It is available for use immediately, without added wiring or equipment required.

Each PBX system has a unique approach to integrating voice and data communications. Some digitize the voice at the telephone set and multiplex the voice and data together onto a single digital path into the PBX. Others use separate paths for voice and data from the telephone; voice traffic remaining in analog form, with digital data sent on a separate wire pair. The wires are then physically multiplexed within a single three or four twisted-pair jacket.(78)

The foundation of linking voice and data is the integrated digital telephone, with a RS-232C interface. The combined information is sent to and from the instrument in digital form, multiplexed on either one or two wire pairs. Each vendor has a unique proprietary architecture to handle communication between the telephone and PBX. In a typical multiplexed signal system, 
the instrument supports 64 kilobits per second of voice as 8000 samples of 8 bits each, and 64 kilobits per second of data in a synchronous mode, or 19.2 kilobits per second asynchronously, and 16 kilobit per second for control functions. Some PBX's can handle up to 2 megabits per second of data at the instrument by means of appropriate interfaces.

\subsection{ORGANIZATIONAL ISSUES}

One reason for the lack of progress in integrating voice and data communications is organizational separation of voice and data communications functions. Organizational barriers between the two are very strong, because of training, historical separation of activities, disciplines, etc. This separation is reinforced by the vendor community, who have few people equally knowledgeable about the two.

Voice traffic is generally of low call duration, with busy telephones used between 8-16\% of the time. Thus, a PBX switch with 500 connected instruments probably requires only 100 pathways. Data traffic tends to be longer (several hours is not unusual), especially when terminals are linked to hosts.

Network management functions for the PBX are:

* Accounting. Detailed records of usage.

* Restriction and Authorization. Class of service can be restricted to users.

* Maintenance. Monitoring and diagnosing of system operations minimizes system downtime and facilitates maintenance.

* Security. Information can be controlled; accessed only by authorized users.

An alternative to the self contained and operated PBX, is a system provided by the telephone company, supplied from a central office - Centrex. It lacks some user control, integral to the PBX such as voice forwarding and data switching.

If communication is central to the major function of a large organization, then a PBX is called for. When less than 50 users are involved, Centrex service offers many advantages. Smaller companies are often unsure of their future needs, and systems can be rented and running in less time than it takes to plan, purchase and install a PBX. Centrex also provides the opportunity to add an unlimited number of lines without major capital expenditures.(79) 


\subsection{FUNCTION}

To prevent life and property from harm or loss caused by accidents and natural or man-made events. Life safety systems include fire protection equipment and systems such as sensors and alarms, extinguishing systems, exit lights, control of emergency exits, emergency lighting, control of fire dampers, smoke exhaust and ventilating fans, and some aspects of site security.

(See Figure 23.)

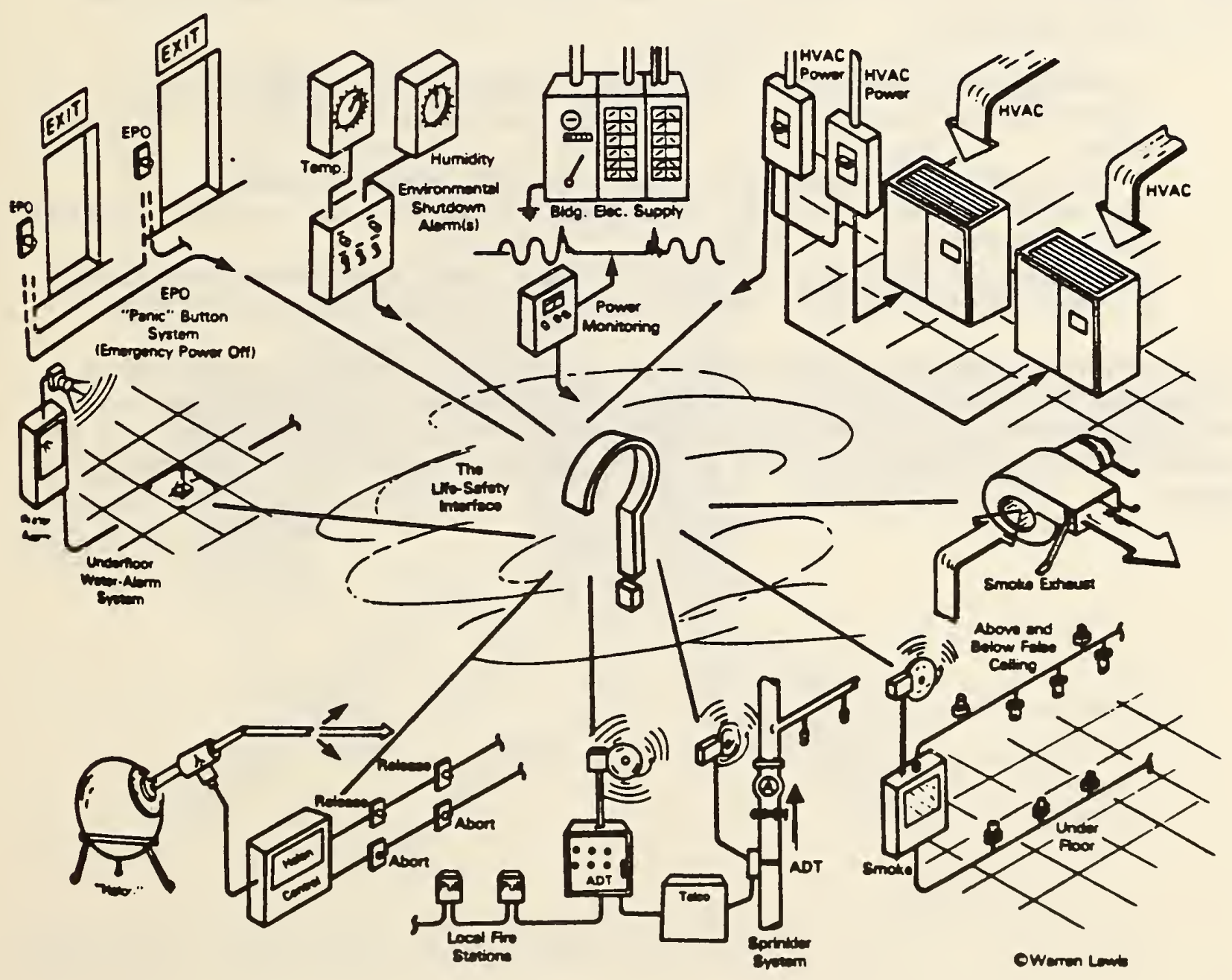

Figure 23. Life Safety Interface Elements 


\subsection{CRITERIA}

* Ensure safety of building occupants.

* Minimize equipment and data loss during emergencies.

* Minimize "downtime" of operations after emergencies.

* Establish adequate safeguards against system activation when not required.

* Systems should be easy to operate and monitor.

* Power should be independent of main system.

* System design should facilitate future expansion and/or changes.

\subsection{TYPICAL APPROACH}

The design of a life safety system can be accomplished by the following steps:

* Develop a list of life-safety subsystems, individual devices and controls, then list the operating modes of each one.

* Develop a strategy for linking and prioritizing operations, showing which systems trigger, or prevent others from operating.

* Resolve time delays, inhibitions, and sequences necessary to eliminate logical conflicts and establish priorities.

If these systems $c$ an be placed in a single, readily accessible cabinet, and the circuits clearly documented for installation, maintenance and future additions, many potential problems can be avoided. A possible system configuration appears on the next page: 
ADP site basic life-safety elements to be coordinated include: Athe halon fire suppression control system cabinet; B--the smoke, fire and sprinkler alarm and arming system cabinet, C--a

"console" to monitor other environmental parameters such as room temperature, humidity, water under the floor, status of power sources, HVAC equipment, etc. (See Figure 24.)

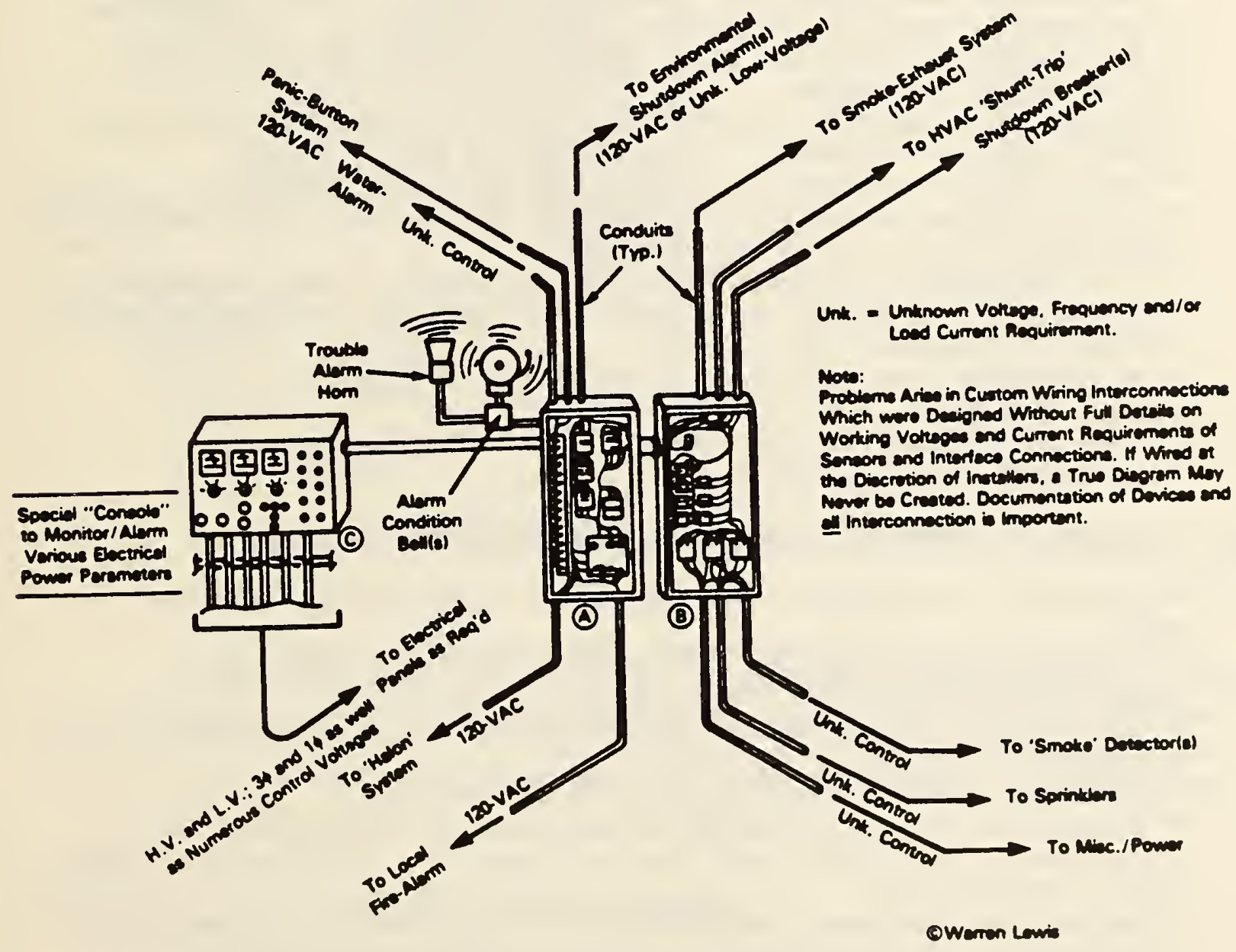

Figure 24. ADP Site; Life Safety Elements 


\subsection{CHECKLIST-LIFE SAFETY SYSTEMS}

Systems should have the following features:

* Smoke detectors based on optical or ionization designs.

* Temperature and rate of heat rise detectors.

* Flame detectors.

* Halon discharge control units accepting inputs from sensing alarms. They supervise systems, produce audible alarms, provide manual activation and manual override, time delays and equipment shutdown; finally, they suppress fires using halogen gas.

* "Panic" button for turning off power.

* Automatic shutdown of: central computer, HVAC system.

* Automatic startup of smoke removal fans.

* Automatic control of fire dampers, blocking air ventilation passages.

* Controlled personal access to central computer and life safety control system.

* Manual and automatic initiation of alarm systems.

* Fire related circuits should run separately in conduit containing only those conductors.

* A data logging system identifying significant changes in systems; the ability to access information concerning status of system components.

* A schedule for testing primary and backup life safety power systems.

* Continuous monitoring of on-off condition of major equipment and systems.

\subsection{CHECKLIST-CENTRAL CONTROL OF LIFE SAFETY SYSTEMS}

* Location convenient to personnel expected to respond to them.

* Controls and displays grouped according to major

functions, e.g. fire, HVAC, power.

* Consistent grouping of system elements, e.g. locations of displays, controls.

* Clearly labeled indicators and controls.

* Schematic diagram should be available, including "as wired" and "as installed" corrections for all devices and interconnections.

* Formal acceptance tests should be conducted; scheduled maintenance and testing to verify system performance typically annually or semi-annually

\subsection{ENVIRONMENTAL CONTEXT}

The design of life safety system must be integrated with the other power, electrical and information systems in a building. For example in a building containing energy management and advanced telecommunications systems, monitoring and control activities might be centralized with life safety systems. 
Following is an illustration of a life safety system control configuration:

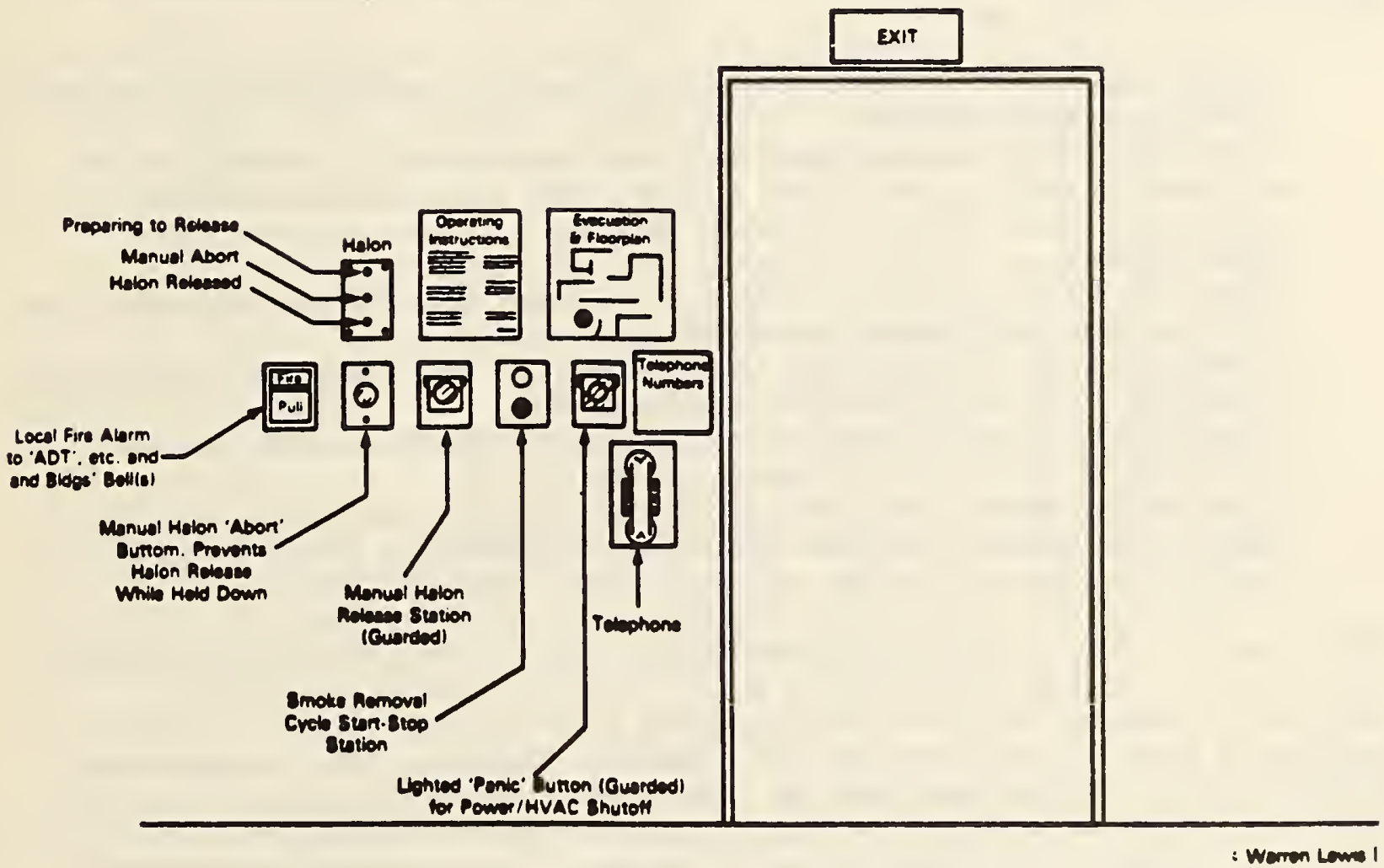

A well-organized, convenlent, and coordinated arrangement of 11fesafety Indicators and controls: An emergency telephone wh th separate IIne (not via PBX or switchboard) 18 needed near the exit and tbe I1fe-safety Indicators and controls. The audible alarm should not be 80 close to the telephone as to defeat attempts to comunicate.

Figure 25. Life Safety Control System 


\section{INTELLIGENT BUILDINGS- INTEGRATION OF COMMUNICATIONS AND AUTOMATION SYSTEMS (80)}

One definition of intelligent buildings is: "communication between people to people, people to computer, and computer to computer". Another commonly employed characterization is where building services consist of several integrated and interactive systems- e.g. building control, information management, and telecommunications. The systems are served by a single wiring system.(81) For example:

* The information management system can automatically route data to a workstation.

* The building management system can detect a fault in an air conditioning unit and direct the telecommunication system to send an electronic message to the maintenance supervisor.

* An identity card can admit a person to a building, turn on lights, and activate the HVAC system.

* During a fire, systems can pinpoint its location and alert building and fire station personnel.

* Buildings can be monitored for around the clock security by sensors and/or or closed circuit tv.

* Microprocessor controlled elevators can be programmed to take coordinated action, thereby responding to peak load conditions by reducing waiting time for occupants.

Very few, if any, buildings can claim this level of integration.

The basic premise of the intelligent building is that it will provide a better environment to conduct business by integrating building and office systems by advanced communications. The major benefits of such integration are greater functionality to the tenants, lower installation and operating costs, and potential benefits through the resale of services. (82)

Intelligent buildings are often designed with the aid of computer aided design (CAD) systems. The workplace now has a combination of terminals, telephones and cables with responsibilities for systems scattered throughout the organization. Barely controlled chaos is the result when rearrangements of workstations are needed.

\subsection{BACKGROUND}

An integrated system may be less costly to install, operate and maintain than separate systems due to the elimination of redundant communications networks. It will provide one communication path for voice and data signals. Also, since one contractor will develop the system there will be less coordination needed and fewer individual maintenance contracts.

Integrated systems are built around a stand alone microprocessor that monitors and controls various functions. Microelectronics 
makes it possible to create a unified, centrally managed system. A voice/data network can connect all the systems, while linking the building to the outside world. Central computers and microprocessors tie every part of the building together. The network has electronic monitors and direct digital controls for all of the building equipment. All building functions can be monitored on computer terminals from a central location. Building can operate automatically but remain under the control of a human operator.

Economic viability of such systems has been demonstrated for buildings of 300,000 square feet or more. Greater functionality occurs as a result of greater tenant control over comfort and safety and additional services. Among the potential benefits of such systems are:

1. A single cabling system can reduce wiring costs by $30 \%$ over traditional methods.

2. The number of support processors can be cut by $25 \%$. 3. Operating costs can be cut through reduced manpower, space and energy, with savings up to $20 \%$. 4. Maintenance savings of up to $20 \%$ are possible because of less equipment.

5. Energy savings. The telephone system can be used to control lighting and HVAC during off hours. Tenants can be billed for actual costs.

6. Security monitoring. Controlled access and intrusion detection systems can be monitored centrally.

7. Critical equipment such as data processors can be monitored on a 24 hour a day basis.

8. Automatic fire and smoke detection and control.

There are various levels of system integration, based on the design elements combined:

* Non-integrated. Each system has a stand alone processor and dedicated cabling.

* Shared cabling. The minimum level of integration involves separate processors and sensors or terminals with shared conduit and/or cable.

* Interfaced sub-systems. Separate, stand alone processors, actively interfaced can share data and communicate with one another. Cabling may or may not be shared.

* Total integration. Shared processor(s) and cable highways.

Several functional levels of integration have also been defined: (83)

1. Energy management, life/safety, and security systems

2. Conference/meeting room space; photocopying, word processing centers. 
3. Telecommunications, services including long distance voice and some data; telephone service using a building core cabling system.

4. Sophisticated office automation and high speed communication and data processing; wideband communication for video conferencing.

\subsection{CRITERIA FOR INTEGRATED SYSTEMS}

* Integration of energy management, HVAC system operations and maintenance, data acquisition and management, fire and smoke management, and system alarms.

* Air handling system, fans, duct smoke dampers, outside air, and exhaust dampers integrated with fire and smoke controls.

* State-of-the-art sensors.

* Distributed building automation system with stand-alone field interface devices.

* Direct digital controls, as appropriate.

* Conventional/adaptive algorithms for HVAC system controls.

* Fire system overrides other systems when necessary; it is always operational and secure from interference.

\subsection{COMMUNICATIONS}

With the breakup of AT\&T, end users have many choices and are ill prepared to make them in a cost beneficial manner; e.g. countless systems are available and hundreds of vendors are selling products for telephone service.

The telecommunication system switches and routes all voice and data within the integrated building. It also connects internal sources with the outside world. The PBX is used to route signals originating in any of the building systems; e.g. a control signal from the CPU of the management system to a fan motor; data between a PC and the CPU. Telephone systems can provide speed dialing, the ability to serve as an intercom, display messages and conduct conference calls. For long distance calls, the PBX can automatically select the least expensive routing path.

The capability of integrating communication, monitoring and control systems reflect newly emerging technology which has only been tested in a few applications. Each major function is likely to have its own stand-alone processor with individual function multiplexers connected to them. Subsystems which should be considered for inclusion in an integrated network include:

* Voice and data communications.

* Office automation (and networking).

* HVAC, security, fire safety.

* Elevators.

* Lighting. 


\subsection{WIRING SYSTEMS}

The problem of distributing signals within buildings still is unresolved; i.e. how should signals be brought from the builaing boundary to and from end-users through common areas. Often signals must be transmitted over wires with different characteristics. In order to cope with this new situation, several design and construction issues require attention:(84)

1. The capacity of feed conduit, vertical risers, telephone closets, telephone switching rooms, and tenant conduit. 2. Electricity. Is there sufficient power for the installed and planned automated equipment and standalone environmental systems; e.g. local HVAC units. Is the quality and reliability of power appropriate?

3. The roof. Is it sufficiently strong to accommodate antennas. Do risers extend to the roof?

4. Ceiling heights. Will the plenums accommodate cabling and ducting needs? Is there space for raised floor designs? 5. HVAC systems. Are they sized for the new equipment? Are there spaces for the standalone systems?

6. Are wiring diagrams updated to reflect changes?

7. Is it applicable to the widest possible array of tenant needs?

8. Modularity. Is the design a modular, cost-effective mieans of implementation to minimize cost and aisruption of ongoing work?

9. Can the system adapt to changing technologies readily and at minimum cost.

\subsection{MECHANICAL SYSTEMS}

Energy management systems are designed to maintain a comfortable and safe environment with efficient energy usage. Sensors monitor temperatures throughout a building, with information being transmitted to local microprocessors, which adjust fans and dampers. Each area of the building can have the local temperatures adjusted to the occupant's desires. The system can account for usage by curtailing heating and air conditioning when spaces are not occupied. During peak power usage periods, when energy prices are higher, building temperatures can be lowered in the winter and raised in the summer, yet kept within comfortable levels. Sensors can be used to turn off lights when rooms are unoccupied. Energy management systems to control light and HVAC are important elements of intelligent buildings, but they must be manned by people who fully understand how to operate them efficiently.

The major impact on mechanical systems has been a move away from constant air volume (CAV) systemis to variable volume (VAV) systems. The reason for the change is to increase flexibility and control. Conventional (CAV) multi-zoned systems are usually limited to 10 to 12 zones per flow; with varjable volume systens, 
the number is unlimited. Air conditioning flexibility is also important especially when limited data are available concerning how many new computers will be provided for an organization. One design approach to accommodate new equipment is to allow room for additional cooling capacity when the load requires it.(85)

HVAC systems can adjust to changing environmental conditions and to different internal requirements. For example, an office being warmed by sunlight will be conditioned differently than an interior office. Systems can keep track of exterior and interior air temperatures and use outside air for cooling when conditions warrant this approach.

\section{6 FLOORING SYSTEMS}

Other design approaches have been increased plenum depth from four to at least five feet and pedestal flooring anywhere from 6 to 24 inches. The larger plenums accommodate overhead cable trays that typically run above corridors and serve computers, peripherals and telephone systems. The pedestal floors were originally used for mainframe computers but are now employed for general cabling requirements in many buildings because of their easy accessibility.

\subsection{CASE STUDY}

The intelligent building is part of an evolutionary trend that in the 1970's focused on energy management systems. One such

approach is the use of shared tenant services. The primary market for the shared tenant services are tenants occupying less than $100,000 \mathrm{sq} f t$ of space; three floors or less of a major office building. (86)

The service provider owns the common equipment, processors and terminals; tenants subscribe by paying a monthly fee for services, include voice and telecommunications systems, work station networking, PCs, electronic mail. and filing, customer training, consulting, on-site repair, operations. (87)

City Place in Hartford, Connecticut exemplifies this approach. It has an optical fiber cable used throughout the 38 story building.

* It is equipped with infra-red sensors which are used to detect the presence of people; when spaces are unoccupied for 12 minutes, the lights are automatically turned off.

* Fire sensors ring an alarm, notify the fire department, pressurizes the floors above and below the fire floor and exhausts the smoke from the fire floor.

* A Lexar digital telephone system is used to transmit data and voice information simultaneously. It can be used to transmit reports from one workstation to another and uses a least cost routing scheme for long distance telephone conversations; lines are bought by united Technologies from major carriers and the service is resold to tenants. 
* Tenants can lease computer hardware and software.

Merrill Lynch, a major tenant in City Place, has its own system because it couldn't take the chance of sharing telephone service; most of its business is transacted by telephone and availability of lines is essential to its operations. Internal control of its own network is paramount.

\subsection{CHECKLIST FOR SYSTEMS INTEGRATION}

* Which functions are integrated/controlled?

* Which functions can or must be controlled separately?

* For each major integrated function, what operations are controllable, and what features of control, programmability, input/output, display, etc. are available?

* What is system architecture, i.e. central/distributed; redundancy, sensor types, conductor media?

* How does the system assure reliability?

* What is done to protect functions, zones, the entire system?

* What manual backup systems are available?

* Who provides emergency repairs; how long will it take?

* What are initial and operating costs of system (including maintenance and repairs)?

* Does the system require dedicated staff to monitor and operate it? If so, what training and experience is needed? * What are special physical requirements of the system, e.g. size of central and distributed processors, control panels, HVAC and power, cable runs?

\subsection{INTELLIGENT BUILDING MANAGEMENT DECISIONS}

Among the questions to be answered by tenants and facility managers are:

* How much growth is anticipated in the next 5,10 years?

* What are the initial and long term costs of technology and design implementation?

* How reliable are the technologies and systems?

* What are the cabling system requirements for voice, data, video?

* What is the viability of shared tenant services? e.g. what are costs/benefits; what support personnel are needed; should it be done in house or managed externally? 
* The integration of technologies in existing organizations and their assimilation by users in their daily work.

* How to include technological factors in the design process.

* Office design based on system concepts rather than focusing on micro issues such as individual hardware components.

* More new and small firms are being formed; they often are more innovative than larger ones and more likely to employ the latest technologies.

* Larger organizations are delegating more decision making to subordinate groups; technology is fostering decentralization. They are also providing less technical and service support. * Building designers are being asked to provide support such as health and athletic center, day care facilities, etc.

* The ratio of professional and staff members is changing; fewer support personnel are employed.

* Advanced telecommunications has enabled companies to disperse operations geographically; small suburban centers are becoming popular in contrast to large centralized operations.

Table 26. SMART BUILDING CHECKLIST (89)

\section{PROJECT BACKGROUND}

1. Who is considering enhancing the structure? Why?

2. Are the structure and tenants good candidates for

building enhancements? If so, what criteria used to determine?

3. What support services are currently available to be shared?

\section{BUILDING STRUCTURE}

1. What wiring technologies are used within building?

2. What provisions are made for future enhancements?

3. How will technology changes affect the support systems, e.g. HVAC.

4. What flexibility is available to tenants who move or require added space?

5. How would the cost and design of the following support systems be affected by proposed enhancements?

* Electric power, air conditioning, fire protection

* Direct design and materials costs; e.g. space, ceiling heights, vertical risers, floor ducting, space for equipment, floor loading.

* Wiring system specifications, e.g. conduit, cable

* Security and alarm systems.

6 . What are design and coordination tradeoffs between retrofit and new design?

7. What provisions are there for tenant service expansion?

8. What specific design aspects of the building (other than wiring) are affected, e.g. satellite dish? 


\section{EQUIPMENT TECHNOLOGY}

1. What are technical specifications of the PBX switch. e.g. maximum capacities of trunk groups, consoles, class of service?

2. Is the existing tenant equipment compatible with the proposed system?

3. How were the tenants' communications needs determined?

4. What voice and data features are available with the PBX?

5. What types of data and word processing equipment will be used? What other systems will be employed?

6 . What communications systems will be used; local area networks, microwave, satellite, etc?

7. What specialized common carrier services are offered? How are they accessed?

8. Does the building owner have the option of specifying and owning cabling systems and control, if the vendor's products do not perform properly.

9. How is the PBX upgraded to access new technologies?

10. What is signal distribution system; how does it handle multiple local area networks, different data processing protocols? Will the tenants require a separate cable plant to handle voice and data systems?

\section{SYSTEM MANAGEMENT}

1. Is there a detailed plan for systems maintenance?

2. How can tenants subscribe to partial services, to complement equipment and services already owned.

3 . What are the backup services available to deal with system malfunctions?

4. What centralized monitoring and control functions are available? Who performs functions? Provide details.

Table 27 describes the possible design impacts of integration and telecommunication systems. 
Table 27. Integration and Telecommunications Systems- Possible Design Impacts (43)

\section{ISSUES}

INTEGRATION SYSTEMS

- Volce/data Interface

- Security

- Electrical

\section{- Fire-safecy}

\section{Environmental}

\section{PRESENT OFFICE}

Separated

Stand-alone

Decentrallzed

Separate bystem

Separate heating and NC syotems

Stand-alone volce

Decentral1zed, standalone

Dedicated Telephone IInes

$$
\text { and external }
$$
colcunications oyeteas

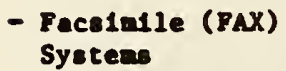

- Types of Cable

\section{AUTOMATED OFFICE}

Integrated using state-of-the-art equipment

\section{Integrated \\ Centrallzed and Integrated \\ Integrated with
"Central nervous
Systems}

Energy management system

Combine volce/data
(D1gital PBX's)

Centrallzed, Integrated

Hybrid (satellite, alcrowave fiberopi1c, and telephone 11nes)

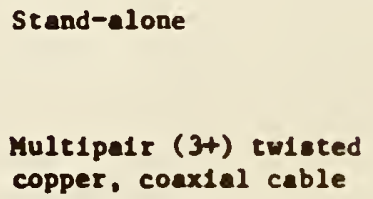

A network 1ntegrated

Large capacity fiber triaxial
POSSIBLE IMPACT

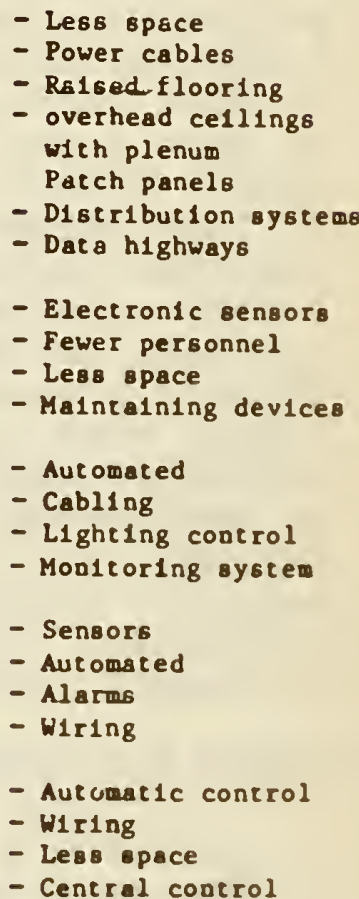

- Different cabling requiresent

- Less equiprent roon space

- Lees equipent

- Network laplicatione

- Comblned with other equipont eyeteas

- Cabling requirenent.

- Interface

- Interoperab1l1ty

- Equipont definition

- Structure requirement.

- koof antenna.

- Cabling

- Site curveys

- Space for concentratore

- multiplexors, etc.

- Interfaces

with other aystems - Cabling

- Environaent conelderation

Rad 10 and/or Infrared Multinedia in elngle jacket.

Twated fiber; $\cos x$ replacenent
- Increased ize, protection of equipeent in closet

- More space needed for closers, vert $1 \mathrm{csl}$ cable diectibution 


\subsection{NATURAL AND SYNTHETIC FABRICS (27)}

\subsubsection{Function}

Aside from its other practical and aesthetic features, a major purpose for the use of fabrics is to contribute to the desired acoustical performance of the automated office. Fabrics are used extensively for floor coverings, furniture and panel systems, as a means of controlling noise and enhancing auditory privacy.

\subsubsection{Criteria - Checklist (Fabrics)}

* Permanency. Will the fiber content fade in the sunlight or disintegrate with age?

* Moisture and mildew resistance. Does the weave allow dimensional stability for dry and humid conditions? Will dampness promote mildew or outgassing of chemicals? * Maintenance. Is it resistant to abrasion? Can it be washed, dry-cleaned or refinished?

* Fire resistance. Is it flameproof? Will it melt and produce dangerous flames and/or fumes?

* Installation. Is it easy to work with? Can it be easily replaced?

* Thermal resistance. Will it expand or contract with thermal absorption?

* Weight. How heavy is the material? Can it be easily handled?

* Sound absorption. What are its characteristics with respect to absorbing, dampening, reflecting sound?

* Appropriateness. Is it consistent with the uses of the space?

* Availability. Can the material be obtained for replacement and expansion purposes; for how long?

* Decorative palette. How varied are the colors and textures?

* Price. Is it cost effective?

* Aesthetics. Is it attractive?

* Electrostatic buildup. What are electrostatic features? Table 28 presents the positive and negative features of
carpet materials. 
14.1.3. Carpet Material Characteristics

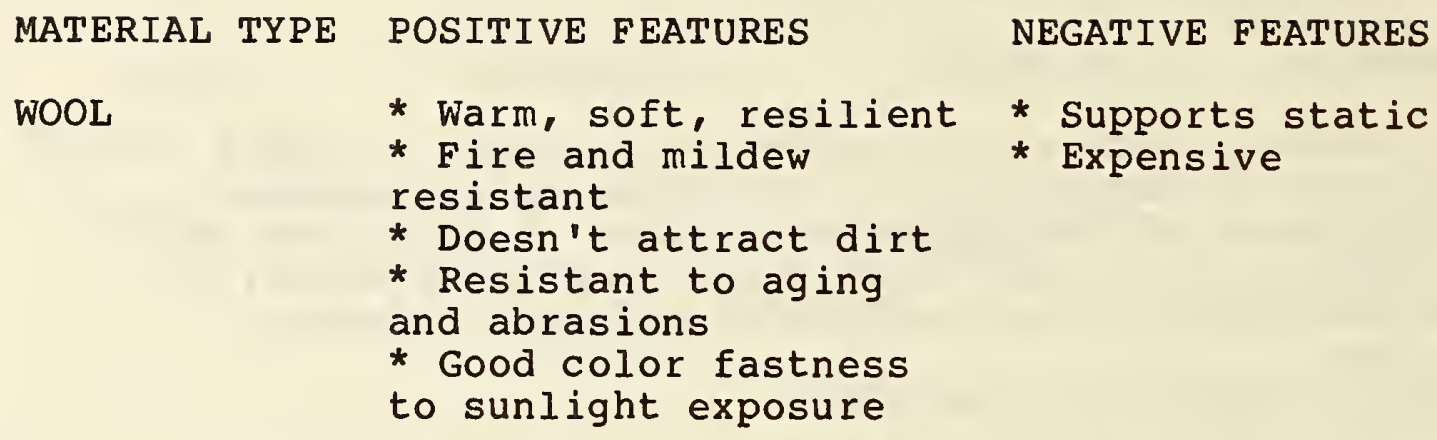

* Warm, soft, resilient

* Fire and mildew resistant

* Doesn't attract dirt

* Resistant to aging

and abrasions

* Good color fastness

to sunlight exposure

* Supports static

* Expensive

NYLON

* Very tough material

* Good static control

* Resistant to mildew

* Resistant to aging

and abrasions

* Blends with other

fibers

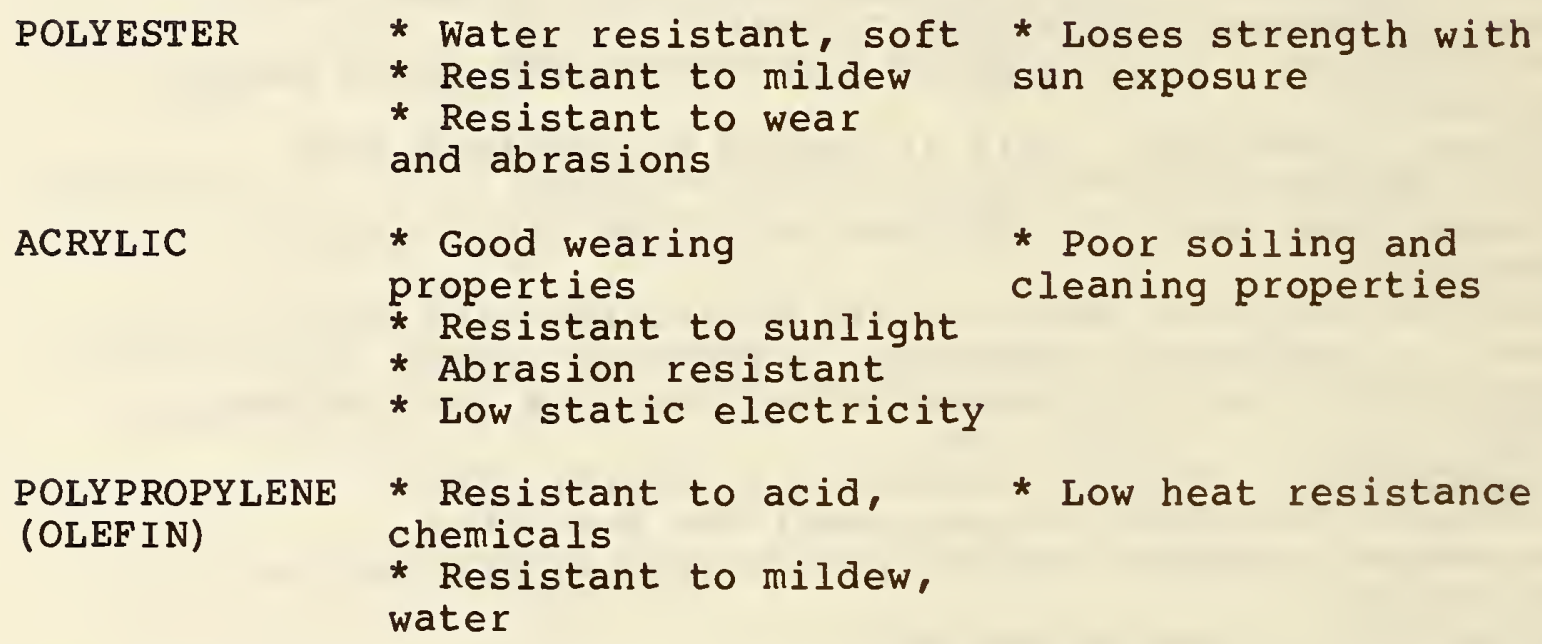

ACRYLIC

* Good wearing

properties

* Resistant to sunlight

* Abrasion resistant

* Low static electricity

POLYPROPYLENE (OLEFIN)

* Loses strength with sun exposure

* Melts on contact with flames

* Attracts dirt 


\subsection{CARPET TILE}

Office automation has led to the increased use of carpet tiles for floor coverings. Their modular design provides underfloor accessibility, easy maintenance and replacement consistent with the need for flexibility.

\subsubsection{Criteria}

The following performance features are important when carpet tiles are employed:

* Abrasion resistance and wearability.

* Appearance retention; the ability to withstand crushing, fading, soiling and maintenance.

* Proper maintenance.

* Appropriate combustion and smoke performance characteristics.

* Inhibiting the accumulation of static electricity through built-in special fibers, wires or coatings.

* Cost-effectiveness as compared with the materials and maintenance cost of alternative choices.

* Maintenance of critical performance features after initial installation, e.g. loss of adhesion.

Figure 26 shows typical static voltages generated by walking
on common floor surfaces. 


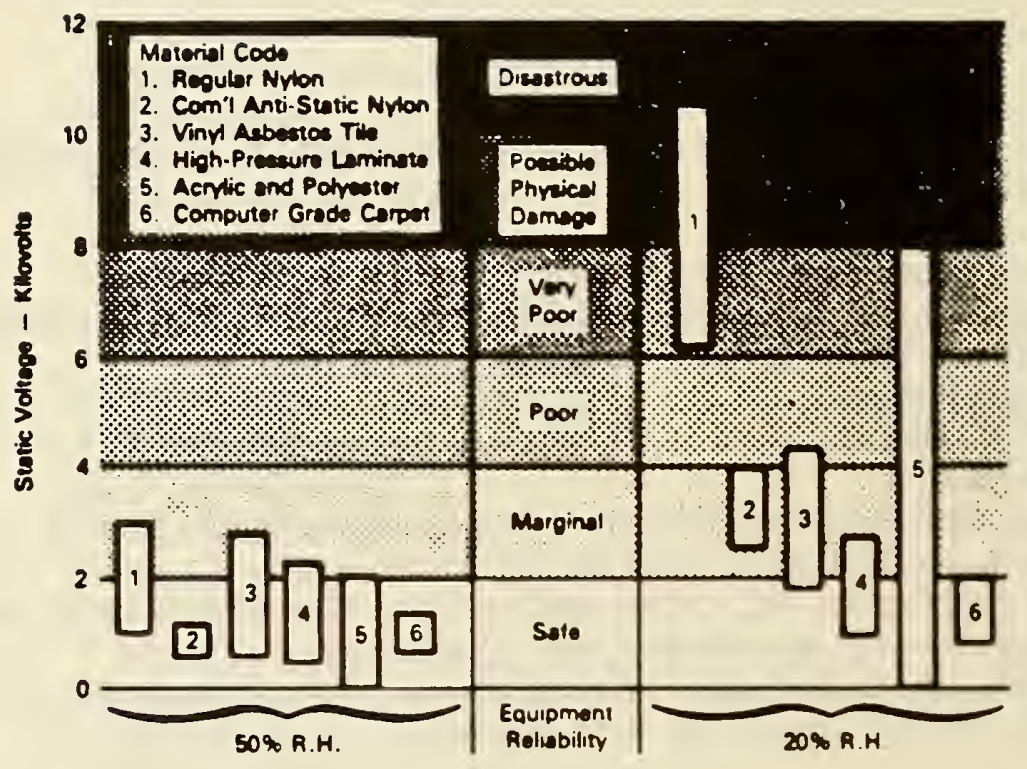

Figure 26. Typical static voltages generated by walking on common floor materials (18). 


\subsection{OVERVIEW}

A comprehensive literature search, a questionnaire survey and a roundtable meeting formed the basis for the information in the present document. These activities, augmented by interviews with architects and other professionals, resulted in identifying specialized spaces which merit consideration in designing for office automation (91).

* An atrium. This can be used to offset the technological emphasis of automated offices by providing a connection with the outside, a change in scale, and a place for social contact.

* Training and media presentation rooms. Organizational and technological changes occur so rapidly that a continuing need exists to upgrade personal skills and transmit information to staff members.

* Meeting rooms. With the popularity of open-space design, fewer spaces are available for private meetings of small groups. Such spaces, and those accommodating more people are required to facilitate staff interactions, and offset the isolation inherent in many VDT-based activities.

* Centralized reproduction facilities. New and sophisticated systems provide the capability to produce documents with the quality formerly available only from printers. The cost of such systems can only be justified by organization-wide use. These systems are usually quite noisy and should be acoustically isolated from the general work floor. Merging of computerized printing, image processing and reproduction facilities is a recent development.

* Centralized information storage. For the foreseeable future, paper will continue to be an important media for storing information. In addition, microfiche, magnetic tape and disks must be stored. The latter two types of storage require careful environmental control.

* Centralized communications and information processing. More offices are likely to contain CPUs and systems such as telex and facsimile reproduction. Such equipment require specialized spaces and facilities for occasional users.

* Decentralized computer/controller facilities must be located throughout the building to satisfy future cluster controller and/or standalone CPU requirements.

* Equipment areas. As more technology is moved into the office, more spare parts, cables, replacement systems and 
facilities for maintenance and checking are all needed. The demand for these resources will accelerate as office activities depend more on automation, equipment outages being very costly to productivity.

* Equipment staging areas are required.

* Clusters of offices, grouped according to similar activities.

* Furnishing storage. With the popularity of open-office design, the increased acceptance of ergonomically responsive furnishings (tailored to individual needs), and the need to respond rapidly to change, there is a substantial need to maintain an inventory of furniture, panels, etc.

* Convenience rooms for copiers, printers, etc.

* Magnetic media storage. Careful environmental control is required to ensure that information is preserved. Warping and stretching occurs in hot environments. Low humidity can cause problems due to static buildup, allowing dust and dirt to accumulate. Variable environmental conditions can affect the longevity and reliability of media performance. Among other potential problems are:

- Magnetic fields. Media should be kept away from transformers or large motors that generate magnetic fields. (This might influence the placement of UPS systems.)

- Shock and vibration. These conditions can cause discs to lose information.

- Chemical solvents. Volatile chemicals can degrade media, and should not be stored nearby.

\subsection{TELECONFERENCE FACILITIES}

\subsubsection{Function}

The high cost of travel and the proliferation of organizational meetings has increased the demand for the capability to conduct meetings remotely. The technology required to accomplish this has been available for some time, with costs being the major obstacle. Technological advances have resulted in substantial economies and increased communications capabilities, and it is anticipated that teleconferencing facilities will become more commonplace in the near future. Special presentation skills and meeting formats are required for successful teleconferencing.

A teleconference is a meeting involving two or more people at two or more different locations, with discussions being transmitted through audio, digital, and/or video techniques. Video teleconferencing offers the greatest potential for substituting 
for meetings because of the availability of powerful technologies to simulate a live meeting.

\subsubsection{Criteria}

* The room should facilitate the communication of verbal and visual information and reflect the design needs of the system vendor.

* The space should be free from visual and noise

distractions.

* Required support equipment should be readily accommodated without impairing communications.

* Room decor should be consistent with normal work areas major users.

\subsubsection{Environmental Context}

* The room location should be suitable for the expected users. It should not be part of an executive suite, unless top management are its primary users. Middle managers often make the most effective use of these facilities.

* The room should be located away from mechanical devices such as elevators, central air conditioning and heating, major systems such as PBX's and computer rooms, and from street and office sounds.

15.2.4 Support System Considerations

15.2.4.1 Acoustics

* Existing rooms are often converted to teleconference facilities, creating acoustical problems in some instances. Most such rooms are constructed of standard building materials that are 4 feet by 8 feet rectangles. These dimensions often lead to a room designed with a ratio of length of width to height of $3: 3: 1$, which reinforces many negative acoustic properties such as reverberation. A ratio of $5: 3: 2$ is more preferable. A still more effective way of reducing acoustical problems is to use non-parallel walls and/or having large pieces of furniture in the space to scatter sound.

* Careful acoustical treatment of all surfaces, walls, ceiling, and floors is required in such facilities.

* The noise from the support equipment can be minimized by locating HVAC equipment required for teleconferencing in acoustically isolated adjacent spaces.

\subsubsection{HVAC Equipment}

In a fully equipped room containing facsimile systems and VDT equipment, provisions must be made for proper ventilation and for the air conditioning equipment. 
* Because of the need to see people and visual displays clearly, lighting and color selection for the room are of critical importance.

* Room surfaces should be non-specularly-reflective. The colors selected, including those of carpets and furnishings should have neutral tones.

* The system vendor should participate in the decision process for making lighting and color selections.

\subsection{DATA CENTER}

\subsubsection{Function}

The data or computer center houses the mainframe computer and supporting equipment in large organizations.

\subsubsection{Background}

A controlled environment is the major goal in planning and designing data centers; rooms must be dust free, cool and antistatic. Fluctuations in power, temperature, humidity, or air distribution can cause severe problems. The room should have compartmentalization of functions when possible; e.g. printers in separate rooms because they do not have same environmental needs.

The center must have adequate and reliable power sources; the site should accommodate a flexible communications network, including land lines, line-of-sight microwave, and satellite facilities. Construction activities near the site can be disruptive; e.g. severed power lines, power fluctuations, disruptions of power as well as dust and broken water lines.

The computer room is the heart of the center. Its size depends on the system employed. The design must have adequate space for current needs and provisions for growth and expansion. This can be accomplished by locating staff lounges, maintenance, repair and storage spaces nearby. These facilities can be relocated when systems are upgraded and two systems must be colocated to avoid shutdown of operations. (93)

The computer room should have a raised access floor from 15-18" in height to accommodate cabling and air plenum for direct cooling of equipment. Electronic storage is usually adjacent to the computer room. If the data center relies heavily on phone lines for data transmission, a separate telecommunications space is required to house equipment. If data are generated in-house, a data entry space must be provided. 
* What is the age and general condition of the building?

* What is the occupancy schedule?

* What will be located above and below the data center?

* Will the building be owned or leased by the organization?

* Is there a freight or service elevator; is it large enough to carry needed equipment and supplies?

* Is the clearance between floors adequate?

* Are the load-bearing columns adequately spaced for center?

\subsubsection{Criteria for Computer Room Design (95)}

* Visual security from main streets

* Controlled access to CPU

* Windowless for security and to minimize moisture infiltration and exfiltration

* Closely regulated temperatures, humidity and air quality

* Process cooling to accommodate equipment

* Vapor sealing on ceilings, walls and floors to maintain proper humidification

* Served by two independent electrical power sources

* Located away from major microwave transmissions

* Backup and/or UPS

* Circulation corridors around manned and unmanned machine rooms for added security

* Unobstructed flexible open area with expansion capabilities

* Floor-to-ceiling height sufficient for raised access floor

* Support systems located apart, acoustically isolated when needed

* Floor drains

* Fire detection and suppression system

\subsubsection{Air Conditioning}

Clean, warm, moderately moist air is needed in data processing centers to assure optimal computer, peripheral and memory performance and longevity. Air conditioning accomplishes two purposes:

* Provides cool air mainly by removing heat:

- produced by electronic hardware

- transmitted across room boundaries

- produced by people

- brought in with ventilated air

* Provides latent cooling, which condenses water vapor from the humid ventilating and infiltrating air, and moisture generated by occupants.

Computer room air conditioning systems are primarily "process cooling" type systems; human comfort is not the primary concern. Considerably more air must be circulated through cooling coils 
than in traditional systems. Special humidity requirements make the introduction of outside air undesirable. (96)

Process cooling systems maintain humidity, temperature and air cleanliness control. Most such systems are direct feed types, pumping air directly into the computer room through the cavity beneath the pedestal floor, which provide space for the cooling units, processed air, and cabling. Vapor sealing of floors, walls and ceilings minimizes moisture migration from outside and inside computer areas, ensuring proper humidity control.

Computer equipment requires year round cooling with close humidity and temperature control. Prepackaged downflow air conditioning units with integral automatic controls should be used to maintain uniform temperatures $(72$ degrees $F,+$ or -2 degrees) with relative humidity of $50 \%$ (t or -5 percent). Domestic cold water is needed to each air conditioner for humidification. Redundant air conditioning units should be available as backup for working units.

\subsubsection{Electrical Power}

Electrical power can seem to be an uncontrollable resource; the power quality delivered to a site must be used, whether supplied by a private or public utility or self generated. Riding along on the electrical wave are noise spikes, surges, caused by lightning, switching transients, or other equipment working nearby. Brownouts and total loss of power are other dangers.

Clean electrical power is essential and backup power and UPS systems are both used to guard against outages and fluctuations. The best design is to have dedicated power to the center. When backup power and/or UPS systems are used, space is required to house equipment. A 3-phase, 5-wire power distribution should be provided. Average power loads range from 25 watts/sq ft to 50 watts/sq ft. Power requirements differ significantly from system to system; varying from $50 \%$ to $100 \%$, depending on the manufacturer. "Less expensive" computers often cost far more in the long run, when taking energy usage into account.

\subsubsection{Electrical Power Checklist (97)}

1. Importance of trouble free operations of center.

* How much down time is tolerated; length of each, frequency?

* What can interrupt ADP power; e.g. HVAC plant?

* Are operations dependent on communications lines requiring continuity of power? 
2. Site location problems- is site subject to:

* Natural hazards- storm damage, winds, snow

* Man-made hazards-vandalism, sabotage, vehicular

collisions

* Offsite damage to communications and power

* Are building and/or electrical codes overly restrictive?

* Are there backup distribution lines in case of failure of main one?

3. Quality of power.

* Review power outage and disturbance records of utility.

* Ask other major users about power quality.

* Plan to have HVAC equipment power separated from ADP power.

\subsubsection{Fire Detection and Suppression}

The data files should be properly protected with the latest fire and security systems. Speed is critical and state-of-the-art detection and suppression devices are worthwhile. Ionization or photoelectric detectors in the ceilings and underfloors are preferred because of their sensitivity to fire conditions and the capability to automatically trigger fire suppressants and alarm signals. Halon based systems are very effective because of their speed and minimal damage and disruption to computer systems. The alarm systems should be interconnected to a central system.

Fire extinguishing agents such as water and chemicals can cause extensive damage to sensitive electronics and magnetic storage media. Irreplaceable program documentation and business can be destroyed. To reduce these risks, the computer room should not be located near external fire hazards, and combustible materials should not be stored nearby. Early warning fire detection equipment is necessary, and air circulation and ventilation fans should be connected to the fire detection system.

\subsubsection{Security}

Security depends on the building location, the surrounding areas, the interior layout, and the location of the computer room. Security precautions start at the street level and work toward the computer room. It should be zoned; e.g. separate entrances for employees and visitors, card-readers based on need-to-enter, alarm doors to limit passage. Sound, motion, and heat sensing devices as well as close circuit television systems are all useful in monitoring access to sensitive areas.

Entry to the room is monitored by a guard, a code operated electric door, or both. Placing the room in an enclosed inconspicuous location provides additional protection. 
Once admitted to a computer room, access to specific memory tapes, disks, control terminals and operating consoles may require added identification.

A central security console should be employed to monitor all systems and components. The console should be the termination point for all protection and monitoring systems and devices. Trained personnel should be employed for this function.

\subsubsection{Raised Access Floors}

This is the most convenient method of ensuring that equipment and systems can be readily updated and moved with minimum disruption to normal work activities. It provides ready access to ventilation, communications, power, and other building systems. Raised floors are subdivided into portable panels, permitting the exchange of plain panels with those with ventilation registers or cable exits.

The loading rating of the should be between 100-150 lbs per sq ft; with floors for UPS and backup power requiring more capacity. The walls should have 2 hour fire resistant ratings. Access floors should have at least 12 inches of clearance to house all of the necessary cabling; with 18 inches preferred if possible.

\subsubsection{Lighting}

The recommended approach is a combination of task lighting and indirect or parabolic lens ceiling lighting at a reduced level. Adjustable swivel arm task lighting is especially appropriate because it facilitates personal control. 
Developing appropriate designs for new office technologies poses a major challenge to the design and engineering professions. since office automation is a relatively recent phenomenon, and not yet subject to systematic study, it is premature to formulate performance criteria for office settings. However, designers have coped with new technologies for more than a decade using a variety of design practices. These practices, together with published material in design and research publications, form the basis for this report. Since developments in this fjeld have been rapid, a mechanism should be developed to acquire current information - perhaps by means of conferences or workshops, jointly sponsored by federal agencies and the private sector.

Office automation has profoundly affected the office as a place to work in ways not well understood. General agreement exists among office automation experts however that a diversity of interdependent issues merit consideration when planning to automate office functions. These issues include: management, information processing, human resources (ergonomics), telecommunications, technology, and design.

The differences between traditional and automated offices has increased the importance of office design as a contributing factor to organizational effectiveness. In traditional offices considerable opportunity exists for personal interaction among employees. For example, since most information is in paper form, it must be handled and transmitted manually from person to person. Exchanging information and report writing therefore provides the opportunity for conversation and social exchanges. There is also a one-on-one relationship between the person initiating a document and the one responsible for putting it in final form, e.g.typing it.

With the advent of electronic based information systems, a person at a workstation often communicates only with a VDT-based terminal during an eight hour workday. Little need exists in many activities to work personally with office colleagues. As a result, office workers have complained about a sense of isolation, and being subservient to technology (1). Office design can be used to alleviate these problems by providing places for informal conversations and a general appearance which provides visual relief from technology.

Technology has influenced the composition of the office workforce. More professionals and managers are in the workforce, as a greater percentage of people engage in white collar work. These knowledge workers want a "high quality" work setting, responsive to the mental and physical demands of their jobs. Questionnaire surveys have identified a desire for visual and aucitory privacy, glare-free lightjing, and control of the immediate environment as important design issues for knowledge workers (4). 
Unfortunately, as the quality of the work environment has become nore important, introducing technologies into offices has frequently resulted in deteriorated environmental quality, e.g. machines producing noise and heat placed on desks designeci to support paper-based activities. This has occurred because office automation has been largely hardware-driven, i.e. machines and systems bejng purchased because they purportedly increase office productivity. All too often insufficient planning typifies the automation of office functions, especially with respect to design consequences (9).

Looking toward the development of design criteria for office autornation, improved information is needed in the following areas:

* Determining the effectiveness of design practices used to date. Consiảerable experjence has been gained by the federal government aná the private sector, but few systematic findings are available in the public domain. Evaluations of approaches that "work" or don't "work" would result in considerable dollar savings.

* In recent years, office design has employed open-space planning for traditional anà automated office functions. Yet, surveys of office workers indicate many problems with such designs. Post occupancy evaluatjons of these designs should be conducted.

* Technological advancements have permitted the automation of many building systems, operational, service and environmental. However, tradeoffs associated with automation are not well understood and require investigation. For example, studies have shown that workers want more, not less control of their workspaces and environments (5).

* The integration of operational systems with other building service systems is receiving considerable attention by designers, but little consensus exists as to how to achieve this result. Field studies are required to develop the data needed for ciesign criteria to accomplish this important function.

* In response to the need for better office automation planning, an improved architectural programming process is needed. It should facilitate the incorporation of technologies into offices while minimizing the disruption of ongoing activities, and do so in a cost effective manner. 
1. Becker, F. Workspace: Creating Environments in Organizations,

Praeger, Pub., N.Y., 1981 .

2. Downs, A. "The Future of Office Buildings", Buildings,

September, 1981 .

3. Harris, L. \& Assoc. "The Steelcase Study of Office

Environments: Do They Work?", Steelcase, Grand Rapids, Mich., 1979 .

4. "The Office Building as a Tool to Increase Productivity and the Quality of Working Lifen, BOSTI, Buffalo, N.Y., 1982.

5. Quinan, M., et. at., "FSA Systems Furniture Evaluation - Final Report", BOSTI, Buffalo, N.Y., May, 1982.

6. Martin, A. "The Human Connection: A Strategy for Making Automation Work", Admin. Mgmt., Feb., 1982 .

7. Zuboff, S. "Psychological and Organizational Implications of Computer-Mediated Work", CISR \#71, MIT, Cambridge, Mass., June, 1981 .

8. Stout, E. "The Human Factor in Productivity: The Next Frontier in the Office", Journal of Micrographics, April, 1981. 9. Driscoll, J. "Office Automation: The Dynamics of a Technological Boondogglen in Emerging office Systems, ed. R. Landau, J. Bair, and J. Siegman, Ablex Pub. Co., Northwood, N.J., 1982 .

10. Damodaran, L. "Human Choices in the Office of the Future", IERE Conf. Proc. \#45, London, England, April, 1980. Il. Brown, B., Dismukes, K. and Rinalducci, E. "Video Display Terminals and Vision of Workers", Behaviour and Information Technology, Vol. 1, No. 2, 1982.

12. Rubin, A. and Murray, J. "Office Design and Implementation:

Case Studies", Unpublished Trip Report, Feb., 1983.

13. Hendricks, D., et. al. "Human Engineering Guidelines for Management Information Systems", Human Engineering Laboratory, Aberdeen, Md., Nov., 1982.

14. Kaplan, A. "The Ergonomics of Office Automation", Modern Office Procedures, May, 1982.

15. Marans, R. and Spreckelmeyer, K. "Evaluating Open and Conventional Office Design", Environment and Behavior, Vol. 14, No. 3, May, 1982 .

16. Veneklasen, $W$. "The Role of Habitability Information in PostOccupancy Evaluation", CERL Interim Report E-46, March, 1979. 17. Van Cott, H. and Kinkade, R. Human Engineering Guide to Equipment Design, U. S. Gov't Printing Office, Wash., D.C., 1972. 18. Video Displays, Work and Vision, National Academy Press, Washington, D.C., 1983.

19. Kroemer, R., and Price, D. "Ergonomics in the Office:

Comfortable Workstations Allow Maximum Productivity", Ind. Eng., July, 1982 .

20. Galitz, W., Human Factors in Office Automation, Life Office Mgmt. Assn., Atlanta, Ga., 1980.

21. Walker, $K$. "Open-Plan Acoustics: Part $I^{n}$, The Construction Specifier, Vol. 32, No. 2, Feb., 1979. 
22. Rubin, A. and Elder, J. Building for People, NBS Special Publication 474, U.S. Gov't Printing Office, Wash., D.C., June 1980 .

23. Winzer, G., Unpublished paper, Office Automation Design Roundtable, March, 1984 .

24. Hirtle, P. and Powers, N. "The OPLAN system: Interactive Computer Program for Acoustical Design of Open Plan Offices", Third Int'1. Symposium on the Use of Computers for Environmental Engineering in Buildings, Banff, Canada, 1978.

25. Hedge, A. "The Open-Plan Office: A systematic Investigation of Employee Reactions to their Work Environment", Environment and Behavior, Vol. 14, No. 3, May, 1982.

26. IES Lighting Handbook - Reference Volume, Illuminating Engineering Society, New York, 1981.

27. "Air Force Interior Design Pamphlet", AF Pamphlet 88-41, Dep't of the Air Force, Washington, D.C., October, 1980. 28. Wotton, E. "Lighting for Education", Canadian Ministry of Education, Ottawa, Ontario, Canada (Undated). 29. Shemitz, S. "Evaluating the Quality of Task/Ambient Lighting", Lighting Design and Applications, Vol. 9, No. 1, Jan., 1979 .

30. Hooker, M. Unpublished paper, Office Automation Design Roundtable, March, 1984 .

31. Murray, W. et. al. "Potential Health Hazards of Video Display Terminals", NIOSH Research Report 81-129, June, 1981.

32. Fanger, P.O. Thermal Comfort, Danish Technical Press, Copenhagen, Denmark, 1970.

33. Khosla, N. Unpublished paper, Office Automation Design Roundtable, March, 1984.

34. ASHRAE Handbook -Fundamentals, ASHRAE, New York, 1977. 35. Spengler, J. and Sexton, K. "Indoor Air Pollution: A Public Health Perspective", Science, Vol. 221, No. 4605, l July, 1983. 36. Hadley, J."Energy Conservation and Indoor Air Quality", ASHRAE Journal, Mar 1981.

37. Woods, J. "Office Pollutants: They Can be Controlled", The Office, March 1985.

38. McNall, P."Indoor Air Quality Status Report", CLIMA 2000, Copenhagen Press, 1985.

39. Caruba, A. "An Air Quality Time Bomb is Ticking", Modern Office Technology, April 1984.

40. Sterling, D., Sterling T.and McIntyre, D. "New Health Hazards in Sealed Buildings", AIA Journal, Apr 1983.

4l. Fisk, W. "Ventilation for Control of Indoor Air Quality", CLIMA 2000, Copenhagen Press, 1985.

42. "Wiring Systems: An Innovative and Maturing Technology", Arch. Record, Oct., 1982.

43. Cozette, C."The UPS and Downs of Power Protection", Infosystems, March 1985.

44. Bowers, D. "Understanding Todays Power Line Control Systems", The Office, May 1985.

45. Rosch, W. "Power Protection", PC Week, May 14, 1985. 46. Lazar, I. "Suppressing Overvoltage Transients", Electrical Construction and Maintenance, June 1980. 
47. Kimball, J. "Interest in power protection surges as supply of clean electricity sags", Data Communications, Mar 1985.

48. Rosch, W. "Backup Power Devices", PC Week, Nov 5, 1985.

49. McCraty, R. "Don't Put up with any Static!", Modern Office Technology, Feb., 1984 .

50. Sniger, P. "UPS Exhibit Increased Flexibility and Diversity", Mini-Micro Systems, April 1984.

51. Medina, S. and Helms, S. "Designing Intelligence into Buildings", Data Communications, Sept 1985.

52. Baker, $J$. "The Importance of Wiring to the Electronic Office", The office, Nov. 1985.

53. Wirl, C. "What's the best way to wire a new building for data?", Data Communications, Sept. 1985.

54. "Plugging into the Future", The Construction Specifier, Aug

55. "Selecting Access Flooring Systems for Offices", The

Construction Specifier, Aug 1984 .

56. Pearce, P. "Streamlining Interiors with Flat Conductor

Cable", Constr. Specifier, Nov, 1982.

57. Licklider, J. and Vezza, A. "Applications of Information Networks", Proceedings of the IEEE, Vol. 66, No. 11, Nov., 1978. 58. "PC Communications get in on the OSI Act" Systems and

Software, May 1985.

59. Dudley, R. "Checklist of Key Questions to Ask When Buying Automated Telecom Accessories", Comm. News, Dec., 1982.

60. Geisler, C. "Some Ideas to Consider in Seeking a Local-Area Network" The Office, Feb., 1984.

61. Siragusa, G. "A Manager's Guide to Office Wiring Systems",

Administrative Management, Jan. 1986.

62. Shoch, J. et. al. "Evolution of Ethernet Local Computer

Network", Xerox, Palo Alto, Cal., Sept., 1981.

63. Potts, J. "LANs- Where We Stand Today", Government Data Systems, Nov/Dec 1984 .

64. Ginsburg, L. and Rappaport, D. "Half Empty or Half Full", Datamation, March 1986.

65. A. D. Little Table (Correspondence)

66. King, J. "Local Area Networks: More Smoke than Fire"?,

Computerworld, Oct $3,1984$.

67. Stallings, W. "Beyond Local Networks", Datamation, Aug 1983

68. Cooper, E. "Local Area Network Installation", Computerworld Focus.

69. Scott, T. "How to Avoid Some Common Pitfalls of a Local Net Installation" Data Communications, Mar 1984.

70. Ruby, D. "Local Area Network Software", PC Week, Feb 12 , 1985 .

71. Geisler, C. "Some Ideas to Consider in Seeking a Local Area Network, The Office, Feb. 1984.

72. Yaski, E. "Is there a PBX in your future"?, Datamation, Mar 1983.

73. Goeller, L. and Goldstone, J. "The ABCs of the PBX", Datamation, April 1983.

74. Dell, D. "PBX: The Switch is On", MIS Week, May 9, 1984.

75. Rice, S. "The Great Data-Switching Fallacy", MIS Week, Sep 5, 1984. 
76. Angus, I. "Networking Micros on the Digital PBX", Computerworld, Oct 3, 1983 .

77. Paznik, J. "Digital PBX- A Networking Alternative", Admin Mgmt, Jan 1986.

78. Wecker, S. "Combining Voice and Data Through Local Nets and PBX" , Computerworld Focus, September 1984.

79. Meade, P. "Centrex and PBX's in a Tough Fight",

Communications Week, Sept 2, 1985 .

80. GSA/PBS memorandum on planning High Technology Office

Builaings.

81. "The Integrated Building: Greater than the Sum of its Parts", Teleconnect, August 1985 .

82. LeBlanc, $R$. "Controlling the Intelligent Building",

Conference Paper (Personal Communication).

83. Carlini, J. "Intelligent Buildings", Government Data Systems, May 1986.

84. Schell, T. "Developing Communication", Buildings, Aug 1983. 85. Hasty, S. "Intelligent Buildings Come of Age", National Real Estate Investor.

86. Solomon, S. "Compatibility at Citiplace", Management Technology, February 1984.

87. King, J. "Communications Options Rise with Intelligent

Buildings", Information System News, Nov. 26. 1984.

88. Cross, T. "Smart Buildings Next on the Horizon", Management Information Systems Week, April 4, 1984.

89. Valentine, P. Patri, P. "Smart Building Checklist", (Personal correspondence) sept. 1985.

90. Viladas, P. "Interior Technics: Open-Office Partition

Systems", Prog. Arch., May, 1982.

91. Rubin, A. "The Automated Office - An Environment for

Productive Work, or an Information Factory?: A Report on the State-of-Art", NBSIR 83-2784-1, National Bureau of Standards, Nov., 1983.

92. Gold, E. "The Importance of Design in a Teleconference Room", The Office, Nov., 1982.

93. Terjesen, J. "A Computer Room for Machines and People", Modern Office Technology, March 1985.

94. Bassenhoffer, R. "Design and Site Selection: Key to Avoiding Problems", Govt Computer News, Nov. 8, 1985.

95. Engleberg, W. "Computing's Critical Environment", Govt Data Systems, Nov/Dec 1983.

96. Symmes, S. "Designing a Data Center", Modern Office

Technology, Dec. 1983.

97. "Guideline on Electrical Power for ADP Installations", EIPS Publication 94, National Bureau of Standards, September 1983. 
ACCESS TIME- The time interval between data request and delivery. ACOUSTIC COUPLER- A modem enabling a remote device to be connected to devices or data bases using a conventional telephone, dialed over a leased public line.

AMBIENT LIGHTING- Lighting throughout an area that produces general illumination.

ANALOG COMPUTER- A calculating machine that operates with numbers represented by directly measurable quantities (as voltages, resistances, or rotations).

ANTHROPOMETRY - The study of human body measurements, especially on a comparative basis.

AI- Articulation Index

ARPANET- A set of autonomous, interconnected, and independent computer centers which use a standard transmission method to permit interactive resource sharing among all participants--set up by ARPA (Advanced Research Project Agency). ARTICULATION INDEX- A numerically calculated measure of the intelligibility of transmitted or processed speech. It takes into account the limitation of the transmission path and the background noise.

ASCII- American Standard Code for Information Exchange. A coding system for characters of seven bits plus one parity bit, for transmission over a dara network.

ASHRAE- American Society of Heating, Refrigerating, and Air Conditioning Engineers, Inc.

ASYNCHRONOUS- A transmission sequence where the intervals between characters are not equal; start and stop bits are added for coordination.

ASYNCHRONOUS MODEM- A communications channel capable of transmitting data but not able to time the information. AUXILIARY STORAGE- Any peripheral device where data may be stored, e.g. disk, tape.

BASEBAND - The band of frequencies occupied by all transmitted signals used to modulate the radio wave produced by the transmitter in the absence of a signal.

BATCH PROCESSING- A technique that uses a single program loading to process many individual jobs, tasks or signals for service. BAUD- A measure of the transmission speed capability of a communications line or system. In a sequence of binary signals, 1 baud $=1 \mathrm{bit} / \mathrm{sec}$.

BINARY CODE- A coding system employing the binary digits one and zero to represent letters, digits, or other characters in a computer.

BIT- The smallest entity in the memory of a computer

BITS PER SECOND (BPS) - A measure of data transmission speed, equal to the number of changes per second.

BPS- Bits per second.

BRIDGE- Circuitry used to connect networks with a common set of high-level protocols.

BROADBAND - A band with a wide range of frequencies. BUFFER- A small storage space, useả for temporary storage of data before processing. 
BUS- A length of coaxial cable, to which microcomputer stations are attached.

BYTE- A term used to describe the storage in a computer; it may contain 8,16 or 32 BITS, depending on the computer design. CENTRAL PROCESSING UNIT- The computer at the center of an on-line system which performs the processing according to the applications package.

CIS-Computer based information system.

CLUSTER- A data network in which two or more terminals are connected to a line or data channel at a single point. CMBS- Computer based information system. COAXIAL CABLE- A transmission line in which one conductor is contained inside and insulated from an outer metal tube that serves as a second conductor; used for networks and connecting terminals to mainframe computers.

COEFFICIENT OF UTILIZATION- The ratio of luminous flux (Iumens) from a luminaire calculated as received on the work-plane to the luminous flux emitted by the luminaire's lamp alone. CoM- Computer output microfilm

COMMAND LANGUAGE- A type of dialogue in which the user formulates control entries with minimal prompting by the computer. COMPUTER MAIL- The transmission of messages using computer systems. CPU - Central processing unit

CONTRAST RENDITION FACTOR- The ratio of visual task contrast with a given lighting environment to the contrast with sphere (uniform) lighting. CORE STORAGE- The memory or internal storage of a computer. CPU- Central Processing Unit.

CRITICAL SUCCESS FACTORS (CSF) - Those activities most important in performing major job functions.

CU- Coefficient of Utilization.

DATA BASE MANAGEMENT SYSTEM- General purpose programs that accept data in a format determined by the user, process, the data, the output it in a desired format.

dB- Decibel.

DBMS- Data base management system

DDP- Distributed data processing

DECIBEL- The unit of sound intensity.

DECISION SUPPORT SYSTEM- A computer information system designed

for managers and professionals, using simplified interactive

programming with access to one or more data bases.

DIALOGUE- A structured series of interchanges between a user and

a terminal. They can be computer-initiated (question and

answer) or user controlled by a command language.

DIFFUSER- A device to redirect or scatter the light from a

source, primarily by diffuse transmission.

DIGITAL SWITCH - Interconnecting data, word processing, and telephone equipment by a digital PBX.

DISABILITY GLARE- Glare resulting in reduced visual performance and visibility.

DISCOMFORT GLARE- Glare producing discomfort; not necessarily interfering with performance

DISTRIBUTED DATA PROCESSING- Sharing of computing among several computers at different locations. 
DP- Data processing

DSS- Decision support systems

DUPLEX- The ability to simultaneously transmit and receive signals over a network.

DYNAMIC DISPLAY - A display that changes automatically as a result of system action.

EDP- Electronic data processing.

EFFECTIVE TEMPERATURE- A combination of air temperature, air velocity, and relative humidity, used to define a thermal comfort zone for people in buildings.

ELECTRONIC MAIL- An electronic system using communications lines, computers, CRT and hard copy terminals to transmit documents and messages to individual addresses.

ERGONOMICS- A subject are which deals with the interaction of person/machine and person/environment issues.

ET- Effective Temperature.

ETHERNET- An baseband local network, with several microcomputers, based upon workstations performing a given function, with data

processing capability.

FACSIMILE- Analog or digital transmission of page images.

FDM- Frequency division multiplexing

FEEDBACK - A response from the system which informs the user of the status of current requests or commands.

FIBER OPTICS- Very thin strands of glass, used to transmit information by means of light pulses.

FILE- A collection of related records, usually arranged sequentially according to a directory key contained in each record.

FIRMWARE- Routines wired into the computer as part of the circuitry. FLANKING PATH- Noise transmission by an indirect path, e.g.

structural element.

FLOPPY DISC- A small mylar data storage device, resembling a

phonograph record.

FRONTEND SYSTEM- The part of the computer system that interprets what the user specifies, and interacts with the "background" of the system, which performs the detailed operations desired.

FULL DUPLEX- The simultaneous independent transmission of data in both directions over a communications line.

GATEWAY - System and software permitting networks using different

protocols to communicate with one another.

HABITABILITY- The impact of the environment on the behavior of

building users with respect to their welfare, task performance, and job satisfaction.

HALF-DUPLEX- Users can transmit data in one direction at a time, with each user signaling a completed transmission before the next one can begin.

HERTZ- Cycles per second

HID- High intensity discharge

HIGH INTENSITY DISCHARGE- A general group of lamps consisting of

mercury, metal halide, and high pressure sodium sources.

HOST COMPUTER- A system in a network performing data processing

operations and with which network nodes communicate.

IBD- Institute of Business Designers

IEEE- Institute of Electrical and Electronic Engineers

IES- Illuminating Engineering Society 
IEEE- Institute of Electrical and Electronic Engineers IES- Illuminating Engineering Society

IESNA- Illuminating Engineering Society of North America.

INTELLIGENT TERMINAL- Terminal with limited programming

capabilities such as format creation and parameter definition.

INTERFACE- An electronic device enabling one item of equipment to communicate with or control another one.

INVERTER- A device that converts low-voltage DC to high-voltage AC.

IS, I/S- Information system

Iso- International Standards Organization

JOYSTICK- Manual input device for graphic display consoles, which allows the user to specify 2 dimensional or 3 dimensional

coordinates.

KBITS- Kilobits

KHZ- Kilohertz

KILOBITS- Thousands of bits.

KNOWLEDGE WORKER- A person with a major responsibility for developing, analyzing and/or acquiring information, e.g., professional, manager.

LAMP LUMEN DEPRECIATION FACTOR- The multiplier used in lighting calculations to relate initial rated output of light sources to the anticipated minimum output based on the relamping program to be used.

LAN- Local area network

LOCAL AREA NETWORK - Systems of computers and peripheral devices linked together in adjacent offices or buildings.

LUMINANCE CONTRAST- The relationship between the luminance of an object and that of its immediate background.

MAINFRAME- The central processing unit of a large computer

system.

MANAGEMENT INFORMATION SYSTEM - A computerized procedure for providing managers with immediate knowledge, information, and data needed to make decisions, direct people, and regulate operations to better attain organizational goals.

MANUAL PROCESSING- Any data processing operation performed by hand.

MBITS- Megabits

MEGABITS- Millions of bits

MENU- A type of dialogue in which the user selects from a number of displayed alternatives.

META LANGUAGE- A set of symbols and words used to describe another language (in which these symbols do not appear).

MHZ- Megaherts

MICROCOMPUTER- A term describing the small physical size of a computer.

MICROGRAPHICS- The capture, retrieval, and display of miniaturized, high-resolution photographic images containing either textural or graphic information. MINICOMPUTER- A computer whose physical size is between a microcomputer and a full size computer. MIS- Management information system

MICROCOMPUTER- Used to described the small physical size of a computer. 
MODEM- A device the converts analog signals to digital ones and visa versa (Modulator/DEModulator).

MULTI-DROP-LINE- A circuit dedicated to communications among multiple terminals and peripherals on the same circuit.

MULTIPLEX - to interleave or simultaneously transmit two or more messages on a single channel.

NC CURVES- Noise Criteria Curves.

NODE- A single station in a local area network

NOISE CRITERIA CURVES- Criteria to rate the acceptability of continuous noise levels for performing given activities.

$O A$ - Office automation

OCR- Optical character reader

OFF-LINE SYSTEM- A system configuration in which the input/output devices, such as terminals, are not in direct communication with a central processor.

ON-LINE-SYSTEM- A systems configuration where input/output

devices, such as terminals are in direct communication with a

central processor.

OPEN SYSTEM INTERCONNECTION- A model developed by the

International standards Organization (OSI) to describe a network open to equipment from different manufacturers.

OPERATING SYSTEM- Software routines for interfacing activities of system components (e.g. disks, CRT's, printers), tailored to each device.

OPTICAL CHARACTER READER- The reading and identification of

characters optically, under computer control.

OSI- Open system interconnection

PABX - Private automatic branch exchange

PACKET SWITCHING- A transmission method in which data are assembled in small packets before being transmitted from node to node across the network.

PARITY - The condition of the number of items in a group, e.g. bits in a byte, being odd or even; to check for errors.

PARITY CHECK - The automatic transmission of additional parity

bits to enable the receiving device to check the accuracy of

transmitted data.

PBX - Private branch exchange

POINT-TO-POINT- Dedicated transmission between two devices.

POLARIZATION FILTER- A type of screen filter reducing the

reflection of the incident light on the screen.

POWER SURGE- An increase of voltage levels $10 \%$ or more above

normal, lasting from one half cycle to several seconds.

PROTOCOL- A set of rules governing the format, timing and error control of messages, to ensure compatibility of data transmitted over a network.

PROTOCOL CONVERTER- Enables ASCII terminals and microprocessors to function in various mainframe and other environments.

QWL- Quality of working life

RAM - Random access memory

RANDOM ACCESS MEMORY- Solid state machine memory, independent from external storage.

READ ONLY MEMORY - Routines and languages wired into a plug-in circuit card. 
REFLECTED GLARE- Glare from specular reflections of high luminances on polished or glossy surfaces in the field of view. RF- Radio frequency.

RH- Relative humidity.

RING- A network topology in which stations are arranged in a circle.

ROM- Read only memory

RS-232-C- An electrical standard used for serial ports.

SCIENTIFIC MANAGEMENT- Analysis of tasks into smallest possible standard components; "time-and-motion" studies to increase productivity.

SEMANTICS- The meaning of language intended by the originator of a message.

SERIAL DATA TRANSMISSION- A method of data transmission whereby characters or bytes are sent one bit at a time over a single path.

SERIAL PORT- An input/output port that transmits data one bit at a time.

SIGNAL TO NOISE RATIO- The ratio of signal intensity to noise intensity, usually expressed as the decibel difference between the signal and noise levels.

SIL- Speech Interference Level.

SNA- Systems Network Architecture

SOFTWARE- Computer programs, rules, procedures, and associated documentation concerned with the operation of a computer-based system.

SOUND ABSORPTION - The change of sound energy into another form, usually heat, in passing through a medium or striking a surface. SOUND TRANSMISSION CLASS- The preferred single figure rating scheme giving an estimate of the sound insulation properties of a partition. Used when speech and office noise constitutes a major problem.

SOUND TRANSMISSION LOSS- A measure of sound insulation. The amount of incident sound passing through a partition.

SPEECH INTERFERENCE LEVEL- The arithmetic average of the sound pressure levels of noise in the three octave bands centered at 500,1000 , and 2000 herz.

SPIKE- A sudden but brief electrical disturbance, reaching thousands of volts, with frequencies up to the megaherz level. STAR- A network topology in which terminals are connected to a central processor in a spiked configurations, like points in a star.

STC- Sound transmission class

SYNCHRONOUS- A transmission sequence in which characters are packaged in blocks, defined at the beginning and the end of the transmission by codes.

SYSTEMS NETWORK ARCHITECTURE (SNA) - A communications model developed by IBM, that integrates computer systems and other devices.

SYNTAX-A set of rules specifying which forms of the language are grammatically acceptable.

TASR/AMBIENT LIGHTING- A combination of task and ambient lighting in a given area, with the ambient lighting being lower in

intensity and complimentary to the task lighting. 
TASK ANALYSIS- Used to determine the psychological and physical factors essential to the adequate performance of a task. TDM- Time division multiplex

TERRITORIALITY - The perception of a person or a group that they possess a given place to the exclusion of others.

TIMESHARING- A function of software that enables parceling out access to the processor among input/output devices such as VDT's often by means of dial-up ports.

TOKEN- A software message that circulates among network nodes. TREE- A network architecture in which stations of equal status are aligned along a bus cable.

TRUNK- A specialized circuit used to connect circuit switches to one another.

TWISTED PAIR- A cable comprised of two small insulated conductors twisted together without a common covering.

TWX - A network of telegraph-grade communications lines and terminals used to transmit messages.

UPS- Uninterruptable power systems.

USABILITY - The ease of learning a task, and ease of use. USER FRIENDLY - Requiring only a limited amount of experience, knowledge or training to operate.

VALUE-ADDED NETWORK- Transmission networks used to facilitate communication among terminals and computers.

VCP- Visual Comfort Probability

VDT- Video display terminal

VDU- Visual display unit

VEILING REFLECTIONS- Regular reflections superimposed upon

diffuse reflections from an object that partially or totally

obscures the details to be seen, by reducing the contrast. VISUAL COMFORT PROBABILITY- The rating of a lighting system expressed as a percent of people who, when viewing from a specified location and in a specified direction, will be expected to find it to be acceptable in terms of discomfort glare.

WHITE NOISE- An acoustical stimulus composed of all audible

frequencies at the same intensity with random phase relations between them; it sounds like "shhhhh".

WIDEBAND- A transmission medium capable of passing more frequencies than the standard $3 \mathrm{Khz}$ voice channel.

WORD PROCESSING- The keyboarding, editing, and printing of documents on a system that includes hardware and software in many instances.

WORKSTATION- The general physical environment in which the user works. It includes computer terminals, source documents, desks, chairs, and lighting.

WP- Word processing 

BIBLIOGRAPHIC DATA

NBSIR $86-3430$

4. TITLE AND SUBTITLE

Revised Interim Design Guidelines for Automated Offices

5. $A \cup T H O R(S)$

Arthur I. Rubin

6. PERFORMING ORGANIZATION (If joint or other than NBS, see instructions)

7. Contract/Grant No.

NATIONAL BUREAU OF STANDARDS

DEPARTMENT OF COMMERCE

WASHINGTON, D.C. 20234

8. Type of Report \& Period Covered

NBSIR 3/85- Present

9. SPONSORING ORGANIZATION NAME AND COMPLETE ADDRESS (Street, City, State, ZIP)

General Services Administration

18 th \& 19th \& F. Sts. N.W.

Washington, D.C. 20405

10. SUPPLEMENTARY NOTES

Document describes a computer program; SF-185, FIPS Software Summary, is attached.

11. ABSTRACT (A 200-word or less factual summary of most significant information. If document includes a significant bibliography or literoture survey, mention it here)

This report is an update of an earlier design guideline (NBSIR 84-2908). It is based upon an additional literature search and interviews. The information should still be considered tentative, since they are still based on judgement and practice, not formal studies.

The introduction of automation into offices has changed the office as a workplace. Architects and other design professionals have responded to this technology by employing a number of design strategies. This report identifies design issues which merit consideration by the designer and suggests criteria and approaches that might be used in automated office design. Technological, ergonomic and organizational factors are all considered from the standpoint of their design implications. The present document is a major revision of the earlier study, including more than twice the number of reference documents than its predecessor.

12. KEY WORDS (Six to twelve entries; alphabetical order; capitalize only proper names; and separate key words by semicolons) Acoustics; design criteria; electrical systems; ergonomics; lighting; noise; office automation; office design; workstations; communication systems.

\section{AVAILABILITY}

[X] Unlimited

$\square$ For Official Distribution. Do Not Release to NTIS

$\square$ Order From Superintendent of Documents, U.S. Government Printing Office, Washington, D.C. 20402.

[X] Order From National Technical Information Service (NTIS), Springfield, VA. 2216I
14. NO. OF PRINTED PAGES

178

15. Price

$\$ 16.95$ 


